\title{
Using a Multi-Omic Approach to Investigate a Diet Intervention in Young Adults at Risk of Disease
}

Oluremi Ariel Famodu

Follow this and additional works at: https://researchrepository.wvu.edu/etd

\section{Recommended Citation}

Famodu, Oluremi Ariel, "Using a Multi-Omic Approach to Investigate a Diet Intervention in Young Adults at Risk of Disease" (2017). Graduate Theses, Dissertations, and Problem Reports. 7083.

https://researchrepository.wvu.edu/etd/7083

This Dissertation is protected by copyright and/or related rights. It has been brought to you by the The Research Repository @ WVU with permission from the rights-holder(s). You are free to use this Dissertation in any way that is permitted by the copyright and related rights legislation that applies to your use. For other uses you must obtain permission from the rights-holder(s) directly, unless additional rights are indicated by a Creative Commons license in the record and/ or on the work itself. This Dissertation has been accepted for inclusion in WVU Graduate Theses, Dissertations, and Problem Reports collection by an authorized administrator of The Research Repository @ WVU.

For more information, please contact researchrepository@mail.wvu.edu. 


\title{
Using a Multi-Omic Approach to Investigate a Diet Intervention in Young Adults at Risk of Disease
}

\author{
Oluremi Ariel Famodu
}

\author{
Dissertation submitted \\ to the Davis College of Agriculture, Natural Resources, and Design \\ at West Virginia University
}

In Partial Fulfillment of the requirements for the degree of

Doctor of Philosophy in Animal and Food Science

\author{
Melissa D Olfert, DrPH, RDN, Chair \\ Janet Tou, Ph.D. \\ Chris Cuff, Ph.D. \\ Pamela J Murray, MD, MHP \\ Mark Olfert, Ph.D. \\ Ida Holásková, Ph.D.
}

Department of Human Nutrition and Foods

\author{
Morgantown, West Virginia \\ 2017
}

Keywords: Young Adults, Nutrition, Behaviors, Microbiome, Metabolome, Metagenomics 


\section{Abstract \\ Using a Multi-Omic Approach to Investigate a Diet Intervention in Young Adults at Risk of Disease}

\section{Oluremi Ariel Famodu}

Background: Poor dietary behaviors are widely recognized in the role of development and course of a variety of illnesses, including but not limited to cardiovascular disease, obesity, and diabetes. Young adults have increased risk due to poor dietary behaviors, especially as they transition into a college setting. The evidence demonstrates an urgency for developing treatment and prevention through dietary intervention approaches in this population. In addition to improper diet, investigators have connected abnormal gut microbiome (i.e. the collection of genes from our intestinal microbiota) to inflammation and obesity, therefore momentum has gained in treating the altered gut microbiome with dietary manipulation to decrease morbidity and mortality.

Furthermore, research is moving towards metagenomic work to investigate mechanisms in disease progression that link genes that contribute to the microbes and the host.

Objective: By using young adults "at-risk" of disease, the FRUVEDomics pilot study investigated the effects of a dietary intervention, based on the United States Department of Agriculture's (USDA) 2010 Dietary Guidelines for Americans, on nutritional behaviors, the metabolome, and the microbiome. Additionally, relationships between the three variables were explored.

Methods: This pilot study stemmed as a subproject off of a USDA, Agriculture and Food Research Initiative (AFRI) called 'Get Fruved', which ignited the collaboration of a team of scientist (nutrition, metabolomics, microbiome, cardiovascular, microcirculation and physiology) from West Virginia University (WVU) (Clinical Trials Record 1409433435). Thirty-six young adults were randomized into three diet plans; a FRUVED diet (50\% fruit and vegetable), a FRUVED and low simple carbohydrate diet (FRUVED+LRC), and a FRUVED and low-fat diet (FRUVED+LF). Biochemical, anthropometric, serum, and fecal stool samples were collected pre and post intervention, along with subjective behavioral measurements via survey administration. Results: The dietary intervention resulted in an adjusted dietary change for all individuals, which included increases in fruit and vegetables and total fiber, with simultaneous decreases in saturated fat and empty calories. No other lifestyle behavior modifications were seen during the course of the 8 weeks (i.e. sleep, physical activity and stress), therefore the decrease we observed in systolic blood pressure, waist circumference, and total cholesterol were due to the treatment of an overall healthy diet. Pro-inflammatory markers improved and decreases were observed in total ceramides after the intervention. Next generation sequencing of genomic DNA from fecal samples showed decreases in Firmicutes and increases in Bacteroidetes after dietary manipulation. At the family level, increases in S24-7 and Bacteroidaceae, and decreases in Ruminococcaceae were found. Relationships were found between dietary variables, serum amino acids, and bacteria.

Conclusion: The researchers of the FRUVEDomics study observed improvements in metabolic health and changes to microbial populations that have been linked to energy balance and lowered inflammation after adoption of a healthy diet among young adults attending a university. Overall, this study lays the foundation for personalized medicine among a college population. In particular, this translational, team science approach helped determine mechanisms by which microbes affect production of metabolites from diet. 


\section{Acknowledgements}

I would like to express my sincerest gratitude to my advisor, Dr. Melissa Olfert, for her guidance, mentorship, encouragement, and for providing me with endless opportunities such as the FRUVEDomics project. I admire her creativity and passion for translational and communitybased participatory research. Her expertise and wisdom has inspired me to move my dreams and professional goals forward.

I would also like to thank my committee members Dr. Janet Tou, Dr. Ida Holásková, Dr. Pamela J Murray, Dr. Christopher Cuff, and Dr. Mark Olfert for each time they spent helping me to develop my dissertation and the constructive suggestions during planning and development of the project. I am blessed to have worked with such amazing researchers. My appreciation also extends to old and current members of the Lifestyle Intervention Lab, in particular Makenzie Barr and Jade White, for their help in various aspects of this project and other professional engagements.

Finally, I owe my deepest gratitude to my friends and family for their unconditional support and encouragement throughout these three years. I love each of you tremendously. 


\section{Table of Contents}

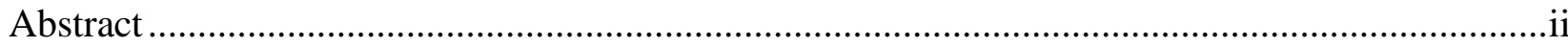

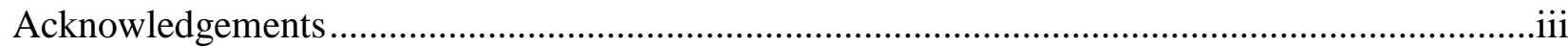

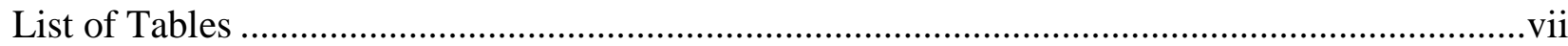

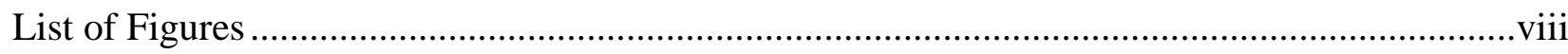

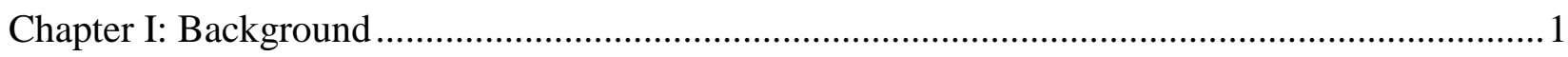

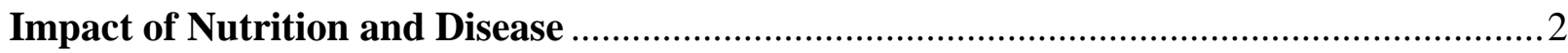

Dietary Behaviors among College Students and Appropriate Interventions ........................ 4

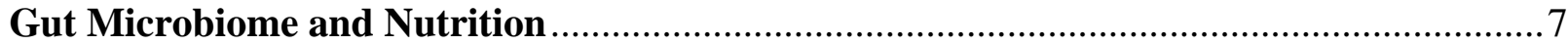

Metagenomics, Translational Research, and Team Science .............................................. 10

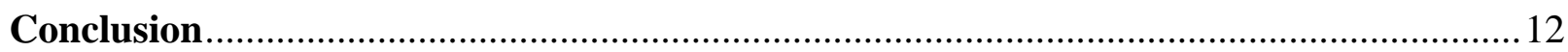

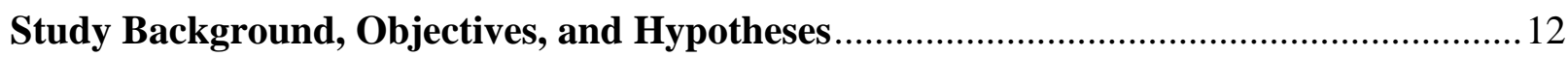

Chapter II: Efficacy of a Personalized Nutritional Intervention on Food Consumption and Metabolic Parameters in Young Adults ........................................................................................ 14

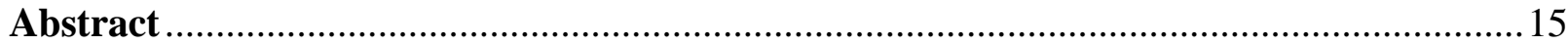

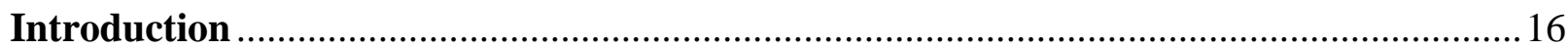

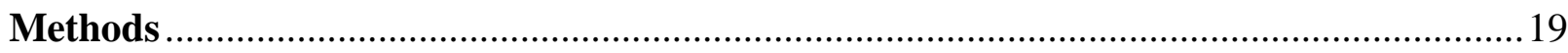

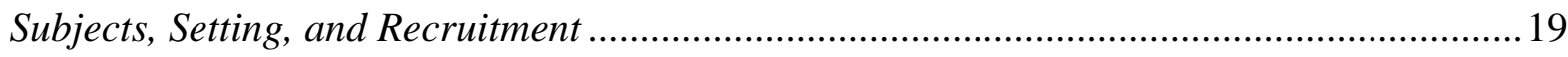

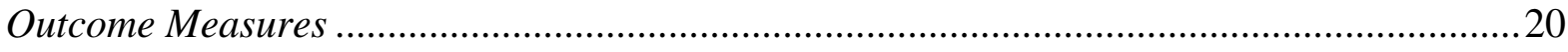

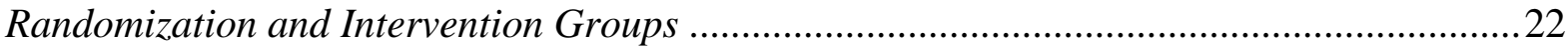

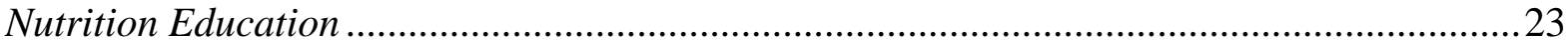

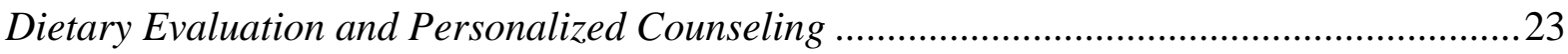

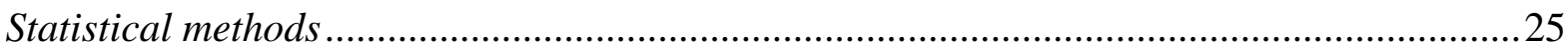

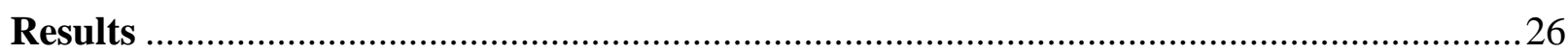

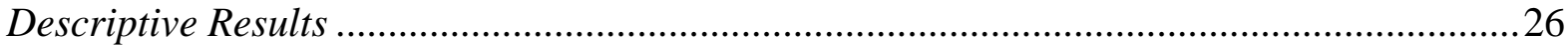

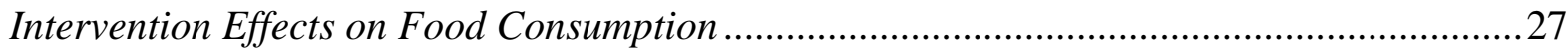

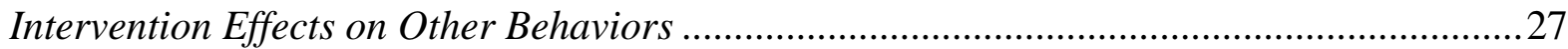

Intervention Effects on Metabolic Parameters .................................................................. 28

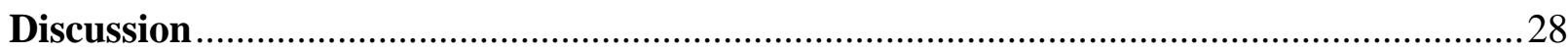

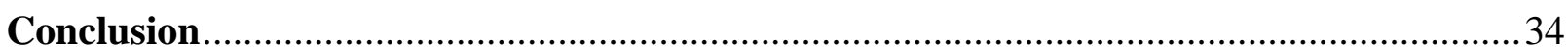

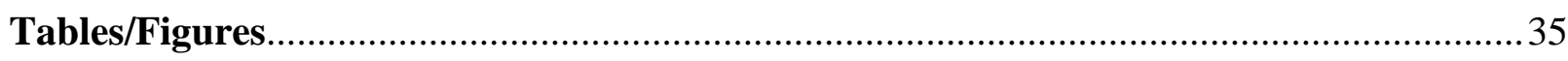

Chapter III: Impact of a Nutrition Intervention on the Microbiome of Young Adults ....................46

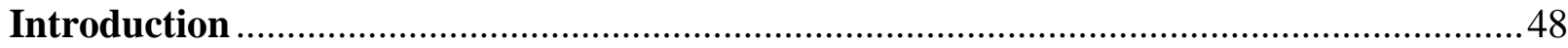




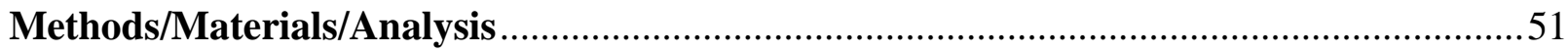

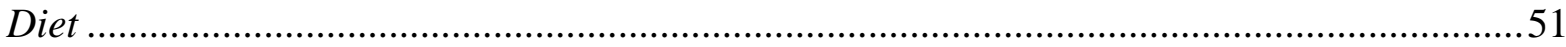

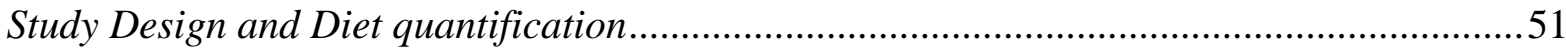

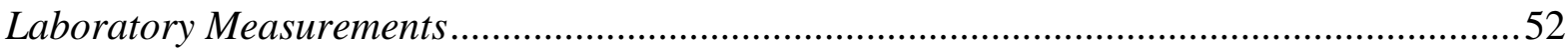

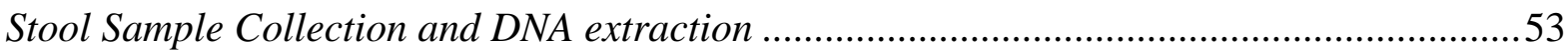

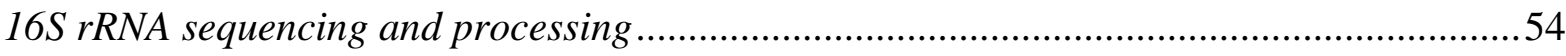

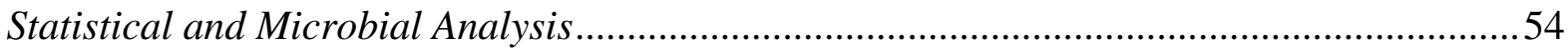

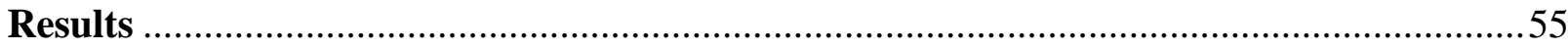

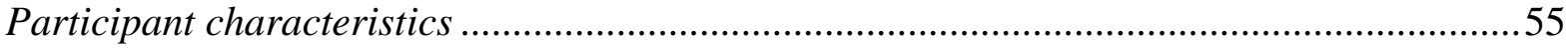

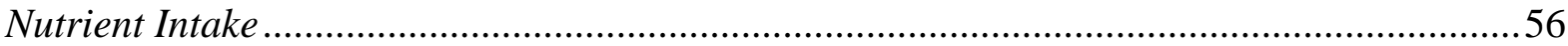

Anthropometrics, Biochemical and Amino Acid Metabolites................................................57

Fecal Microbiota Composition and Changes....................................................................5

Microbial Metagenomics ....................................................................................................58

Microbiome Interactions with Diet and Amino Acids ........................................................58

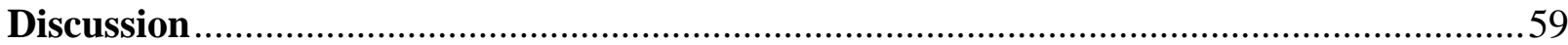

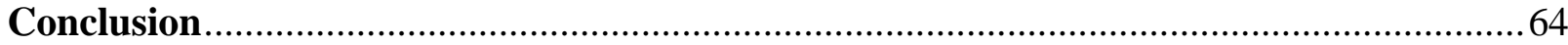

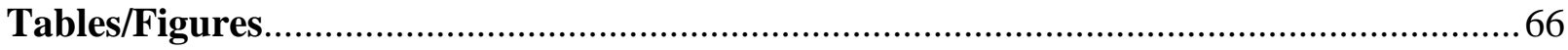

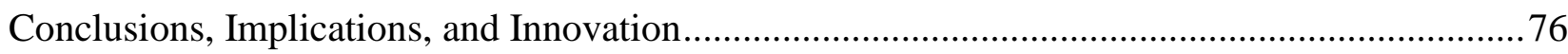

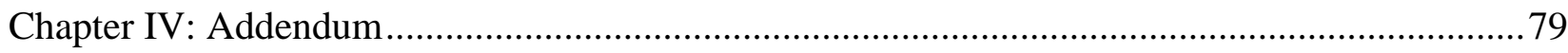

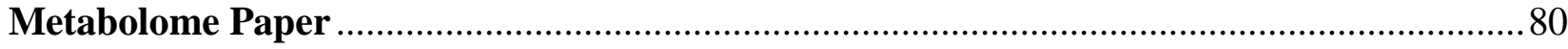

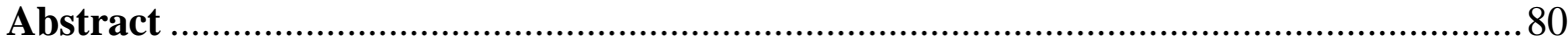

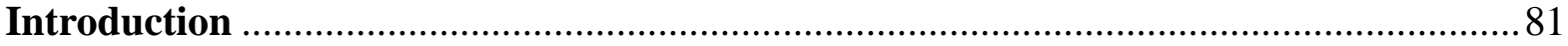

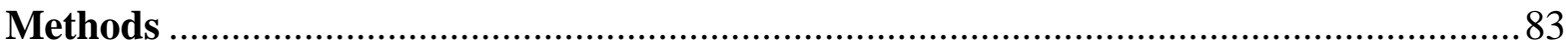

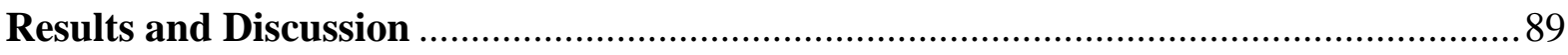

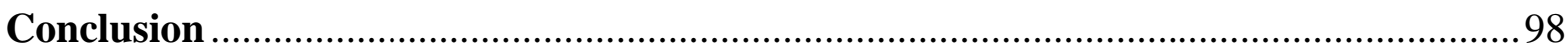

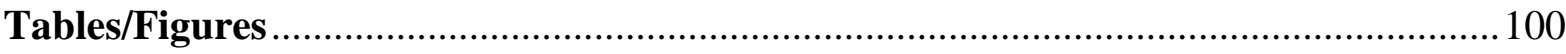

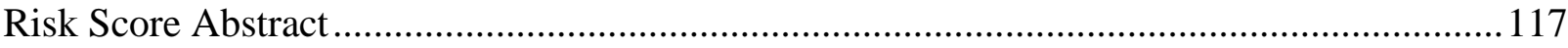

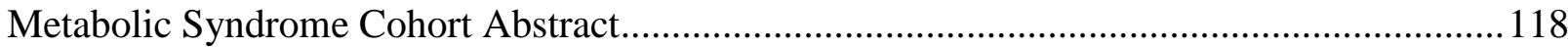

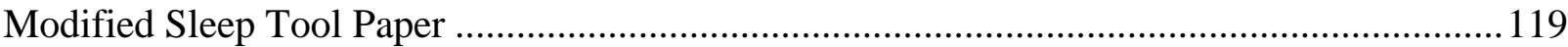

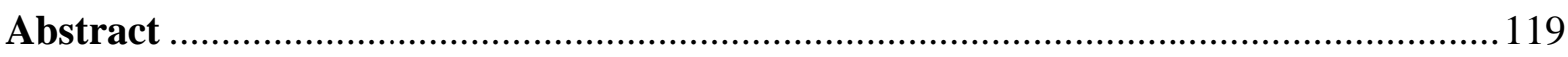

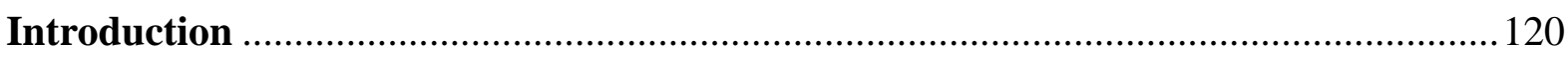

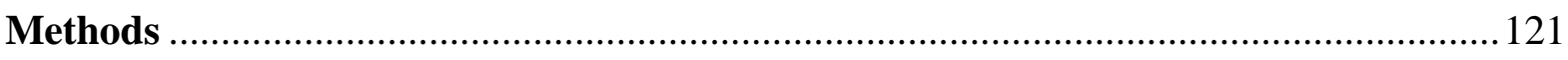

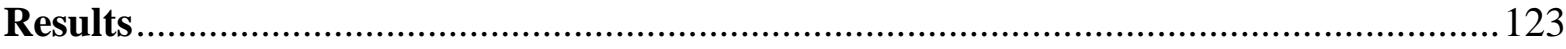




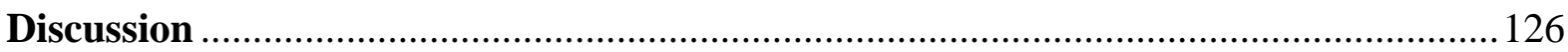

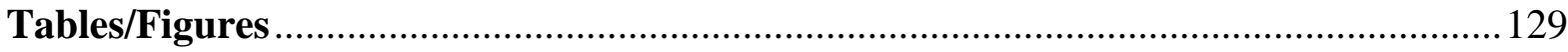

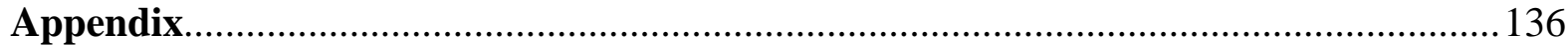

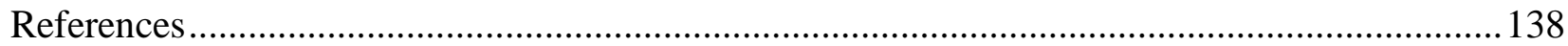

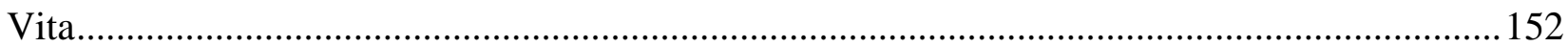




\section{List of Tables}

Name

Page

Dietary Arms in FRUVEDomics Study. $.35 \& 66$

Demographic and Health Related Characteristics at Baseline ...... $.36 \& 67$

Treatment Effects on Dietary Factors across the Duration of the Study...................................37

Treatment Effects on Physical Activity, Stress and Sleep on Compliant Individuals.....................38

Treatment Effects on Clinical Measures Pre \& Post Intervention on Compliant Individuals........39

Treatment Effects on Amino Acids from pre to post intervention on Compliant Individuals ......68

Multiple regression on phyla with dietary and serum amino acids at baseline...............................69

Multiple regression on phyla with dietary variables and serum amino acids post intervention......70

Demographics and metabolic syndrome risk score for compliant intervention subjects...............100

Anthropometric and blood pressure measurements before and after intervention...................... 101

Serum metabolic health measurements before and after intervention........................................102

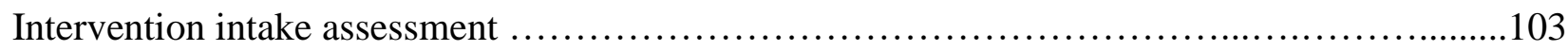

Serum cytokine measurements before and after intervention...........................................104

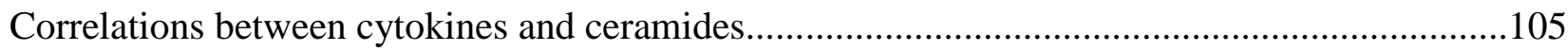

Items of the 19-Item (original) and 12-item ("shortPSQI") survey...........................................129

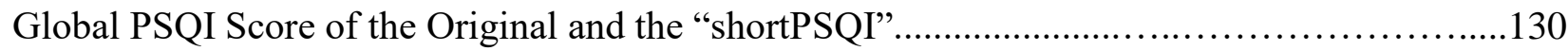

Agreements Tests between "Good" and "Bad" Sleeper Scored by Original and "shortPSQI"....131

Specificity and Sensitivity of Different Cut-Off values for "Good" and "Bad" Sleeper Scored by

Original and "shortPSQI" 


\section{List of Figures}

Name Page

Nutritionist Pro $^{\mathrm{TM}}$ analysis document used for individual counseling........................................40

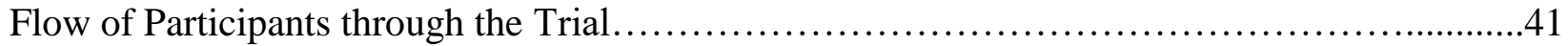

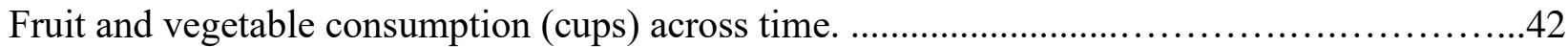

Empty calories (calories from solid fat and added sugars) consumption across time.....................43

Insoluble fiber consumption (grams) across time........................................................................

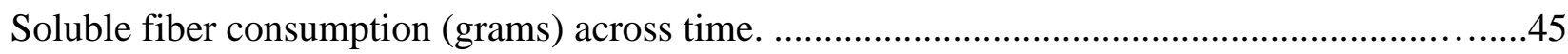

Microbiota composition of the study population after Baseline and Post Intervention for Phylum

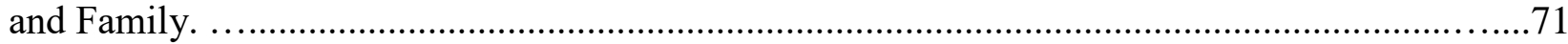

Microbiome changes in phylum and family taxa using inverted beta binomial paired testing......72

Bacterial metagenomes predicted from microbial community identities........................................73

Baseline significant stepwise regressions $(b=$ slope) among taxon relative abundance, dietary

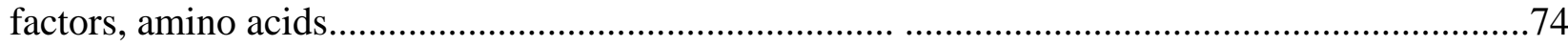

Post intervention significant stepwise regressions $(\mathrm{b}=$ slope $)$ among taxon relative abundance, dietary factors, amino acids factors...............................................................................................75

Total (A) ceramide, (B) GlcCer, and (C) LacCer concentrations in FRUVED (50\% fruit and vegetable), FRUVED + LRC (FRUVED plus low refined carbohydrate), and FRUVED + LF (FRUVED plus low fat) groups throughout intervention...........................................................106

Abundance profile of (A) Ceramide, (B) GlcCer, and (C) LacCer in all subjects.........................108

Heat map representation of ceramide and glycosylated ceramide changes with intervention......109

Changes in circulating ceramides during intervention...............................................................110

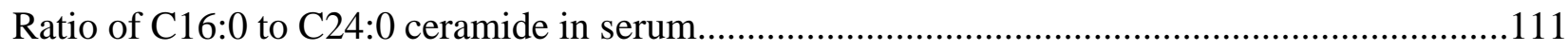

Changes in circulating monohexosylceramides (GlcCer) during intervention..............................112

Changes in circulating lactosylceramides (LacCer) during intervention.......................................114

Random regression analysis between serum concentrations of (A) C24:0 ceramide and monohexosylceramide (GlcCer), and (B) C24:0 GlcCer and lactosylceramide (LacCer)...........115

Correlation between Original PSQI and "shortPSQI".......................................133

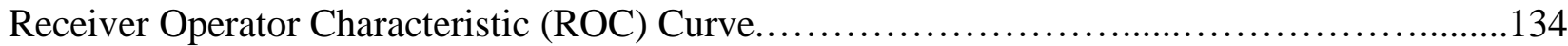


Chapter I: Background 


\section{Impact of Nutrition and Disease}

Despite recent advances in prevention and treatment, obesity and cardiovascular disease (CVD) still account for high morbidity and mortality in the United States (U.S.) [1, 2]. Although there may be a contribution of genetic factors related to disease [3], environmental factors such as weight gain, physical inactivity, and poor dietary behaviors play a large role in causality and management [4]. Dietary modification has long been a cornerstone of disease prevention and more specifically, dietary priorities have focused on increasing fruit and vegetable consumption and limiting total fat and sugar intake [5]. Current nutritional guidelines recommend a diet that includes at least five servings of fruits and vegetables per day $[6,7]$. These recommendations are based on scientific findings that affirm that fruits and vegetables contain components such as plant proteins, fiber, potassium, magnesium, folate, and compounds with antioxidant capacity. The consumption of these nutrients may prevent chronic disease and reduce the risk of metabolic syndrome (MetS) components [8-10]. Ford et al. used data from the Third National Health and Nutrition Examination Survey (1988-1994) to compare antioxidant levels and fruit and vegetable consumption in U.S. adults aged 20 years or older with and without MetS [11]. After adjustment of age, sex, race or ethnicity, education, smoking status and other covariates, researchers found that those with the MetS had significantly lower antioxidant levels than those without the MetS. Researchers also found that those with MetS had significantly lower consumption of fruit and vegetables. Similar findings were observed in a cross-sectional study of 486 Tehrani female teachers (40-60y), where fruit and vegetable intake was analyzed in relationship to C-reactive protein (CRP) concentrations, a marker of inflammation in the body, and the prevalence of MetS [12]. There were inverse associations with fruit and vegetable intake and CRP concentrations, even after controlling for age, body mass index (BMI) and waist circumference. Additionally, 
participants in the highest quintiles of fruit and vegetable intake (mean $362 \mathrm{~g} / \mathrm{d}$ and $231 \mathrm{~g} / \mathrm{d}$ respectively) had a 34\% and 30\% lower chance of having MetS compared to those in the lowest quintiles (mean $99 \mathrm{~g}$ fruit/d and $121 \mathrm{~g}$ vegetables/d). Lastly, a randomized controlled trial investigating nonsmoking men consuming 2 or more servings of fruit and vegetables a day for 4 weeks, showed that subjects who consumed 8 or more servings daily had significantly reduced CRP values at week 8 compared to subjects only consuming 2 servings a day [13]. These findings support investigation of the understanding of overall dietary quality, with increased fruit and vegetable consumption, on disease markers.

In addition to fruit and vegetable consumption, studies have investigated the effect of specific diets on metabolic parameters. An increasingly popular diet that is currently used to decrease cardiovascular events and mortality in adults is the Mediterranean diet [14]. This diet is characterized by increased consumption of olive oil, fish, whole grains, fruits and vegetables, olives, and nuts [15]. Recently, studies have examined the efficacy of the Mediterranean diet to decrease cardiovascular risk factors [16-18]. From 2001 to 2004, 180 participants with MetS at a university hospital in Italy were randomized into either a Mediterranean-style diet or a standard diet [17]. Measurements included endothelial function as a measure of blood pressure, insulin sensitivity, CRP levels and other inflammatory markers (Interleukin (IL)-6, IL-7, and IL-18). After 2 years, patients consuming the Mediterranean-style diet had significantly reduced inflammatory markers, decreased insulin sensitivity, improved endothelial function, and showed less prevalence of MetS compared to the control group. Similarly, while studying obese children (3-10y) and adolescents (10-18y) in Mexico, researchers demonstrated that participants assigned to a Mediterranean-style diet for 16-weeks had significantly lowered BMI, fat mass, glucose, triglycerides, total cholesterol, and LDL compared to a standard diet [16]. The Dietary 
Approaches to Stop Hypertension (DASH), may also be considered an effective eating plan to reduce metabolic risks because it is rich in fruits and vegetables, limits sodium intake, and has low amount of dairy fat [19]. In a randomized, controlled, outpatient trial of 116 patients with MetS, researchers found that individuals on the DASH diet had increased high density lipoprotein (HDL) cholesterol, and lowered triglycerides, systolic and diastolic blood pressure, weight, and fasting blood glucose compared to participants put on a control diet [20]. Altogether, these two diets, as well as the Dietary Guidelines for Americans [7], support a diet high in fruit and vegetables, low in saturated fat, and low in refined carbohydrates to improve metabolic parameters that increase risk of disease. However, the question arises whether the variable components of a healthy diet (i.e. high fruits and vegetables, low-fat, or low-refined carbohydrate) exert different beneficial effects on risk factors for disease. Some researchers have shown that a diet low in glycemic load, containing less carbohydrates from high-glycemic index foods, represents an alternative to low-fat diets [21] that have been recommended for weight-loss and improvement of other metabolic functions [22]. Thus more research is needed to investigate this underlying question.

\section{Dietary Behaviors among College Students and Appropriate Interventions}

Although proper nutrition is integral to health, the majority of the U.S. population are not meeting dietary recommendations [23]. In a dietary surveillance study, nearly the entire U.S. population consumed a diet with fewer vegetables and whole grains than recommended. The majority also under consumed fruits, milk, and oils compared to recommendations [23]. After investigating differences among age groups, young adults (19-30 years old) were most likely to have insufficient intakes. In 2003, the Behavioral Risk Factor Surveillance System (BRFSS) reported that $43 \%$ of young adults (18-24) had little to no physical activity and $78 \%$ reported 
consuming less than 5 servings of fruits and vegetables per day [24]. Moreover, young adults are an important target audience for public health nutrition because the transition from adolescence to adulthood can increase the risk of developing obesity [25]. During this time, many young adults experience landmark life events such as new jobs, more financial independence, childbearing, and entering post-secondary education. The university years are considered a critical time for shaping behaviors and providing dietary intervention because young adults are establishing independent lifestyle and adopting self-determined eating behaviors [26]. The food patterns established during college are likely to be maintained for life and can influence the health of the student and their future families [27]. However, this time period can pose significant changes to healthy eating because some students have difficulty purchasing and preparing meals independently, managing their eating schedules, or may have limited access to healthy foods and limited support for eating well [28-30]. Research has shown that the average college student consumes 1 serving of fruit, 1.5 serving of vegetables, and 1.4 servings of whole grains daily [29, 31]. Given the evidence of poor behavioral choices among young adults, additional intervention studies are needed to evaluate the efficacy of promoting healthy eating in order to decrease the risk of immediate and consequent nutrition-related morbidities.

Traditionally, dietary behavior change has been done through expert-led, face-to-face education via individual or group sessions [32] and literature indicates that adults sustain positive eating behaviors after undergoing face-to-face education $[33,34]$. Aspects of this interpersonal technique that aid in the effectiveness of dietary change are self-monitoring of intake, tailored feedback, goal setting based on diet assessment, and reinforcement of behavior [35]. Additionally, personalizing nutrition educational messages compared to generic group nutrition counseling have been shown to be more effective [34]. Tailored messages are individualized 
based on assessment of personal behaviors, psycho-social characteristics, and are specific to targeted outcomes $[36,37]$. In essence, people respond to messages that are relevant to themselves [35]. Wright et al. investigated the use of tailored, iterative, printed dietary feedback over the course of 3-months in middle-aged adults with cardiovascular risk factors to improve dietary behavior [38]. Comparing the tailored feedback to small group nutrition education session, they found dietary feedback was more effective in improving fruit and saturated fat intake. Other studies have reported positive effects of tailored interventions on fruit and vegetable intake [39]. Currently, a randomized controlled trial is being implemented to evaluate the effectiveness of using tailored feedback and text messages to motivate changes in fruit, vegetable, and junk food consumption among adults [35]. Thus studies suggest that tailored interventions are a promising strategy for health education and dietary behavior change, however the duration of such an intervention program to be effective and sustained continues to be investigated.

In addition to tailored interventions, theory-driven approaches that incorporate individual beliefs, attitudes, and perceived intake levels are helpful in reaching young adults with low tendency for dietary behavior change [40]. In particular, most nutrition intervention in college students have been informed by the Social Cognitive Theory (SCT) [41-43]. This theory postulates that change is dependent on the interchange between the individual's environment (i.e. access, availability), personal (i.e. self-efficacy, knowledge), and behavioral (i.e. skills) factors [44]. Additionally, the SCT is considered most appropriate for university students because of its focus on self-efficacy and enhancing behavioral skills [40, 45]. Results from studies targeting students' nutritional dietary habits yielded improvements in eating habits post intervention [41, 43, 46]. However, more research is needed that examines detailed assessment and tailoring of 
theoretical frameworks to improve nutrition among university students.

\section{Gut Microbiome and Nutrition}

As indicated in the previous studies, diet has an important role in the pathology and treatment of disease. However with new advances in technology over the past decade, the microbial communities on all surfaces of the human body have gained considerable attention and represent a new frontier in medical research. In adults, the combined microbial population exceeds 100 trillion cells (approximately $2 \mathrm{~kg}$ in cell mass), which is about 10 times the total of cells of the human body [47]. The vast majority of these microbes inhabit our gastrointestinal tract, with the greatest amount residing in the distal gut. These microbiota serves as an interface between food, the different fuels absorbed, and the human body. They help process components of otherwise indigestible dietary contributions to our diet and synthesize vitamins and essential amino acids [48]. The microbial population in our distal gut is estimated to contain over 100 times as many genes as our own, therefore our body can be considered superorganismal, where metabolic interactions occur between our own genetic landscape and the genes affiliated with the microbiota (microbiome). The progress made with 16S ribosomal RNA (rRNA) sequences have disclosed significant differences in microbial populations between healthy adults [49], and these differences appears to play a role in human disease. Over the past decade, researchers have linked an imbalanced gut (dysbiosis) with obesity, diabetes, atherosclerosis, and MetS risk [50].

The underlying mechanism of obesity and inflammation from the gut microbiota is conflicting, yet many mechanisms have been identified. For example, microbiota are connected to suppression or elevation of fasting-induced adipose factor (Fiaf), which is a circulating inhibitor of lipoprotein lipase [51]. When germ-free mice were fed a Western diet, they were resistant to diet-induced obesity and demonstrated higher levels of intestinal Fiaf. On the other 
hand, when germ-free mice were colonized with the microbiota of wild-type mice, they displayed suppressed levels of Fiaf and had greater fat deposition [52]. Additionally, the microbiota colonize the non-adherent mucus layer of the GI tract, and may also be responsible for reduced barrier integrity and systemic endotoxemia during obesity. In particular, gut microbiota has been linked to low-grade inflammation through activation of innate immunity via lipopolysaccharide (LPS) - toll like receptor (TLR) - 4 axis [53]. After 16 weeks of being fed a high-fat diet (45\% kcal from fat), wild-type mice showed a high association between ileal TNFalpha gene expression with weight gain and an increase in fasting glucose [54]. In comparison the germ-free mice fed the same diet showed attenuation of adiposity, intestinal inflammation and metabolic disturbances. Together, these data provide evidence that alterations in gut microbiota by diet-induced obesity can cause barrier disruptions to the GI tract and inflammation.

With high rates of obesity and its associated metabolic diseases, research has investigated the consumption of a Western diet, rich in fat and simple sugars, on microbial dysbiosis [55] [56]. High intake of fat and protein is associated with Bacteroides, whereas increases in Prevotella are associated with high fiber intake [57]. Evidence provided by Serino et al. showed that after genetically identical mice consumed a high-fat, carbohydrate-free diet, a distinct group developed insulin resistance, gut permeability, increased endotoxemia, and systemic/adipose tissue inflammation [58]. These metabolic adaptations were associated with microbial changes of an inverted Bacteroidetes to Firmicutes ratio, and a reduction in the Lachnospiraceae family. Decreased total carbohydrate in weight loss human trials accompany reduction in fiber and indigestible starch that provide fermentation for healthy commensal bacteria. The provision of these diets to obese individuals resulted in decreased concentrations of short chain fatty acids 
(SCFAs), together with decreases in butyrate-producing Lachnospiraceae [59]. These data provide evidence that alterations in gut microbiota is to a large extent modulated by diet, which can may cause barrier disruptions to the gastrointestinal tract and inflammation.

Although perturbations of the microbiota may lead to metabolic dysfunction, there is evidence linking certain dietary components to beneficial bacteria that lead to energy balance and decreased inflammation [60]. The mechanism occurs when microbiota metabolize indigestible polysaccharides that escape digestion in the upper part of the gastrointestinal tract because of enzymes encoded in the bacterial genome [61]. An end result of this metabolism are SCFAs (i.e. acetic, propionic and butyric acid) that act as carbon sources for the host. Additionally, SCFAs inhibit autophagy in gut epithelial enterocytes [62], reduce proinflammatory cytokine production [63-65], and stimulate gut hormones involved in reduced food intake [66]. Based on these microbial metabolites, dietary interventions present huge potential in therapeutic strategy for gut dysbiosis.

Common types of dietary manipulation on the gut dysbiosis have occurred through consumption of either probiotics (live microorganisms that enrich probiotic species in the intestines) or prebiotics (dietary components that support growth and or activity of one or more microbial species) [67]. After administration of a prebiotic (oligofructose) to genetically obese mice, decreases in fat mass, LPS, and improved lipid and glucose metabolism via a mechanism that was associated with reduction in hepatic glucose-6-phosphatase expression was found [68]. Additionally, researchers observed improved gut barrier function. The impact of oligofructose on gut microbiota showed increases in abundance of Akkermansia muciniphila. Furthermore, the same research group saw decreases in A. muciniphila in obese and type 2 DM mice [68]. When researchers gave A. muciniphila to an obese animal, they observed improved glucose tolerance 
[69]. Although these studies show that dietary supplementation with probiotics and prebiotics have beneficial effects on obesity $[70,71]$, variations in outcomes have been reported, which are likely due to the use of different bacteria strains, the route of administration and the duration of the studies [72].

When weight loss in humans occurred by either a carbohydrate or fat-restricted diet, changes in the microbiome resembled that of lean individuals with increases in Bacteroidetes [73]. Although data shows that composition of microbiota in a healthy subjects differs from that of an unhealthy subject, it is still not clear what represents a healthy microbial profile or what dietary interventions are needed to manipulate gut microbiota for obesity and MetS management. One such study investigated the effect of five experimental diets in subjects (ages 43-68, n=88) who were "at-risk" of MetS and consumed a particular diet for 4 weeks [74]. The five diets consisted of the following: high monounsaturated fat/high glycemic index; high monounsaturated fat/low glycemic index; high carbohydrate/high glycemic index; high carbohydrate/low glycemic index; and high saturated fat. Results after 24 weeks showed that the groups who increased carbohydrate and reduced dietary fat increased numbers of Bacteroides, which were inversely associated with body weight $(r=-0.64)$ [74]. This study and others [75] provide evidence that high carbohydrate diets may benefit the alterations of human fecal bacteria to induce weight-loss in individuals at risk of disease. However, the mechanisms between diet and the microbiome needs to be further elucidated, therefore more controlled human studies regarding diet change are necessary.

\section{Metagenomics, Translational Research, and Team Science}

Although our microbial ecosystem contributes to health and disease, there is limited ability to infer organismal function from gene sequences by the preferential polymerase chain 
reaction (PCR) amplification of the 16S rRNA unit. Simply put, this technique determines "who is there" when examining our gut microbiota. However, researchers are shifting their focus towards understanding "what are they doing", which is termed metagenomics. In knowing the core functional profile of the gut microbiota, researchers can begin to explore how different microbes produce changes in metabolites throughout the host. In order to identify these biologically variations, studies are combing metabolomic analyses (the characterization by mass spectrometry, NMR, or other analytical methods of metabolites generated by one or more organisms in a given physiological and environmental context) with the assessment of metabolic features of microbial communities [76]. So far in the metabolomics field, researchers have identified serum amino acids, mostly branched-chain amino acids (BCAAs) [77] and triacylglycerols with low carbons and double bonds [78] to be associated with insulin resistance and future metabolic abnormalities. Additionally, there is evidence that links the gut microbiome and human metabolic health [79]. For example, researchers identified bacterial species that drove the association between biosynthesis of BCAAs and insulin resistance [80]. These findings provide exciting new prospects for personalized medicine, particularly in studies that determine mechanisms by which microbes affect production of metabolites from diet.

Undoubtedly, the interplay between gut microbiome and host and its modulation by nutrition presents many challenges to overcome. However, it provides opportunity for advancement of scientific knowledge from "bench to bedside" [81] and bidges basic science discovery to public health impact, or what is commonly referred to as "translational research" [82]. Furthermore, this type of large-scale research will promote cross-disciplinary collaboration, which is a useful but also essential strategy for ameliorating disease outcomes [83] in hopes of sustaining the future among a young generation. 


\section{Conclusion}

In conclusion, eating patterns and dietary behaviors play a huge role in the development and treatment of disease. Proper nutrition among individuals in the U.S. is poor, especially the consumption of fruit and vegetables, which falls far below half of what one should be consuming daily. Considering the multitude of personal, social, and environmental factors that college students face, they may demonstrate unhealthier lifestyles and poorer eating habits, increasing their risk of disease. For that reason, more tailored, theory-driven interventions are warranted to reduce future cardiovascular events and CVD in this population.

In addition to dietary manipulation for prevention of disease, expansive work on the microbiome over the past decade has shown how diet influences microbial composition that can either increase or improve metabolic dysfunction, yet few studies have targeted the college student population. Lastly, there is agreement that more research on the marriage of metabolomics and microbiome needs to be done to elucidate the mechanisms between the genes contributed by our body and those of the microbes living in our gut. Therefore new strategies, technologies, and multi-disciplinary approaches must be employed to evaluate the efficacy of a nutritional intervention on many metabolic parameters in the college student population.

\section{Study Background, Objectives, and Hypotheses}

It is tempting to speculate that reversing a Western Diet (high in protein, fat, and sugar) to one with enrichment of indigestible plant polysaccharides and other healthy diet components would shift microbial composition and decrease metabolic dysfunction and disease progression in young adults attending college. This question is explored by the FRUVEDomics pilot project (Clinical Trial Record 1409433435) that stems from a larger United States Department of Agriculture (USDA) project called 'Get FRUVED'. 'Get FRUVED' is a funded Agriculture and 
Food Research Initiative (AFRI) that uses community-based participatory research, with social marketing, and environmental change to help older adolescents effectively managing their weight during their first year of college. This obesity prevention campaign is a collaboration between 8 universities, including West Virginia University (WVU), which focuses on promoting healthy lifestyle behaviors (increase fruit and vegetable intake, manage stress, and encourage physical activity) among first year students at-risk for weight gain. College juniors and seniors were recruited and trained to create and host healthy events on campus and assess the campus environment, with the intention of impacting its physical environment for well-being in the longterm. While the overall umbrella of the 'Get FRUVED' project was designed to influence the environmental factor of the SCT in promoting dietary change among college students, the FRUVEDomics pilot study was created to focus on a personal level nutrition intervention and its impact on various metabolic parameters.

In the FRUVEDomics project, the first objective was to investigate the nutritional behaviors of young adults attending a university, after implementation of a dietary intervention centered on the USDA Dietary Guidelines for Americans 2010 (Chapter 2). The second objective was to examine changes in the microbiome after the eight week dietary manipulation and to explore relationships among dietary components and serum amino acids (Chapter 3). Hypotheses of the primary objectives were that adoption of the national recommendations would 1) improve nutritional behaviors that would result in enhanced metabolic health and 2) shift microbial populations associated with low grade inflammation and reduced adiposity. Lastly, a secondary objective was to use lipidomics analysis to investigate the nutritional impact on circulating ceramides and glycosylated ceramides (Chapter 4) where we speculated that a healthy diet would lower plasma concentrations of inflammatory mediators and ceramides, thus improve health. 
Chapter II: Efficacy of a Personalized Nutritional Intervention on Food Consumption and Metabolic Parameters in Young Adults 


\section{Abstract \\ Efficacy of a Personalized Nutritional Intervention on Food Consumption and Metabolic Parameters in Young Adults: FRUVEDomics Pilot Study}

OA Famodu ${ }^{1}$, ML Barr ${ }^{1}$, I Holásková ${ }^{1}$, PJ Murray ${ }^{2}$, CF Cuff $^{3}$, JW McFadden ${ }^{1}$, MT Downes ${ }^{4}$, SE Colby $^{5}$, IM Olfert $^{6}$, MD Olfert ${ }^{1}$

West Virginia University, Davis College of Agriculture, Natural Resources and Design, Division of Animal and Nutritional Sciences in Department of Human Nutrition \& Food; West Virginia

University, School of Medicine Morgantown, WV; University of Tennessee, Knoxville

Background: Rates of disease are increasing in young adults (years 18-28), further creating a need for interventions that will improve later quality of life. Modifying diet can help assist in health maintenance and disease prevention. Considerable research on dietary behavior change for the promotion of health has been done, however, successful theory-based, dietary behavioral interventions for young adults who follow poor lifestyle habits are limited. The objective is to evaluate the impact of the FRUVEDomics pilot study on food consumption and metabolic parameters on young adults attending a university.

Methods: An 8-week free-living dietary intervention, based on the 2010 USDA Dietary Guidelines for Americans and driven by the Social Cognitive Theory, was conducted in young adults (18-28 years old) at West Virginia University. Subjects $(n=36)$ were randomized into one of three nutritional intervention groups; 1) "FRUVED" (50\% fruit \& vegetable), 2)

"FRUVED+LRC" (50\% fruit \& vegetable plus low refined carbohydrate), and 3) "FRUVED+LF" (50\% fruit \& vegetable plus low fat). Anthropometrics, surveys, venous blood and stool samples, and body composition were collected before and after the intervention. Group nutrition education including basic nutrition for the prescribed intervention, culinary tool kit distribution, and grocery shopping tips was successfully delivered to each group prior to the start of the intervention. Participants underwent individual weekly consultations with a Registered Dietitian Nutritionist using food logs, food pictures, and receipt management to assess adherence.

Results: No changes were seen in total caloric consumption, physical activity, stress or sleep. Individuals of all groups significantly decreased empty caloric foods (low nutrient content and calorie dense) $(\mathrm{p}<0.0001)$ and increased total calories from carbohydrates $(\mathrm{p}=0.0001)$, fiber $(p<0.001)$, and cups of fruit and vegetable $(p<0.0001)$ consumption after 8 weeks, with no differences among groups in these dietary components. Improvements in waist circumference and systolic blood pressure were seen in all groups of those who complied with the intervention $(\mathrm{p}<0.01$ and $\mathrm{p}<0.05$ respectively).

Conclusions: The dietary intervention established around the population-based recommendations for Americans resulted in meaningful changes to dietary behaviors that improved metabolic parameters in young adults attending a university. 


\section{Introduction}

Western-style living creates an "obesogenic" environment due to constant exposure to easily accessible, inexpensive, and high portioning of energy-dense foods and sedentary life, resulting in an epidemic of metabolic disorders. In the United States (U.S.), overweight and obesity in young adults (ages 20-39) was estimated to be $67.1 \%$ in men and $55.8 \%$ in women [2]. The prevalence of metabolic syndrome (MetS), a group of biochemical and anthropometric indices that increases risk of cardiovascular disease (CVD) is small among the young adult population [84]. However, a recent study reported that $77 \%$ of the male and $54 \%$ of the female college students had at least one MetS criterion [85], indicating the need of early identification and screening of metabolic dysfunction among a seemingly low-risk population. Furthermore, the continued rise in obesity and risk factors for CVD exacerbates medical problems, contributes to increased healthcare costs, and decreases quality of life, further creating a need for interventions to focus on younger populations.

Attending a university is a transformational time for young adults; they are making more independent lifestyle choices (drug/alcohol use, sleep patterns, exercise, and diet habits) [26]. However, college life can be coupled with poor time management, increased stress, and poor food availability and choices, leading to unhealthy behaviors and unwanted weight gain [86, 87]. It has been reported that $43 \%$ of young adults (18-24) had little to no physical activity and $78 \%$ reported consuming less than the recommended 5 servings of fruits and vegetables per day [24]. Given the high rates overweight/obesity and poor lifestyle behaviors, this young adult population may be at greater risk of CVD and other medical problems than other young generations [88]. Thus, primary preventions targeting health behaviors in the young adult, college population may help reduce rates of disease and prevent some of the long-term health consequences [89]. 
Promotion of healthy eating behavior is among the many priorities of governing bodies to prevent disease [5]. The 2010 Dietary Guidelines for Americans, created by the U.S. Department of Agriculture (USDA) and the U.S. Health and Human Resources (HHS), are expert opinion food consumption recommendations for optimal health for individuals residing in the U.S. [7]. The recommendations are centered around major "action steps" [90]: 1) maintain energy balance through reducing overall energy intake and increasing physical activity; 2) focus on nutrient dense foods such as vegetables, fruits, whole grains, nuts and seeds, and low-fat milk and milk products; and 3) reduce added sugars, solid fats, and sodium. In the U.S., the majority of the population consumes inadequate fruit, vegetables and whole grains and is coupled with overconsumption of solid fats, added sugars, and alcoholic beverages [23]. Furthermore, young adults consume insufficient intake of fruits and vegetables [23].

Researchers suggest that conventional diets such as low refined carbohydrate [91] and low fat [92] diets improve metabolic parameters and several studies have compared their health benefits $[93,94]$. However, given that a diet only focusing on increased fruit and vegetable consumption has been beneficial for health in various populations [95], few researchers have compared it alone to the addition of the a low-fat or low-carbohydrate diet recommendations to investigate if additional health outcome effects occur [96], especially in young adults. Moreover, little is known about the best approaches to improve dietary consumption using the populationbased guidelines in young adults attending a university.

For most young adults, adopting a diet consistent with recommended guidelines will require major behavioral changes. These changes are successful with personal level interventions instead of public health programs [97] because they assist in raising awareness of key health messages, increasing motivation to perform the behavior, and are relevant to the individual [98]. 
Additionally, tailored interventions are able to utilize several factors known to be important for dietary change, which include self-assessment of intake, tailored feedback, goal setting based on assessment, and reinforcement of behaviors [32]. Moreover, notable results have occurred in studies using personal level interventions in less than 6 months [99]. However, researchers have shown that adopting a behavior can range from 18-254 days [100] and requires incremental strengthening between a cue or situation and an action (i.e. repetition within a specific context) [101]. Most researchers also agree that habits originate when goals are being pursued that yield desired outcomes [102]. However, in order to develop tailored strategies that use habit-forming cues to help individuals adopt and maintain healthful dietary patterns, integration of theoretical models are needed to identify relevant determinants of successful behavior change [103].

Using theoretical foundations is key for designing and implementing nutrition counseling protocols and enhancing counseling effectiveness $[28,104]$. The Social Cognitive Theory (SCT) suggests that cognitive learning takes places by learning from one's social context and that there are reciprocal relationships between the cognitive factors (i.e. knowledge, attitudes, motivation, and self-efficacy), behavioral factors (i.e. skills), and environmental factors (i.e. cost, availability and social support) [44]. Practical implications for the SCT in college students undergoing a dietary intervention are to enhance students' self-efficacy and behavioral skills to eat healthy [105] and few studies have documented the efficacy in using it for nutrition interventions among young adults attending college [43, 99]. Nutrition counseling studies using these theories to change dietary measures are needed to validate the efficacy in the young adult population.

To our knowledge, intense and tailored dietary interventions driven by theory and created to attenuate morbidity and mortality risk are limited in young adult population attending college. West Virginia University (WVU) students may be considered a higher-risk population because 
they are located in the heart of the Appalachian Region, where incidences of CVD, obesity and type 2 diabetes (T2DM) are above the national average [106-109]. Furthermore, estimated proportion of adults ( $\geq 18$ years) consuming at least five servings of fruit and vegetables per day in WV is only $16.2 \%$ [110]. Given that food patterns established during college may be maintained for life and have long-lasting influences on college students' future health and their future families [111], the FRUVEDomics pilot study (Clinical Trial Record 1409433435) was designed to improve nutritional habits among WVU college-age students using the USDA 2010 Dietary Guidelines for Americans and face-to-face contact with a nutrition professional. The purpose of this research study is to evaluate the impact of the FRUVEDomics pilot study on food consumption and metabolic parameters on young adults attending a university.

\section{Methods}

\section{Subjects, Setting, and Recruitment}

The FRUVEDomics pilot study was a randomized, pre-post trial conducted in 36 young adults (15 men and 21 women; 18-30 years of age) from WVU in the winter of 2015. Subjects were recruited as a subproject from a larger USDA project called 'Get FRUVED'. Get FRUVED is a USDA funded, Agriculture and Food Research Initiative that uses community-based participatory research, with social marketing, and environmental change to help older adolescents effectively manage their weight during their first year of college. This obesity prevention campaign is a collaboration between 8 universities, including WVU, which focuses on promoting healthy lifestyle behaviors (increase fruit and vegetable intake, manage stress, and encourage physical activity) among first year students at risk for weight gain. To promote healthier lifestyles, juniors and seniors were recruited and trained to create and host healthy events on campus and assess the campus environment, with the intention of impacting its 
physical environment in the long-term for well-being. While the overall umbrella of 'Get FRUVED' project was designed to change the environmental component of the SCT, the FRUVEDomics pilot study was created to focus on a personal level nutrition intervention and its impact on metabolic parameters.

Recruitment was done by word-of-mouth, campus-wide flyers, and announcements posted to WVU's electronic news page starting in November 2014. Inclusion criteria included: age 18-30, free from chronic disease, have no diagnosis or treatment of a serious mental or behavioral disorder within the past year, not pregnant, and have a BMI greater than 18.5. Exclusion criteria included current participation in another dietary study or following a specific diet regimen. Individuals who met the criteria were asked to conduct a phone interview with a trained researcher to discuss the study protocol and exclusion criteria. Participation in this freeliving design required compliance, thus the researchers were looking for individuals in the "preparation" stage of the Trans Theoretical Model, who would commit to an eight week healthy diet intervention. An assessment of this was ascertained by the following question during the phone call: "Are you willing and able to adhere to a nutritional intervention consisting of half of your plate of fruits and vegetables for 8 weeks with an addition of either a low-fat or a low processed carbohydrate component?" If the participant answered yes and met the inclusion criteria, they were asked to come in for assessments. Approval was obtained from WVU Institutional Review Board and informed consent was collected from each subject prior to enrollment.

\section{Outcome Measures}

Clinical and nutritional history as well as physical parameters were obtained on all participants. To reduce error, all measurements were taken by a trained graduate student and the 
same student conducted the measurements before and after the intervention. Weight was measured using digital scales (SECA 874) and recorded to the nearest $0.1 \mathrm{~kg}$ while the subjects were minimally clothed and without shoes. Height to the nearest $0.1 \mathrm{~cm}$ was measured in a standing position without shoes using a Stadiometer (SECA 213). Subjects were told to relax their shoulders and face forward, looking straight with their back against the wall. Body mass index (BMI) was calculated using the measured height and weight. Blood pressure was measured twice, with a two minute interval between each measurement, using an Omron digital blood pressure cuff after the subject sat up straight for five minutes with relaxed, un-crossed legs. Measurements of body composition were taken with a BodPod (CosMed USA Inc., Chicago, IL) while the individual was dressed in minimal, tight fitting clothing. Waist circumference was measured at the narrowest level and hip circumference was measured at the maximum level over light clothing using a Gulick tape meter. Neck circumference was taken immediately below the laryngeal prominence (Adam's Apple) with the participant's body held erect and eyes facing forward [112]. All measurements were recorded to the $0.1 \mathrm{~cm}$ after being taken twice and averaged, with the averages used for analysis.

Ten-hour fasting blood samples were collected by venipuncture and centrifuged 3,400 $\times$ $g$ for 10 min at $4^{\circ} \mathrm{C}$ after coagulating at room temperature for 30 minutes. Following centrifugation, serum was decanted and stored at $-80^{\circ} \mathrm{C}$ until analysis. Assays for a complete metabolic panel (triglycerides, high density lipoprotein (HDL), calculated low density lipoprotein (LDL), total cholesterol, insulin, fasting blood glucose), hemoglobin A1c and highsensitivity C-reactive protein (hs-CRP), were performed by the WVU's Ruby Memorial Hospital Clinical Laboratory. Ratios of total cholesterol to HDL cholesterol and LDL cholesterol to HDL cholesterol were calculated. The homeostatic model assessment to quantify insulin resistance 
(HOMA-IR) was calculated as the product of fasting plasma insulin in microunits and fasting blood glucose level in millimoles per liter, divided by 22.5 [113].

Physical activity, sleep hours, and perceived stress were determined through subjective and objective measures before and after the 8 week intervention. Activity was measured by having the participant wear an accelerometer (ActiGraph GT3X, Actilife 6.0 Software, Pensacola, FL) around their waist for one week before the start and at the end of the intervention with specific instructions provided. Wear time compliance criteria required participants to wear the device for three valid days. Non-wear time was defined as $\geq 60$ repeated minutes of zero activity counts. Average steps per day were calculated using age specific cut-points by Freedson and colleagues cut-points [114]. Hours of sleep at night were determined by asking the subject, “during the past month, how many hours of actual sleep did you get a night?", obtained from the Pittsburgh Sleep Quality Index (PSQI) [115]. The 10-item Cohen's Perceived Stress Score (PSS) [116] was also administered.

\section{Randomization and Intervention Groups}

After all subjects were recruited and baseline measurements obtained, they were randomized evenly into one of 3 groups (n=12/group): 1) “FRUVED” diet (50\% fruit \& vegetable intake), 2) "FRUVED+LRC" (50\% fruit \& vegetable plus with low-refined carbohydrate intake), and 3) "FRUVED+LF" (50\% fruit \& vegetable plus low-fat intake). A description of each of the dietary interventions can be seen in Table 1.

Male participants were first randomized to ensure distribution of sex into the three dietary arms. Next, if a female or male partner was enrolled in the study (i.e. roommates, relatives, romantic partners) then they were put in the same group. After all male participants, including 
their partner, were placed into groups, then remaining female participants were randomized and the same procedure was used if they had a female partner in the study.

\section{Nutrition Education}

Prior to the start of the 8 week intervention and right before spring break (January to March 2015), the subjects were required to attend a 2-hour nutrition educational session, led by a Registered Dietitian Nutritionist (RDN), with the other participants in their group. Participants were trained on how to build a healthy menu based on their prescribed diet and taught how to read food labels. Education also included portion estimations, budgeting and grocery shopping tips, ways to include more fruits and vegetables into the diet, and how to use the food log and take pictures of their food. In addition to the education, subjects were given diet plans in a binder that included a list of allowable food items, menus and recipes, along with other helpful information to guide them throughout the intervention. A culinary toolkit consisting of knives, a cutting board, plastic ware, plastic bags, and other kitchen utensils were received by each participant to help apply what they learned in the education session to cooking and preparing meals at home. Lastly, all participants received instructions to maintain pre-intervention levels of physical activity, sleep behaviors, and were giving resources to manage stress throughout the entire duration of the study.

\section{Dietary Evaluation and Personalized Counseling}

In accordance with the 2010 USDA Dietary Guidelines for Americans, each participant was given a caloric intake to maintain for the 8-weeks that was based off their current weight, age, and physical activity status. The portions for each food group, according to USDA's MyPlate, were calculated for each participant from the computer software Nutritionist Pro ${ }^{\mathrm{TM}}$ (Axxya Systems LLC, Redmond, WA). Participants were evaluated and educated every week by 
a one-on-one counseling session with a RDN. This intense intervention helped shape individual knowledge, motivation, and self-efficacy. The same RDN met with all participants for their one hour counseling sessions weekly to maintain consistency. Each session consisted of four sections; 1) evaluate compliance with intervention assignment and review prior week goals 2) conduct a 24-hour dietary recall; 3) provide personalized education; and 4) set goals.

At the beginning of the counseling sessions, the participant was asked to rate "how well they are complying with prescribed diet" on a continuum between 0 (no compliance) and 100 (full compliance). Next, the RDN evaluated the participant's food pictures, receipts, food log and other health concerns or changes. Then the RDN conducted a dietary recall, asking the participant to remember what he/she ate and drank the previous 24 hours.

Following compliance measurements, dietary advice was tailored to each participant based on the weekly nutrition logs completed by the participant, the food photographs, and the previous 24 hour diet recall assessment using Nutritionist Pro ${ }^{\mathrm{TM}}$ (Axxya Systems LLC, Redmond, WA). The software determined total calorie content, empty calorie content (defined as calories that come from solid fats and added sugars), portions, and macro- and micronutrients that were consumed compared to the isocaloric recommended plan (Figure 1). If the participant fell below or above the recommendations, the RDN would use food replicas (Nasco Fort Atkinson, WI) during the counseling session to show portion size estimations that matched personal diet prescription.

At the end of each session, the participant made one or two specific, measureable, and attainable goals for the next week and were encouraged to sign-up for a grocery or dining hall tour, or sit in on a food demonstration to help address any environmental barriers. To compensate the participants for their time and effort in the study, incentives in the amount of $\$ 25$ 
was given at the baseline assessment, during 6 counseling sessions with the RDN, along with a $\$ 50$ incentive given at the post assessment. Total reimbursement was $\$ 225$ for those who completed all assessments and 8 weekly counseling sessions.

As soon as the session ended and after full assessment of all dietary factors, the RDN rated the participant on the same compliance continuum scale given to the participant at the beginning of the meeting. This scoring system helped the researchers evaluate whether the participant would be excluded from the metabolic parameter analyses for not fulfilling the dietary recommendations.

\section{Statistical methods}

Data are presented as means with standard deviations (SD) unless stated otherwise. Summary statistics were calculated for demographic characteristics and an analysis of variance (ANOVA) was used to examine difference among the groups at baseline. All continuous outcome distributions were examined and transformed if necessary, which included logtransformation or square-root. A repeated measures ANOVA was used to assess all dietary, anthropometric, and biochemical differences among groups, over time, and the group by time interaction. Post hoc multiple comparisons with Tukey adjustment was utilized. $\mathrm{P}<0.05$ was considered statistically significant and all analyses were conducted using SAS (SAS®, Version 9.3, SAS Institute Inc., Cary, NC, Copyright @2002-2010) and JMP (JMP®, Version Pro 11, SAS Institute Inc. Cary, NC, Copyright (C2013). 


\section{Results}

\section{Descriptive Results}

Figure 2 illustrates the flow of the participants through the intervention. The average age of the thirty-six participants who completed the 8-week intervention was 23.2 years (Table 2). The study sample was primarily white (64\%), from the Appalachian region (53\%), and female (58\%) (Figure 1). Minority and male enrollment was $36 \%$ and $42 \%$ respectively, with an equal spread of African-Americans, Hispanics and Asians. Average BMI was 27.2 (SD 5.96) and 50\% of the participants were either overweight or obese. Forty-five percent of the participants were undergraduates, $39 \%$ were masters students, and $8 \%$ were doctoral students. Only four of the participants had university meal plans. Hemoglobin A1C was taken only at baseline and the average among the thirty-six participants was 4.96 (SD $0.32 \%)$. There were no statistically significant differences in sex, BMI, student status, or Appalachian origin among the three groups at baseline. However, the FRUVED+LRC group was younger than the FRUVED+LF group (FRUVED+LRC 21.5 years versus FRUVED+LF 24.3 years, $p=0.01$ ) and the FRUVED group had a higher percentage of Caucasian individuals $(\mathrm{p}<0.05)$.

A decision was made to exclude 6 participants from analysis of metabolic parameters because of non compliance due to the following reasons: an average score less than 75 on the diet compliance continuum by the RDN over the last 6 weeks of the intervention, use of restricted medications, or changes in physical activity (Figure 2). There were no demographic differences between those completing all 8 weeks of the intervention $(n=36)$ and those included in the metabolic parameter analyses $(n=30$ (Table 2). 


\section{Intervention Effects on Food Consumption}

Dietary behavior changes over time in the 36 individuals are presented in Table 3.

Overall, the tailored intervention resulted in an increase in percentage of calories coming from carbohydrate and protein $(\mathrm{p}=0.0006$ and $\mathrm{p}=0.009$ respectively $)$, grams of fiber $(\mathrm{p}<0.0001)$, grams of total sugar $(\mathrm{p}=0.03)$, and total cups of fruit and vegetables $(\mathrm{p}<0.0001)$ (Figure 3 ). Fruit and vegetables were averaging close to recommendations ( 4.5 cups per day) at week 8 along with consumption of total fiber (25 grams per day) [7]. Fiber was statistically significant among groups $(\mathrm{p}=0.04)$; the FRUVED + LRC had lower fiber than the FRUVED + LF group. In addition, significant decreases were seen in percentage of calories coming from fat $(\mathrm{p}=0.02)$ and empty calories ( $p<0.0001)$ (Figure 4) after 8 weeks. There were no significant changes in total calories or breakdown of fats. Significant increases in insoluble fiber (non-fermentable, adds bulk to stool) (Figure 5) and soluble fiber (fermentable, water attractant) (Figure 6) were seen after the intervention (both $\mathrm{p}<0.0001)$ and among groups $(\mathrm{p}=0.0003, \mathrm{p}<0.0001$ respectively). There were no significant group by time interaction differences in the measured dietary variables, suggesting that three dietary groups followed a similar dietary patterns across 8 weeks, specifically in macronutrient breakdown.

\section{Intervention Effects on Other Behaviors}

Table 4 lists the self-reported data of behaviors among the 30 complaint individuals measured before and after the intervention that could influence metabolic parameters. Hours of sleep, number of steps, or stress from baseline to post intervention were maintained as requested, although there was a trend in a decrease in the number of steps $(\mathrm{p}=0.05)$ from baseline to post intervention. 


\section{Intervention Effects on Metabolic Parameters}

The dietary intervention, using tailored and personalized dietary counseling, significantly decreased waist circumference $(\mathrm{p}<0.0001)$, neck circumference $(\mathrm{p}=0.01)$, systolic blood pressure $(\mathrm{SBP})(\mathrm{p}=0.004)$, and high density lipoprotein $(\mathrm{HDL})(\mathrm{p}=0.0005)($ Table 5) in the 30 complaint individuals. Ratios of total cholesterol to HDL cholesterol and low-density lipoprotein (LDL) cholesterol to HDL cholesterol significantly increased $(p=0.006$ and $p=0.002$ respectively) following the intervention. The dietary intervention also resulted in significant increases in potassium levels $(\mathrm{p}=0.006)$. There were no significant changes in, sodium, glucose, insulin, HOMA-IR, calculated LDL cholesterol, triglycerides (TG), or hs-CRP after the 8 weeks or among groups. However, weight, BMI, and total cholesterol were approaching significant reductions over time $(\mathrm{p}=0.07, \mathrm{p}=0.05$, and $\mathrm{p}=0.05$ respectively).

\section{Discussion}

This randomized trial demonstrated the feasibility of delivering a tailored, dietary intervention to young adults attending WVU and the decrease in metabolic parameters after adopting the national nutrition guidelines. By tailoring the 2010 Dietary Guidelines for Americans to the individuals of this study, the researchers were effective in reducing calories from saturated fat and added sugars, while adding calories from fiber, fruits, and vegetables that averaged above recommendations. To our knowledge, limited randomized trials have been conducted to evaluate the impact of a dietary intervention using the Social Cognitive Theory (SCT) and a tailored intervention among young adults attending a university in the Appalachian region. This study showed a positive effect on dietary changes for free-living individuals outside of a controlled laboratory setting, which increases the likelihood that these results may be generalizable to "real-world" situations. Differences were not observed among the three dietary 
arms, therefore the addition of a lower fat or lower carbohydrate diet pattern did not appear to be the causal element of the improved metabolic parameters.

These results are similar to other studies that have demonstrated effects on food consumption using a personalized approach. For instance, researchers of the Puget Sound Eating Pattern study, which was a tailored, multi-component, self-help intervention in over 1400 adults (18-69 years old), promoted increased fruit and vegetable intake and lower calories from fat [117]. This study was similar to ours in that they used personalized feedback and supplementary tools such as a package of self-help materials and a dietary analysis with behavioral feedback. Moreover, Wright and colleagues found that using printed, tailored, iterative dietary feedback was more effective in improving short-term (3 months) fruit intake compared to group nutrition education consisting of two 90-minute, dietitian-led sessions [38]. In comparison, Brinberg et al. used tailored sessions with a nutritionist in college students and saw increased food choices that were high in fiber [118]. Our study utilized both individual and group level education that provided the foundational knowledge to change the behavior and utilized personal data to educate individuals to change their behavior. This combined approach appeared to be effective in changing nutrition behavior among a population undergoing significant transformations in their lifestyle.

Comparing the results of our study to other nutritional intervention studies using behavioral theory, Ha and Caine-Bish evaluated the effectiveness of a 15-week basic nutrition course in increasing intake of fruit and vegetables among college students [99]. The researchers guided their lectures and activities around the principles of the SCT and results indicated statistically significant improved consumption of fruit and vegetables at the end of the course. Furthermore, Cox et al. assessed the effectiveness of SCT constructs to explain dietary outcomes 
of a sample of university students. The researchers reported four practical implications for interventions in this population: enhance self-efficacy for healthy eating, increase their access to healthy food, decrease the availability of foods to limit, and enhance their behavioral skills for preparing certain foods [45]. Although our study provided tips on the access and availability of foods, as well as gave dining hall tours to a couple of participants, this was the primary focus of the bigger project 'Get FRUVED', that advertised health messages across campus and sponsored well-being events for students to attend in which our participants may have been exposed to. However, our study utilized the components of self-efficacy and skill building of the SCT with group education and one-on-one counseling sessions with the RDN. Thus the results of the previous studies and our study indicate the importance of designing interventions guided by theoretical framework of the SCT to increase consumption of healthy food.

As a result of adopting the nutritional guidelines, the participants who complied with our study intervention decreased systolic blood pressure (SBP). Researchers have reported that university students who consumed higher amounts of fruits and vegetables $(726 \mathrm{~g}$ of fruit and 957g of vegetables) had lower SBP (2 mmHg) compared to those who consumed the lowest amount of fruits and vegetables (114g of fruits and 240g of vegetables) [8] . Our results show clinical significance given that research has linked high levels of SBP to CVD $[119,120]$ and when SBP is reduced by $10 \mathrm{mmHg}$, CVD events are decreased by $41 \%$ [121]. A study in adults that investigated the effects of the Dietary Approaches to Stop Hypertension (DASH) on metabolic parameters of the metabolic syndrome (MetS) showed that after 6 months, the participants decreased their SBP $(-12$ and $-11 \mathrm{mmHg})$ and DBP $(-6$ and $-7 \mathrm{mmHg})$ in men and women respectively. We report a reduction in average SBP of almost $7 \mathrm{mmHg}$ in 8 weeks utilizing the Dietary Guidelines for Americans. Decreases in SBP may be caused by the increase 
in potassium levels of our subjects, where studies reported that supplementation with potassium was associated with a significant reduction in mean SBP of $3.11 \mathrm{~mm} \mathrm{Hg}$ [122]. Therefore diets that contains high levels of potassium-rich foods (i.e. fruit and vegetables) may help regulate blood pressure.

In addition to blood pressure, we observed significant reductions in waist and neck circumference in the compliant individuals. These two indices are measures of upper-body obesity and have been associated with cardiometabolic disease [123, 124]. Additionally, total weight and BMI were approaching significant decreases where we observed an average of $1 \%$ (2.2 pounds) weight loss after 8 weeks. Modest weight loss of 5-10\% in adults 45-76 years has been associated with significant improvements in CVD risk factor at one year [125]. Although there were no significant changes in total caloric consumption, the average decrease in 350 calories daily from baseline to post in our participants is noteworthy given that if this is sustained for one year, our individuals could lose an estimated 26 pounds or $15 \%$ weight loss. More importantly, these changes in metabolic parameters and upper-body obesity were primarily due to changes in dietary behaviors because no other behavioral influences (i.e. sleep, physical activity or stress) were changed. However, these behaviors were only measured at baseline and post intervention and some were self-reported. Yet the researchers did their best to control these influences by weekly interaction with the dietitian and having the participant report activities on their logs.

Although most of the outcomes we found were favorable to health, including a trend in lowered total cholesterol (TC), our intervention resulted in decreases in HDL cholesterol, where higher levels of HDL have been reported as a protective effect in CVD [126]. Similarly, studies have reported lowered HDL levels after participants underwent a high fiber diet [127, 128]. We 
also found increases in the ratios of TC/HDL and LDL/HDL cholesterol that are also linked to CVD $[129,130]$. This unfavorable effect could be due in part to the reduced fat content of the diet and an increased proportion of fiber, where a meta-analysis found that replacement of fat with carbohydrates will increase the ratio of total cholesterol to HDL cholesterol [131]. To influence the lipoprotein ratios and increase HDL cholesterol, researchers report that changing the proportions of dietary fatty acids (i.e. replacing trans fatty acids with unsaturated fatty acids) instead of reducing total fat intake is more favorable [132]. This may be the reason why we observed such changes in lipoprotein measures because our intervention was designed to reduce calories from fat instead of replace with healthier alternatives (i.e. polyunsaturated fat or monounsaturated fat). Nonetheless, our intervention produced decreases in TC by $9.3 \mathrm{mg} / \mathrm{dL}(0.5$ $\mathrm{mmol} / \mathrm{l})$, where decreases in $0.6 \mathrm{mmol} / \mathrm{l}$ in men at age 40 decreased ischemic heart disease of $54 \%[133]$

Most of the outcomes of our study are promising for health and we speculate that the reason for these changes occurred because we chose to focus on high-risk individuals who have poor lifestyles behaviors, live in a region with high magnitude of disease, and are at a transitional time of increased risk of developing obesity [25]. Several studies have shown positive dietary changes in using assumed healthy population with no identification of risk factors. A study in the Netherlands used group intervention sessions and tailored letters to increase consumption of fruits and vegetables [134] among individuals with three risk factors for cardiovascular disease. The Dean Ornish Program has been successful in reducing fat intake and other coronary events in those with coronary artery disease [135]. Similar to our target population of young adults, an intervention using technology to prevent weight gain in "at-risk" students attending a 2-year community college in Minnesota resulted in significant decreases in fast-food consumption after 
4 months [136]. One underlying idea of studying "at-risk" individuals is that the individual becomes knowledgeable of their risk and can make informed decisions about modification of lifestyle habits on their own [137]. We speculate that our individuals took advantage of the intervention due to knowing their risk of weight gain or disease would occur if they continued with usual behaviors. All-in-all, it may be beneficial to target individuals with elevated disease risk for dietary behavior change [33].

A few limitations of our study include a short intervention period and a modest sample size that limited the power to detect dietary changes across the three intervention arms and observe additional changes in clinical parameters (i.e. LDL cholesterol, body fat percentage, weight). In looking at other dietary studies, others were able to elicit metabolic effects in as little as 6 weeks [138] and as long as 2 years [17]. Given the feasibility of such an intense intervention using personalized counseling, 8 weeks was chosen for the FRUVEDomics pilot study. However it is important to highlight that we had low attrition rates due to regular weekly meetings with the RDN, periodic incentive distributions, and targeting young adults in the "preparation" stage of change at baseline. The weekly counseling sessions also made it possible to accurately assess dietary intake to make conclusions about lack of compliance for study participants. Yet the feasibility of weekly counseling sessions with a RDN might not be appropriate for a campuswide setting, therefore implementation of mobile applications could be a useful alternative in producing behavior change among larger cohorts [139] or utilizing existing personnel on campus who can deliver weekly nutritional message to students (i.e. Residential Assistant). Although our sample size had little statistical power to compare across diet interventions $(\beta=0.75$ with $\alpha<0.05$ using $n=10$ /group), a strength of our study design was that all the subjects served as their own controls and the effect within any specific diet had more than adequate power $(>0.95$, using $\alpha$ 
$<0.05)$. Moreover, our spread of ethnicity was small and reflects the demographics of young adults in West Virginia, thus making the study not generalizable to all young adults. Lastly, at the time of the intervention, the USDA 2010 were the published guidelines to conduct our intervention. Future research would benefit from utilizing the 2015 DGAs to investigate dietary behavior changes and metabolic outcomes in young adults.

\section{Conclusion}

This study design is encouraging for young adults attending a university who may exhibit poor lifestyle behavior choices that can increase disease risk. The FRUVEDomics pilot study was centered on improving dietary behaviors using the population based recommendations by the USDA and grounded in theoretical framework. The intervention resulted in increased fruit and vegetable and lowered fat intake, which improved waist circumference and systolic blood pressure in 8 weeks.

Although dietary manipulation is used as a strategy to reduce metabolic diseases in the short-term, intervention trials for young adults attending college are lacking. With obesity and cardiovascular risk increasing among younger generations of developing countries, more studies using primary prevention methods, grounded in theory are needed. The data presented here provides preliminary evidence that a tailored intervention, using nutrition focused prevention, theoretical foundation, and national guidelines, may be effective in producing individual changes associated with disease outcomes. 


\section{Tables/Figures}

Table 1: Dietary Arms in FRUVEDomics Study

$\begin{array}{|ll|}\text { Diet } & \text { Main Components } \\ \text { FRUVED } & \begin{array}{l}50 \% \text { of plate fruit and vegetables; equivalent to } 2.5 \text { cups vegetables for women and } 3 \text { cups for men daily. } \\ \text { Fruit intake was } 2 \text { cups daily for all individuals. }\end{array} \\ \text { FRUVED+LRC } & \begin{array}{l}\text { FRUVED diet plus choose low-glycemic foods (particularly non-starchy vegetables, legumes, and temperate } \\ \text { fruits) and limit intake of high-glycemic foods (i.e. refined grains, starchy vegetables, fruit juices, and } \\ \text { sweets). }\end{array} \\ \text { FRUVED+LF } & \begin{array}{l}\text { FRUVED diet plus 30\% of their calories from fat, with only 10\% or less coming from saturated fat, and } 300 \\ \text { mg of cholesterol per day. Participants were told to make most of their protein and dairy sources lean. }\end{array}\end{array}$

FRUVED (High Fruit and Vegetables) diet was set by national standards from the Center for Disease Control and Prevention based on the MyPlate recommendations [7]. The FRUVED+LRC (High Fruit and Vegetables, low refined carbohydrate) diet has standards published elsewhere [21] and the FRUVED+LF (High fruit and vegetables, low fat) diet was based off the American Heart Association guidelines [6]. 
Table 2: Demographic and Health Related Characteristics at Baseline

\begin{tabular}{|c|c|c|c|c|c|}
\hline & $\begin{array}{c}\text { Total } \\
(\mathrm{n}=36)\end{array}$ & $\begin{array}{l}\text { Included }^{\mathrm{a}} \\
\quad(\mathrm{n}=30)\end{array}$ & $\begin{array}{l}\text { Fruved } \\
(\mathrm{n}=12)\end{array}$ & $\begin{array}{c}\text { Fruved + LFC } \\
(n=12)\end{array}$ & $\begin{array}{l}\text { Fruved + LF } \\
\quad(n=12)\end{array}$ \\
\hline Age $(x \pm S D)$ & $23.17 \pm 2.50$ & $23.63 \pm 2.36$ & $23.67 \pm 1.50$ & $21.50 \pm 2.91 * *$ & $24.33 \pm 2.10$ \\
\hline Sex (\% male) & 42 & 47 & 42 & 42 & 42 \\
\hline $\begin{array}{l}\text { Race/ethnicity (\%) } \\
\text { White } \\
\text { African-American } \\
\text { Asian } \\
\text { Hispanic } \\
\text { Other }\end{array}$ & $\begin{array}{c}61 \\
11 \\
11 \\
11 \\
6\end{array}$ & $\begin{array}{r}67 \\
7 \\
10 \\
10 \\
6\end{array}$ & $\begin{array}{l}84 * * \\
0 * * \\
0 * * \\
8 \\
8\end{array}$ & $\begin{array}{l}41 \\
17 \\
17 \\
25^{* *} \\
0\end{array}$ & $\begin{array}{r}58.3 \\
16.7 \\
8.3 \\
8.3 \\
8.3\end{array}$ \\
\hline $\begin{array}{l}\text { Student Status (\%) } \\
\text { Undergraduate } \\
\text { Masters } \\
\text { PhD } \\
\text { None Student }\end{array}$ & $\begin{array}{c}45 \\
39 \\
8 \\
8\end{array}$ & $\begin{array}{l}33 \\
47 \\
10 \\
10\end{array}$ & $\begin{array}{c}25 \\
59 \\
8 \\
8\end{array}$ & $\begin{array}{c}76 \\
8 \\
8 \\
8\end{array}$ & $\begin{array}{c}34 \\
50 \\
8 \\
8\end{array}$ \\
\hline $\begin{array}{l}\text { Body Mass Index (x } \pm \\
\text { SD) }\end{array}$ & $27.04 \pm 6.41$ & $25.99 \pm 4.17$ & $27.39 \pm 5.04$ & $26.54 \pm 8.06$ & $27.18 \pm 6.33$ \\
\hline $\begin{array}{l}\text { Hemoglobin A1C (\%) } \\
(\mathrm{x} \pm \mathrm{SD})\end{array}$ & $4.96 \pm 0.32$ & $4.89 \pm 0.32$ & $4.94 \pm 0.32$ & $4.89 \pm 0.31$ & $4.94 \pm 0.35$ \\
\hline From Appalachia (\%) & 53 & 60 & $75 * *$ & 42 & 41.7 \\
\hline
\end{tabular}

${ }^{a}$ Individuals included in analysis of metabolic parameters based on high compliance score from RDN and without changes in physical activity, stress and sleep over the intervention.

$* \mathrm{P}<0.05$ between total and included participants

$* * \mathrm{P}<0.05$ among intervention groups 
Table 3: Treatment Effects on Dietary Factors across the Duration of the Study

\begin{tabular}{|c|c|c|c|c|c|}
\hline & \multicolumn{2}{|c|}{ All subjects $(\mathrm{N}=30)$} & Group Effect & Time Effect & Group*Time Effect \\
\hline & Pre & Post & P-Value & P-Value & P-Value \\
\hline Total Calories, Kcal/d & $2186.0 \pm 1294.6$ & $1837.0 \pm 617.0$ & 0.86 & 0.24 & 0.43 \\
\hline Empty Calories ${ }^{2}, \mathrm{Kcal} / \mathrm{d}$ & $967.0 \pm 855.7$ & $420.7 \pm 233.9$ & 0.57 & $<0.0001 *$ & 0.58 \\
\hline Fruit and vegetables, cups/d & $2.3 \pm 1.9$ & $4.9 \pm 2.2$ & 0.27 & $<0.0001^{*}$ & 0.79 \\
\hline Total Carbohydrates, $\%$ & $43.0 \pm 13.0$ & $51.9 \pm 10.5$ & 0.10 & $0.0006^{*}$ & 0.41 \\
\hline Total fiber, $\mathrm{g}$ & $18.1 \pm 11.9$ & $31.4 \pm 15.2$ & $0.04 *$ & $<0.0001^{*}$ & 093 \\
\hline Insoluble Fiber, g & $1.3 \pm 2.0$ & $3.1 \pm 2.4$ & $0.0003 *$ & $<0.0001 *$ & 0.66 \\
\hline Soluble Fiber, g & $0.2 \pm 0.4$ & $0.7 \pm 0.6$ & $<0.0001^{*}$ & $<0.0001^{*}$ & 0.59 \\
\hline Total sugar, g & $76.9 \pm 32.7$ & $95.2 \pm 46.7$ & 0.41 & $0.03 *$ & 0.61 \\
\hline Total Protein, $\%$ & $16.0 \pm 6.0$ & $19.1 \pm 4.4$ & 0.62 & $0.009^{*}$ & 0.44 \\
\hline Total Fat, \% & $36.0 \pm 10.4$ & $31.5 \pm 10.6$ & 0.14 & $0.02 *$ & 0.47 \\
\hline Saturated fat, $g$ & $27.3 \pm 20.3$ & $19.9 \pm 12.6$ & 0.66 & 0.13 & 0.16 \\
\hline Monounsaturated fat, $g$ & $19.7 \pm 21.5$ & $16.7 \pm 11.3$ & 0.80 & 0.92 & 0.26 \\
\hline Polyunsaturated fat, $g$ & $215.5 \pm 202.4$ & $236.2 \pm 233.8$ & 0.21 & 0.86 & 0.55 \\
\hline Cholesterol, mg & $215.5 \pm 202.4$ & $236.2 \pm 233.8$ & 0.21 & 0.86 & 0.55 \\
\hline
\end{tabular}

The intervention groups (FRUVED, FRUVED+LRC, FRUVED+LF) were followed weekly and dietary factors were collected and analyzed for main effects of group, time, and their interaction using Repeated Measures ANOVA. Significant effects ANOVA were followed by multiple comparisons using Tukey adjustment. Untransformed baseline and post intervention means and standard deviations are listed, along with p-values for the main effects and interaction.

$* \mathrm{P}<0.05$ 
Table 4: Treatment effects on Physical Activity, Stress and Sleep on Compliant Individuals $(\mathrm{N}=30)^{\mathrm{a}}$

\begin{tabular}{|l|c|c|c|c|c|}
\hline Domain & $\begin{array}{c}\text { Pre Intervention } \\
(\text { Mean } \pm \text { SD })\end{array}$ & $\begin{array}{c}\text { Post Intervention } \\
(\text { Mean } \pm \text { SD) }\end{array}$ & $\begin{array}{c}\text { Group Effect } \\
(\mathrm{p} \text {-value })\end{array}$ & $\begin{array}{c}\text { Time Effect } \\
(\mathrm{p} \text {-value })\end{array}$ & $\begin{array}{c}\text { Group*Time Effect } \\
(\mathrm{p} \text {-value })\end{array}$ \\
\hline $\begin{array}{l}\text { Self-Reported Hours of } \\
\text { Sleep }\end{array}$ & $7.24 \pm 1.11$ & $7.08 \pm 0.92$ & 0.69 & 0.29 & 0.12 \\
\hline $\begin{array}{l}\text { ActiGraph accelerometer - } \\
\text { Steps }\end{array}$ & $49,921.77 \pm 16553.4$ & $44,104.48 \pm 17,299.67$ & 0.18 & 0.05 & 0.66 \\
\hline $\begin{array}{l}\text { Cohen's Perceived Stress } \\
\text { Score }\end{array}$ & $24.53 \pm 9.24$ & $25.21 \pm 7.63$ & 0.46 & 0.78 & 0.72 \\
\hline
\end{tabular}

Self-reported behavioral measurements were collected from all individuals at baseline and post intervention via survey. Repeated Measures ANOVA was used to analyze the main effects of group (FRUVED n=12, FRUVED+LRC n=8, FRUVED+LF n=10), time, and their interaction. Significant effects ANOVA were followed by multiple comparisons using Tukey adjustment. Untransformed baseline and post intervention means and standard deviations are listed, along with p-values for the main effects and interaction.

${ }^{a}$ Individuals included in analysis based on high compliance score from RDN and without changes in physical activity, stress and sleep over the intervention.

$* \mathrm{P}<0.05$

${ }^{1}$ The question taken from the Pittsburgh Sleep Quality Index read, "How many hours of actual sleep did you get at night?". 
Table 5: Treatment Effects on Clinical Measures at Pre and Post Intervention on Compliant Individuals $(\mathrm{N}=30)^{\mathrm{a}}$

\begin{tabular}{|c|c|c|c|c|c|}
\hline Domain & $\begin{array}{c}\text { Pre Intervention } \\
(\text { Mean } \pm \text { SD) }\end{array}$ & $\begin{array}{l}\text { Post Intervention } \\
\quad(\text { Mean } \pm \text { SD) }\end{array}$ & $\begin{array}{l}\text { Group Effect } \\
\text { (p-value) }\end{array}$ & $\begin{array}{c}\text { Time Effect } \\
\text { (p-value) }\end{array}$ & $\begin{array}{c}\text { Group*Time Effect } \\
\text { (p-value) }\end{array}$ \\
\hline Weight $(\mathrm{kg})$ & $77.0 \pm 15.1$ & $76.0 \pm 14.5$ & 0.49 & 0.07 & 0.48 \\
\hline $\mathrm{BMI}\left(\mathrm{kg} / \mathrm{m}^{2}\right)$ & $26.4 \pm 4.2$ & $26.0 \pm 4.2$ & 0.33 & 0.05 & 0.88 \\
\hline Waist Circumference $(\mathrm{cm})$ & $83.4 \pm 11.7$ & $80.6 \pm 10.4$ & 0.76 & $<0.0001 *$ & 0.54 \\
\hline Hip Circumference $(\mathrm{cm})$ & $102.9 \pm 9.8$ & $102.2 \pm 8.8$ & 0.36 & 0.14 & 0.36 \\
\hline Neck Circumference $(\mathrm{cm})$ & $36.0 \pm 3.9$ & $35.6 \pm 3.5$ & 0.97 & $0.01 *$ & 0.47 \\
\hline Systolic Blood Pressure (mmHg) & $118.6 \pm 15.3$ & $111.8 \pm 9.7$ & 0.39 & $0.004 *$ & 0.57 \\
\hline Diastolic Blood Pressure (mmHg) & $62.7 \pm 9.3$ & $60.3 \pm 7.2$ & 0.36 & 0.12 & 0.76 \\
\hline Body Fat $(\%)$ & $28.6 \pm 14.3$ & $25.9 \pm 10.7$ & 0.60 & 0.14 & 0.37 \\
\hline Sodium $(\mathrm{mmol} / \mathrm{L})$ & $139.1 \pm 1.5$ & $138.2 \pm 3.2$ & 0.30 & 0.10 & 0.57 \\
\hline Potassium (mmol/L) & $3.8 \pm 0.3$ & $4.0 \pm 0.2$ & 0.72 & $0.006^{*}$ & 0.86 \\
\hline Glucose (mg/dL) & $86.9 \pm 7.5$ & $87.1 \pm 9.3$ & 0.29 & 0.87 & 0.17 \\
\hline Insulin (uLU/mL) & $7.0 \pm 3.3$ & $7.4 \pm 4.1$ & 0.18 & 0.82 & 0.12 \\
\hline HOMA IR & $1.5 \pm 0.7$ & $1.6 \pm 0.9$ & 0.10 & 0.82 & 0.08 \\
\hline Total Cholesterol (mg/dL) & $181.0 \pm 29.1$ & $171.7 \pm 29.3$ & 0.55 & 0.05 & 0.67 \\
\hline High Density Lipoprotein (mg/dL) & $56.6 \pm 12.4$ & $50.3 \pm 10.4$ & 0.96 & $0.0005 *$ & 0.50 \\
\hline Low Density Lipoprotein (mg/dL) & $105.7 \pm 22.9$ & $103.7 \pm 23.9$ & 0.42 & 0.66 & 0.41 \\
\hline TC/HDL (mg/dL) & $3.3 \pm 0.8$ & $3.5 \pm 0.7$ & 0.81 & $0.006^{*}$ & 0.76 \\
\hline LDL/HDL (mg/dL) & $2.0 \pm 0.7$ & $2.1 \pm 0.6$ & 0.70 & $0.002 *$ & 0.63 \\
\hline Triglycerides (mg/dL) & $92.7 \pm 31.9$ & $87.9 \pm 30.4$ & 0.77 & 0.24 & 0.23 \\
\hline hs-CRP (mg/dL) & $0.3 \pm 0.5$ & $0.5 \pm 0.4$ & 0.07 & 0.05 & 0.59 \\
\hline
\end{tabular}

Biochemical and anthropometric measures were collected from all individuals at baseline and post intervention. Repeated Measures ANOVA was used to analyze the main effects of group (FRUVED n=12, FRUVED+LRC $n=8$, FRUVED+LF n=10), time, and their interaction. Significant effects ANOVA were followed by multiple comparisons using Tukey adjustment. Untransformed baseline and post intervention means and standard deviations are listed, along with p-values for the main effects and interaction.

TC-Total Cholesterol; HDL-High Density Lipoprotein; LDL-Low Density Lipoprotein; high-sensitivity C-reactive protein

${ }^{a}$ Individuals included in analysis based on high compliance score from RDN and without changes in physical activity, stress and sleep over the intervention.

$* \mathrm{P}<0.05$ 


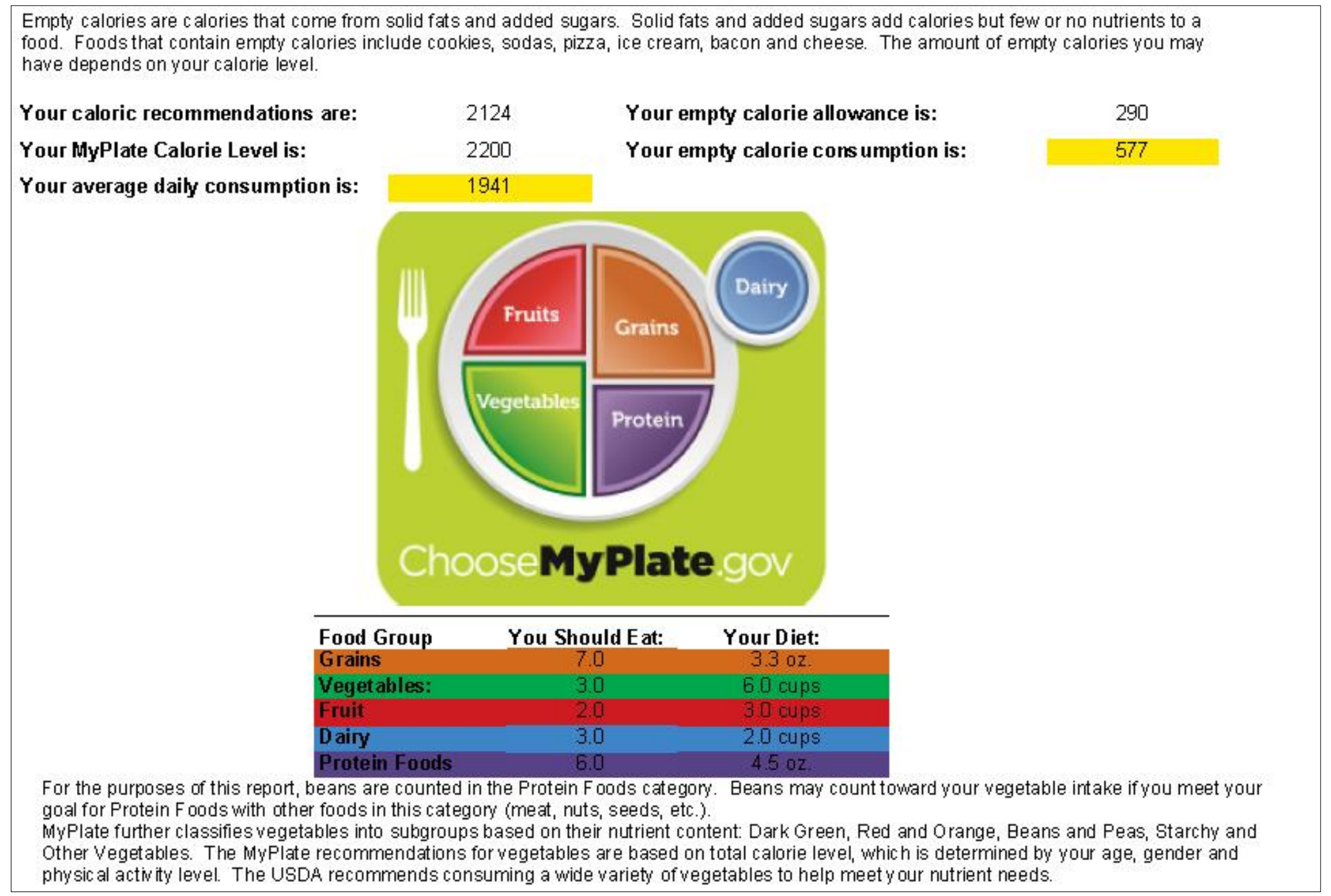

Figure 1. Nutritionist Pro $^{\mathrm{TM}}$ analysis document used for individual counseling 


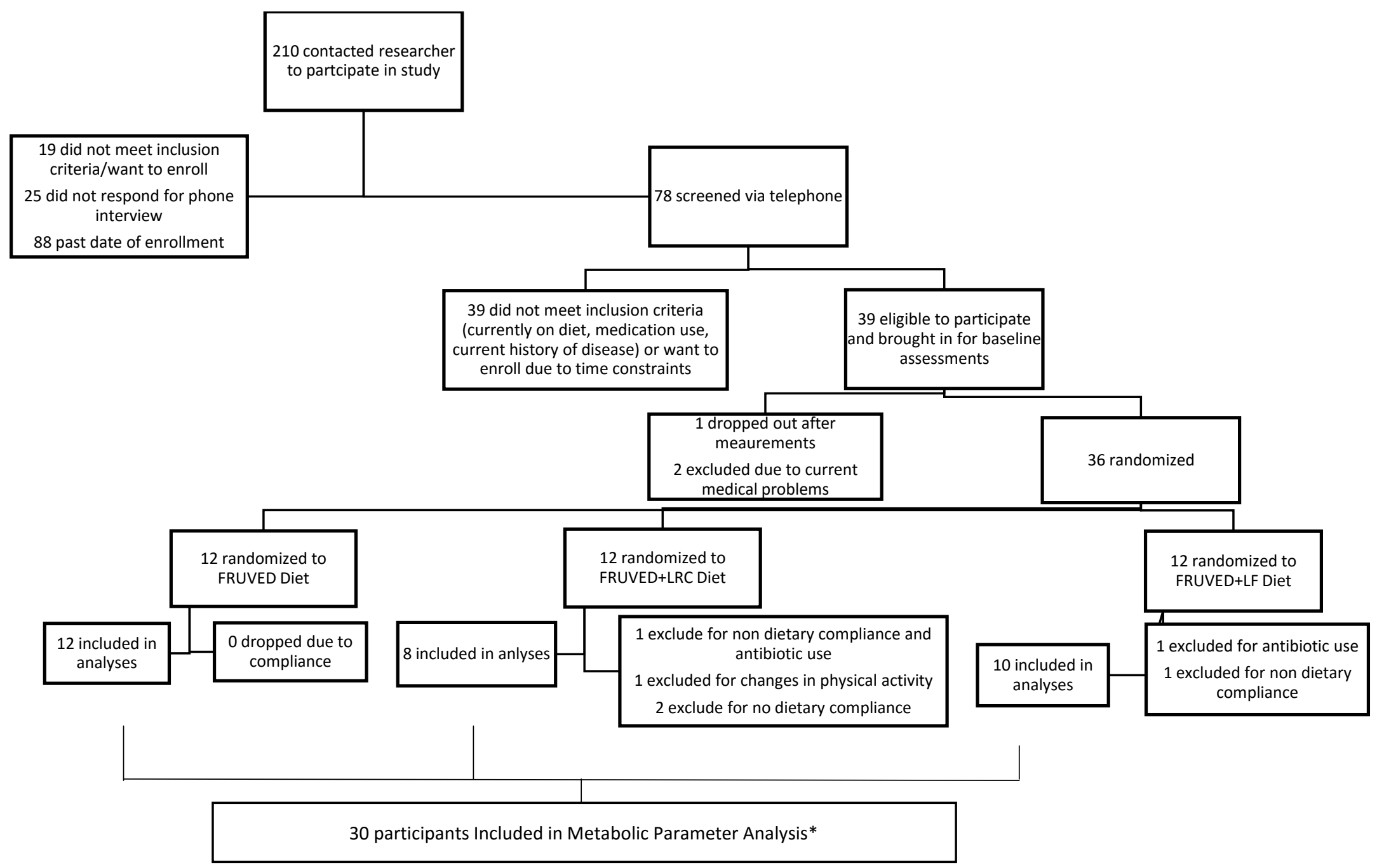

Figure 2. Flow of participants through the trial

*Individuals included in analysis for changes in metabolic parameters based on high compliance nutrition score from RDN, no use of restricted medications, and without changes in physical activity, stress and sleep over the 8 week intervention. 


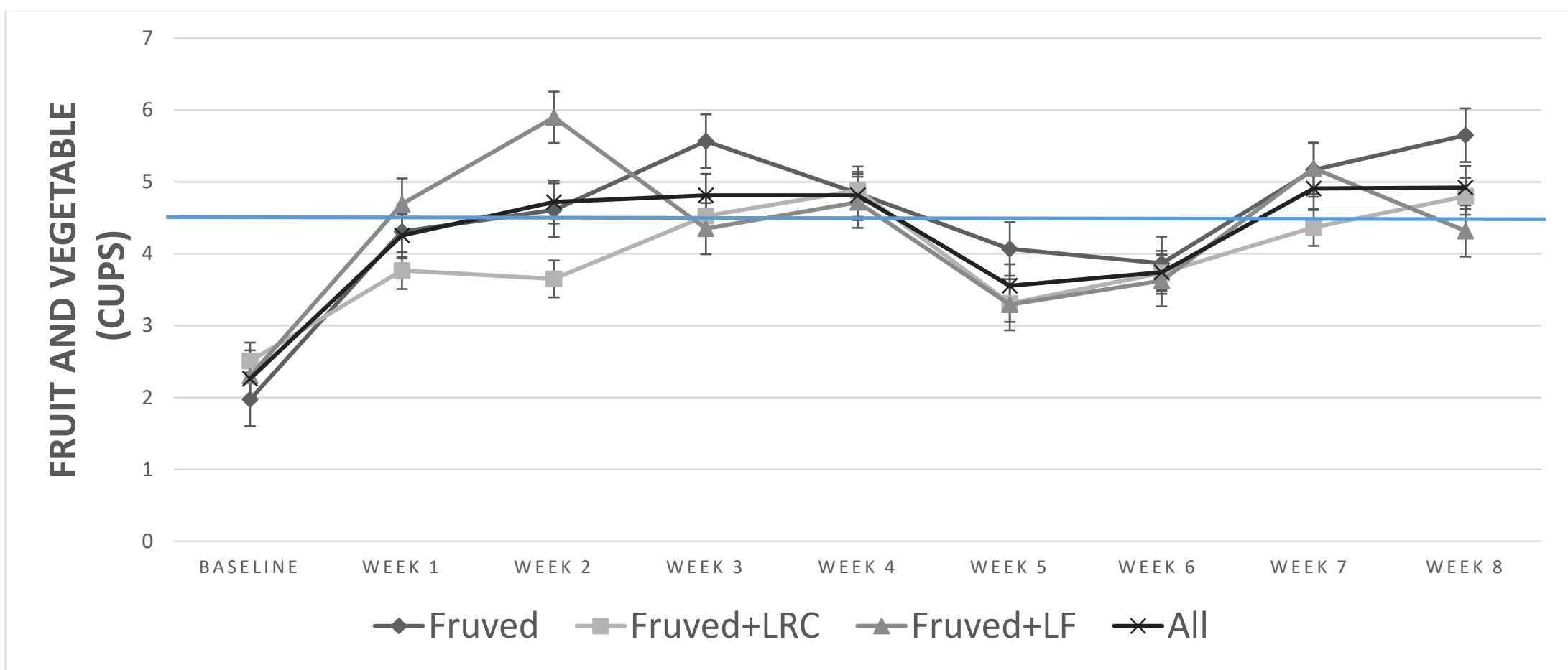

Figure 3. Fruit and vegetable consumption (cups) across time. The intervention groups (FRUVED, FRUVED+LRC, FRUVED+LF) were followed weekly and dietary factors were collected and analyzed for main effects of group, time, and their interaction using repeated measures ANOVA. Significant effects ANOVA were followed by multiple comparisons using Tukey adjustment. Significant effect of time (intervention) was observed for number of cups of fruits and vegetables $(p<0.0001)$.

— Recommended amount of fruit ( 2 cups) and vegetables ( $21 \% 2$ cups) for a 2000 calorie diet by the USDA guidelines). 


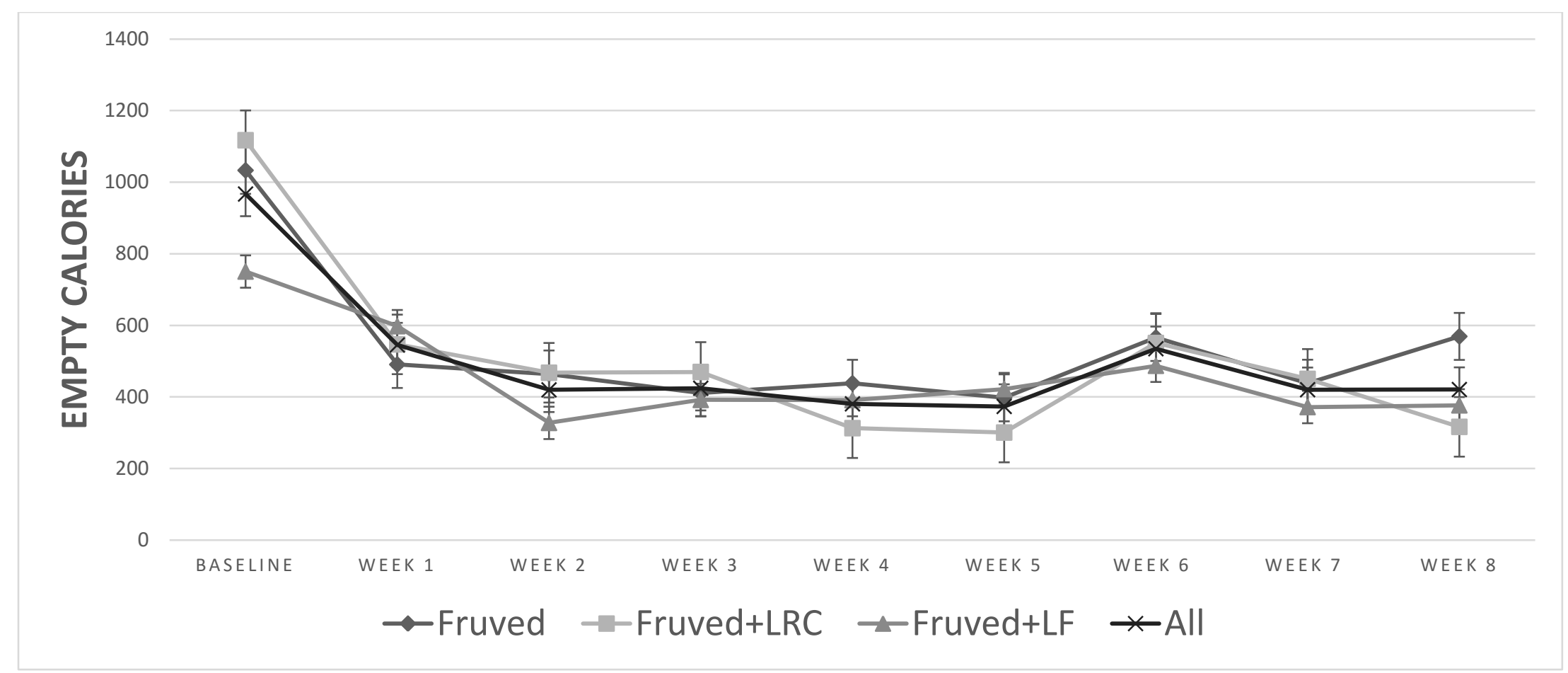

Figure 4: Empty calories (calories from solid fat and added sugars) consumption across time. The intervention groups (FRUVED, FRUVED+LRC, FRUVED+LF) were followed weekly and dietary factors were collected and analyzed for main effects of group, time, and their interaction using repeated measures ANOVA. Significant effects ANOVA were followed by multiple comparisons using Tukey adjustment. Significant effect of time (intervention) was observed for empty calories ( $p<0.0001$ ). 


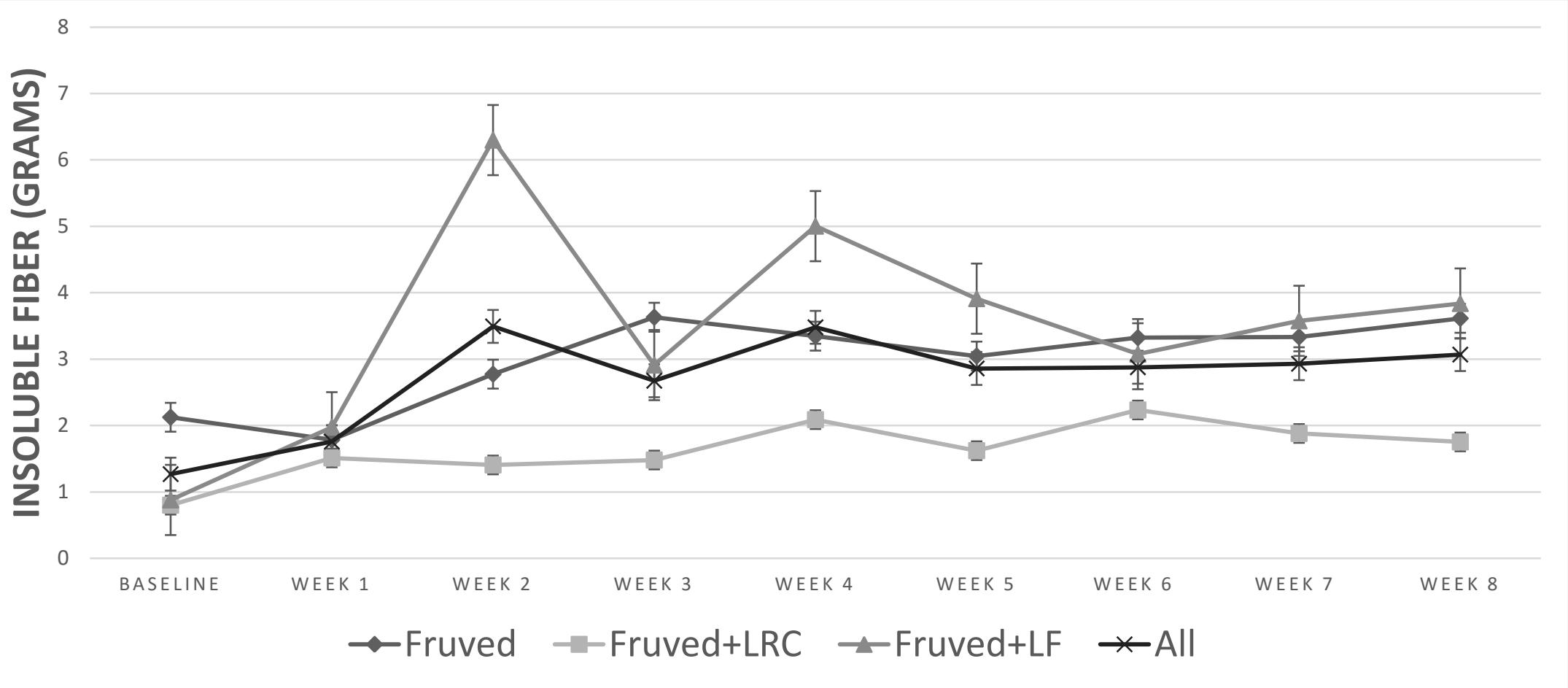

Figure 5: Insoluble fiber consumption (grams) across time. The intervention groups (FRUVED, FRUVED+LRC, FRUVED+LF) were followed weekly and dietary factors were collected and analyzed for main effects of group, time, and their interaction using repeated measures ANOVA. Significant effects ANOVA were followed by multiple comparisons using Tukey adjustment. Significant effect of intervention group as well as time were observed for insoluble fiber $(\mathrm{p}=0.0003$ and $\mathrm{p}=0.002$, respectively). 


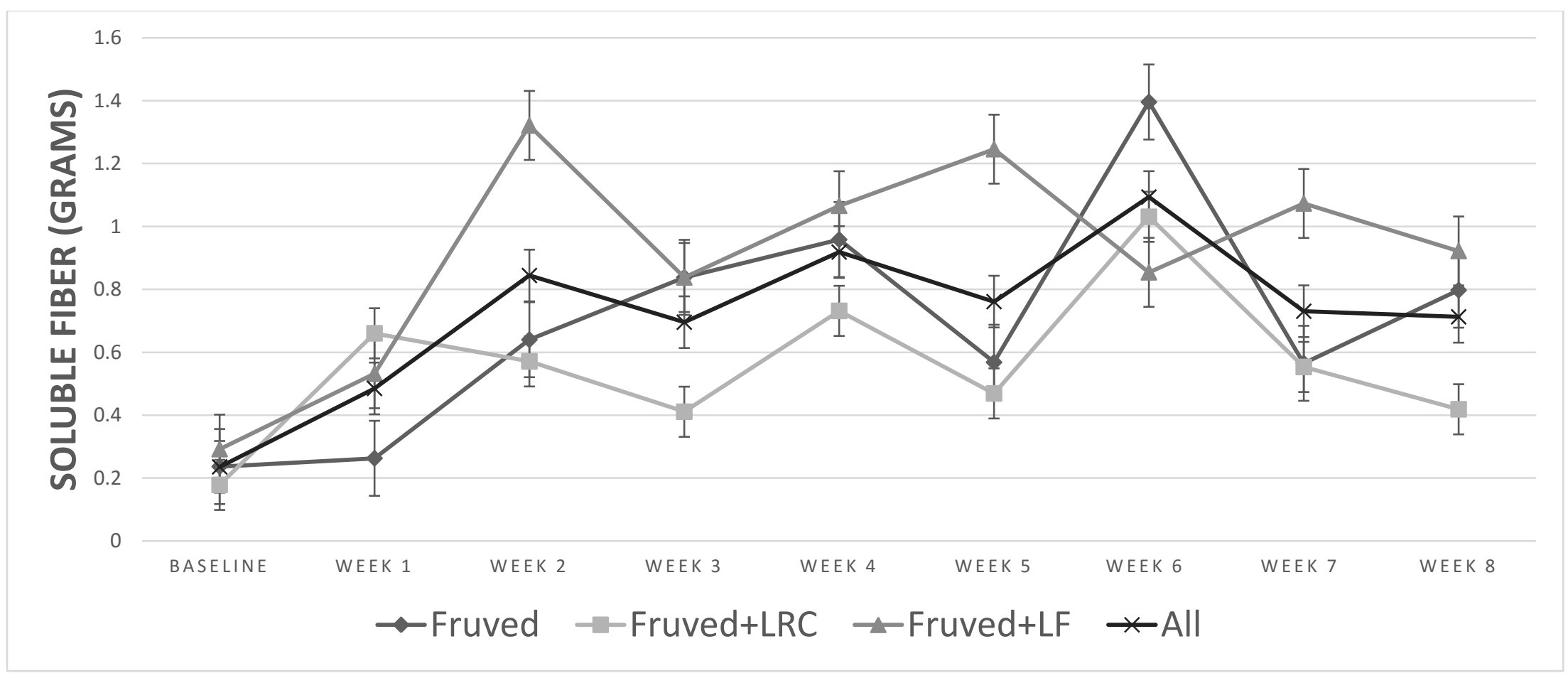

Figure 6: Soluble fiber consumption (grams) across time. The intervention groups (FRUVED, FRUVED+LRC, FRUVED+LF) were followed weekly and dietary factors were collected and analyzed for main effects of group, time, and their interaction using repeated measures ANOVA. Significant effects ANOVA were followed by multiple comparisons using Tukey adjustment. Significant effect of group and time were observed for soluble fiber $(\mathrm{p}=0.003$ and $\mathrm{p}<0.0001$, respectively). 
Chapter III: Impact of a Nutrition Intervention on the Microbiome of Young Adults 


\title{
Abstract
}

\section{Impact of a Nutrition Intervention on the Microbiome of Young Adults: FRUVEDomics Pilot Study}

\author{
${ }^{1}$ OA Famodu, ${ }^{1} \mathrm{MD}$ Olfert, ${ }^{1} \mathrm{ZC}$ Phipps, ${ }^{2} \mathrm{AF}$ Cockburn, ${ }^{2} \mathrm{AM}$ Infante,,${ }^{1}$ I Holásková, ${ }^{2} \mathrm{MT}$ \\ Downes, ${ }^{2} \mathrm{PJ}$ Murray, ${ }^{1} \mathrm{JW}$ McFadden, ${ }^{2} \mathrm{IM}$ Olfert, ${ }^{2} \mathrm{CF}$ Cuff \\ West Virginia University, ${ }^{1}$ Davis College Animal and Nutritional Sciences, and ${ }^{2}$ School of \\ Medicine Morgantown, WV
}

Background: Individuals have long since co-existed with their gut microbiota and it is now being accepted as indispensable in healthy living. Researchers have linked an imbalanced gut microbiome with obesity, atherosclerosis, and other chronic inflammatory diseases.

Objective: The purpose of this study was to examine the effects of a healthy dietary intervention on the microbiome of young adults and explore relationships with dietary and amino acid metabolites.

Methods: Subjects $(n=36)$ followed a free-living diet intervention for 8-weeks centered on the 2010 Dietary Guidelines for Americans and the Choose MyPlate recommendations. Venous blood samples and stool samples were collected at baseline and post intervention. Participants underwent individual weekly consultations with a Registered Dietitian Nutritionist, using food logs, food pictures and receipt management to evaluate adherence. Alterations in the fecal microbiome were assessed using high throughput sequencing of the V3-V4 regions of the $16 \mathrm{~S}$ rRNA gene and serum amino acids were profiled by targeted gas chromatography tandem mass spectrometry. Paired t-testing with inverse beta binomial distribution was used to assess changes in microbiome from baseline to post and linear regression was used to calculate relationships among microbiomes, diet variables, and serum amino acids.

Results: After 30 compliant individuals followed a healthy diet pattern (increased fruit and vegetables, increased fiber, and decreased calories from fat), there were significant changes to their microbiomes. The phylum of Firmicutes significantly decreased (fold change $=-1.3$, adj $\mathrm{p}=0.23$ ) along with the family Ruminococcaceae (fold change $=-1.3$, adj $\mathrm{p}=0.22$ ). A significant increase was seen in Bacteroidetes (fold change $=1.3$, adj $\mathrm{p}=0.23$ ), the family $\mathrm{S}-247$ (fold change $=4.0$, adj $\mathrm{p}<0.0001$ ), and the family of Bacteroidaceae (fold change=1.3, adj $\mathrm{p}<0.05)$. At baseline, Proteobacteria was positively related to cups of fruits and vegetables (estimate $=0.03, p$ $=0.01$ ) and the family Porphyromonadaceae was negatively related to grams of total sugar (estimate $=-0.001, \mathrm{p}=0.04$ ). Following the intervention, Verrucomicrobia was negatively related to alanine (estimate $=-0.0005, \mathrm{p}=0.03$ ) and Alcaligenaceae was positively related to insoluble fiber (estimate $=0.05, \mathrm{p}=0.02$ ).

Conclusion: Our findings indicate that a short-term, healthy diet changed the microbiota of young adults and these changes have been linked to improved metabolic parameters. Additionally, we found specific relationships with bacteria, dietary components, and serum amino acids. These relationships can lead to personalized nutrition methods for decreased progression of disease. 


\section{Introduction}

The rise in obesity and metabolic syndrome (MetS), a cluster of conditions that increases the risk of cardiovascular disease (CVD) and type II diabetes mellitus (T2DM), among younger generations [84] and the increased risk of developing MetS with aging [140] creates a need for primary prevention interventions for young adults. Primary treatment of MetS is lifestyle modification, such as control of blood glucose, blood pressure, dyslipidemia and obesity. In the INTERHEART study, researchers identified nine measurable and modifiable risk factors for disease prevention: smoking, hypertension, diabetes, abdominal obesity, increased alcohol, decreased physical activity, psychosocial factors, abnormal lipids, and low consumption of fruit and vegetables [141].

According to the World Health Organization (WHO), it is estimated that 2.7 million lives are lost to insufficient consumption of fruit and vegetables, and inadequate intake of these foods is considered a top risks factor for mortality worldwide [142]. Additionally, researchers show that a healthy diet high in fruits and vegetables can be considered a cost-effective treatment modality for disease prevention for populations who are considered "high-risk" for developing T2DM, MetS, and CVD [143-145]. Young adults and those attending universities may be considered a high-risk population because of their poor lifestyle choices that can continue into adulthood [88]. In 2003, the Behavioral Risk Factor Surveillance System reported that $78 \%$ of young adults (18-24) reported consuming less than 5 servings of fruits and vegetables per day [24]. Therefore, an intervention high in fruit and vegetables may decrease risk of disease for this younger population of college-aged individuals.

The human microbiome, sometimes referred to as the "forgotten organ" or "second genome", may have an even greater role than the human genome in the pathogenesis of obesity 
and inflammation than currently realized. Research has shown that the gut bacteria of a lean animal exhibits a different microbial profile than their obese counterparts independent diet [146] or genetics [73]. Microbiota of an obese animal is typically characterized by an increase in bacteria in the phylum Firmicutes and a decrease in Bacteroidetes compared to their lean counterparts. Moreover, germ-free mice colonized with microbiota from obese mice showed increased body fat and total energy content, suggesting that obese animals are able to extract more energy out of their diets [147]. Other researchers have found similar results of reduced Bacteroidetes and increased Firmicutes in obese humans compared to lean counterparts [148], although these studies are less well controlled than animal studies and have yielded varying results [149].

Based on a connection between nutrition and health, research is being directed on how nutrients modulate the human microbiome, and how the microbiome influences the host. It has been reported that dietary alterations are responsible for $57 \%$ of interpersonal variation of the gut microbiome, whereas genetic background explains $12 \%$ [150]. When dietary manipulation of the gut microbiome has occurred, common strategies have been through consumption of either probiotics or prebiotics [67], while other studies have examined the impact of comprehensive dietary manipulation on intestinal bacteria in individuals with MetS risk [55, 59, 74]. Moreover, understanding the relationship between the composition of the gut and its metabolic activity after a diet intervention involves pairing taxonomic characterization (16S rRNA gene assays) with metabolic profiling (i.e. metabolomics) [151]. This type of research has helped evaluate mechanisms underlying taxonomic and metabolic shifts in various environments and disease states $[152,153]$. Some metabolites that have been linked to cardiovascular morbidities include triacylglycerols with low carbon numbers and double bonds [154], membrane phospholipids 
[155], and serum amino acids, specifically branched-chain amino acids (BCAA) [156]. Pederson et al. (2016) identified certain species of bacteria that drove the association between biosynthesis of BCAA and insulin resistance [80]. Identifying these mechanisms help in creating targeted therapies to diminish incidence of metabolic disorders. To date, however, studies integrating taxonomic and metabolomics data are lacking and most have independently analyzed microbial communities and metabolite profiles.

Given the complexity of the gut microbiota and the challenge of linking dietary, metabolic, and microbial ecology factors that drive disease, additional nutrition intervention studies focused on population-based guidelines, are necessary in individuals with increased obesity, T2DM, and MetS risk. Recent data obtained from college students $(n=360)$ from the University of New Hampshire, Rutgers University, and West Virginia University (WVU) demonstrate that freshman in the Appalachian region have 4-fold greater metabolic risk compared to other state institutions of higher learning [157]. Furthermore, incidences of CVD, obesity and T2DM are highly prevalent in Appalachia [106, 107]. The objective of the FRUVEDomics Pilot Study (Clinical Trial Record 1409433435) was to investigate the influence of an eight week diet intervention on broad-scale targeted and non-targeted metabolomics, microbiome, and cardiovascular function in young adults "at-risk" of the MetS. We report in the present study the diet-induced changes in the microbiome after the intervention along with the relationships between the microbiome, dietary, and serum amino acids (AA). 


\section{Methods/Materials/Analysis}

\section{Diet}

A total of 36 subjects participated in an 8-week nutritional interventional study, which was adopted from the 2010 Dietary Guidelines for Americans [158]. Subjects were randomized into three groups according to the Choose MyPlate recommendations: a diet high in fruit and vegetables (FRUVED- 50\% fruit and vegetables); a FRUVED diet with low refined carbohydrate (FRUVED+LRC); or a FRUVED diet low in fat (FRUVED+LF). A description of each dietary arm is presented in Table 1.

\section{Study Design and Diet quantification}

Prior to starting the intervention, all individuals were educated on his/her diet during group sessions, and were given written instruction and culinary items (i.e. knives, cutting boards, measuring cups and spoons, and storage bags). During the intervention, subjects tracked their detailed daily food and supplement intake (i.e. probiotics, multivitamin, etc.) in written logs and photographed the foods that they consumed to help assess portion sizes. All items were reviewed during a weekly one-on-one counseling session with a Registered Dietitian Nutritionist (RDN). This intense intervention helped ensure compliance in the free-living design and personalization with the dietary recommendations. Following weekly counseling sessions and using each participants' 24 hour dietary recall, the RDN analyzed food components (\% macronutrients, fiber, sugar, empty calorie and fruit and vegetable consumption) using nutrition analysis software (Nutritionist Pro ${ }^{\mathrm{TM}}$, Axxya Systems LLC, Redmond, WA). These analyses were used to give personalized feedback for each participant (i.e. fruit and vegetable consumption, empty calorie adjustments, and fat intake). Additionally, physical activity was measured subjectively (activity 
logs) and objectively (accelerometers; ActiGraph) in order to assure that any measured changes were not due to alterations in physical activity. More details on the methodology and theory of the dietary intervention can be found in another manuscript (See Chapter II: Behavior Paper).

\section{Laboratory Measurements}

Body fat (BodPod), height (electronic Stadiometer), waist, hip and neck circumference (Gulick tape), blood pressure (Omron digital cuff), and weight (Tanita Scale) were measured before and after the 8 week intervention. Blood samples were collected before, every other week during, and at the end of the intervention, for a total of four samples per participant.

Methodology for the complete metabolic panel was previously described (reference See Addendum: Metabolome Paper).

A targeted profile of serum amino acids was measured by gas chromatography tandem mass spectrometry (GC-MS/MS). Samples were prepared by solid phase extraction and derivatization using the EZ:faast ${ }^{\mathrm{TM}}$ GC-MS Free (Physiological) Amino Acid Analysis Kit (KG0-7166; Phenomenex, Torrence, CA) with minor modifications as described by Elmore et al. [159]. This method has been previously validated by Badawy et al [160]. Partners at WVU utilized a Thermo Scientific (Waltham, MA) TRACE 1310 series gas chromatograph coupled to a TSQ8000 triple Quadrupole mass spectrometer for the analyses. Sample injections were handled by an autosampler (AI/AS1310 Thermo Fisher Scientific). Measurement of AAs were performed by selected reaction monitoring and the ions with the strongest signal chosen using autoSRM (Thermo Fisher Scientific). Variations in extraction efficiency and instrument electronics were normalized by norvaline internal standard. To calculate concentrations, a fivepoint standard curve of $26 \mathrm{AAs}(10 \mu \mathrm{M}$ to $200 \mu \mathrm{M})$ was constructed for each reported amino acid (average $\mathrm{R}^{2}=0.9963$; average intra-day precision \% $\mathrm{CV}=4.92$; average inter-day prevision 
$\% \mathrm{CV}=9.52$ ). Instrumentation and quantitation were processed by XCalibur (Thermo Fisher Scientific).

Stool Sample Collection and DNA extraction

Fecal samples were collected by each subject using Easy Sampler® stool kits (ALPCO, Salem, New Hampshire) then delivered to a lab within 2 hours, and stored at $-80 \mathrm{C}$ for later processing. A direct PCR approach was used to amplify bacterial DNA for sequencing as described [161]. Small fragments of fecal samples ( 200 mg) were suspended in RNA/DNA-free water and a $25 \mu \mathrm{l}$ aliquot from each sample was processed using the Extract-N-Amp Plant PCR kit (Sigma-Aldrich, Inc.) following the manufacturers' instructions.

The PCR primers for the V3 to V4 regions of the 16S ribosomal RNA gene were as described previously [162]. High-pressure liquid chromatography purified PCR primers for the V3 and V4 regions primers were obtained from Invitrogen (Grand Island, NY, USA). Bacterial DNA was amplified using an AccuPrime PCR Kit (Invitrogen Life Technologies, Grand Island, NY, USA) on a Techne Genius Model FGEN02TP Thermal Cycler using the following conditions: $95^{\circ} \mathrm{C}$ for 6 minutes denature; $95^{\circ} \mathrm{C}$ for 2 minutes, $50^{\circ} \mathrm{C}$ for 2 minutes, $72^{\circ} \mathrm{C}$ for 2 minutes 30 cycles; $72^{\circ} \mathrm{C}$ for 4 minutes extend. Each reaction contained $1 \mathrm{U}$ DNA polymerase, 5 $\mu$ 10x buffer 1(600 mM Tris-SO4 (pH 8.9), $180 \mathrm{mM}(\mathrm{NH} 4) 2 \mathrm{SO} 4,20 \mathrm{mM} \mathrm{MgSO} 4,2 \mathrm{mM}$ dGTP, 2 mM dATP, 2 mM dTTP, 2 mM dCTP, thermostable AccuPrime ${ }^{\mathrm{TM}}$ protein, 10\% glycerol), $20 \mu \mathrm{M}$ forward primer, $20 \mu \mathrm{M}$ reverse primer and DNA in a total volume of $50 \mu \mathrm{l}$. PCR reactions were performed in triplicate and reaction products were pooled prior to purification. PCR products were purified with Ampure XP beads (Beckman Coulter Life Sciences, Indianapolis, IN) per manufacturer's instructions. 


\section{S rRNA sequencing and processing}

Sequencing (2 x $150 \mathrm{bp}$ ) was performed using an Illumina MiSeq (San Diego, CA) in the Genomics Core Facility at WVU and then the paired ends were merged with FLASH (FLASH: fast length Magoc and Slazberg Bioinformatics 27:21 (2011[O1]). Quantitative Insights Into Microbial Ecology (QIIME v1.9.1 http://qiime.org) [163] was used to split the libraries and pick open-reference operational taxonomic units (OTUs) at $97 \%$ similarity. OTUs were first filtered by excluding those below a minimum threshold count of 500. Next, OTUs were hierarchically summed and normalized to relative abundance at all taxonomic levels. Features when then filtered if they met a cut-off of at least $17 \%$ of the sample having a minimum relative abundance of 0.005 , leaving a total number of features to 18 ( 5 phyla and 13 family taxa). Alpha-diversity measures including Chao-1, number of observed species, and phylogenetic distance were then determined.

\section{Statistical and Microbial Analysis}

Data are presented as means with standard deviations (SD) unless stated otherwise. Summary statistics were calculated for demographic characteristics. Outcome distributions for clinical and dietary data were examined and transformed if necessary, which included logtransformation, cubed root or square-root. Arcsine square root transformations were undertaken for relative abundance of the microbiome for regression analysis. A repeated measures ANOVA (RM-ANOVA) was used to assess effects of group, time, and the group by time interaction on AAs, which included 4 time points (bi-weekly).

Paired sample testing with inverse beta binomial distribution was used to assess changes in microbiome from baseline to post [164] and Bonferroni adjustment was utilized for multiple tests. To predict fecal community function, we used PICRUSt (phylogenetic investigation of 
communities by reconstruction of unobserved states-http://picrust.github.io/picrust/) based on data from the 16S rRNA data before and after the intervention [165]. The predicted Kyoto Encyclopedia of Genes and Genomes (KEGG) orthologs [166] were summarized to level 2 functional categories and significant differences in categories across time were identified by a two-sided Wilcoxon Signed Rank matched pairs test.

To find relationships among bacteria, diet, and serum AAs, an initial stepwise multiple linear regression was used to calculate relationships between each filtered taxa (phylum n=5; and family $n=13-$-refer to microbiome processing and filtering section) and groups of diet (19 each) variables and AAs (20 each). Then a separate stepwise multiple linear model was conducted joining the significant variables from each group for each individual taxa.

A p value $<0.05$ was considered statistically significant. Unless otherwise stated, Benjamini-Hochberg adjustment was utilized for multiple tests with a False Discovery Rate cutoff of $\mathrm{q}<0.25$. All analyses were conducted using SAS $\left(\right.$ SAS ${ }^{\circledR}$, Version 9.3, SAS Institute Inc., Cary, NC, Copyright @2002-2010), JMP (JMP®, Version Pro 11, SAS Institute Inc. Cary, NC, Copyright @2013) and R (R Core Team (2013), Vienna, Austria. URL http://www.Rproject.org/.).

\section{Results}

\section{Participant characteristics}

Characteristics of the participants at screening are provided in Table 2. Average age was $23.2 \pm 2.5$ years, and $42 \%$ men and $58 \%$ women started the intervention. There were 22 Caucasians, 4 African-Americans, 4 Asians, 4 Hispanics, and 2 individuals that identified as 'Other'. Body mass index (BMI) at baselines was $27.2 \pm 6.0$ and $53 \%$ of the participants grew up 
in the Appalachian region. Eighty-six percent of participants were considered "at-risk" of developing MetS, which was a score of $\geq 3$ points on the tool developed by the research investigators of this study (See Addendum: Risk Score Abstract). The only significant differences among participants in the three dietary arms at baseline were the percentage of Caucasian individuals in the FRUVED group, and younger age of FRUVED+LRC group. Following the 8 week intervention, 6 individuals were eliminated from analyses due to noncompliance with the combination of dietary intake, physical activity recommendations, or use of regulated medications such as antibiotics. There were no demographic differences between those starting the intervention $(n=36)$ and the individuals who were included in the microbiome and metabolomic analyses $(n=30)$.

\section{Nutrient Intake}

The mean daily intake for the 30 compliant participants of various dietary variables at baseline and after the 8 week intervention were reported and recorded elsewhere (See Addendum: Metabolome Paper). The diets were designed so that daily energy remained consistent throughout the 8 weeks, with changes coming only from the different food groups. There were no significant difference in total calories or in the percentage coming from protein across time or by group. Over the 8 weeks, the participants significantly increased grams of fiber, grams of total sugar, and total cups of fruits and vegetables. Both insoluble and soluble fiber increased with the intervention and was the only significant differences seen between groups. Additionally, there were significant decreases over time in the percentage of calories from fat, saturated fat, and empty calories (i.e. calories that provide extra sugar and fat). Other than the breakdown of fiber, there were no significant differences in any dietary variables by groups, 
indicating all participants followed the same type of dietary intervention throughout the course of the intervention.

\section{Anthropometrics, Biochemical and Amino Acid Metabolites}

Following the intervention, researchers reported significant differences in various anthropometric and biochemical measures (See Addendum: Metabolome Paper). Significant decreases were observed with waist circumference, neck circumference, systolic blood pressure, and high density lipoprotein (HDL) at the end of the intervention. The intervention also resulted in significant increases in potassium levels. No significant main effects between groups or the interaction of group or time were observed. We also observed changes in some AAs after the intervention; Glutamic acid (Glu), Tryptophan (Trp), and Cysteine (Cys) increased as intervention progressed (time effect $p<0.05$ ), while levels of Ornithine (Orn) were lowered with time $(P<0.05)($ Table 5).

\section{Fecal Microbiota Composition and Changes}

The distributions of the bacteria using the fecal samples obtained pre and post intervention were analyzed at the phyla and family level (Figure 1). The microbiota in all participants was dominated by the two bacterial phyla Bacteroidetes and Firmicutes, with lower levels of Proteobacteria and Verrucomicrobia. Phylum Bacteroidetes was represented by 5 major families, which included Bacteroidaceae, Porphyromonadaceae, Rikenellaceae, Prevotellaceae, and Barnesiellaceae. Major families of Firmicutes were Ruminococcaceae, Lachnospiraceae, Clostridiales Cluster, and Veillonellaceae. Lastly, Proteobacteria and Verrucomicrobia had one dominant family each, which were Alcaligenaceae and Verrucomicrobiaceae respectively. 
Paired t testing using a binomial distribution showed significant decreases following the intervention in the phyla Firmicutes (fold change $=-1.3$, adj $\mathrm{p}=0.23$ ), while Bacteroidetes significantly increased following the intervention (fold change=-1.3, adj $\mathrm{p}=0.23$ ) (Figure 2). We also observed significant decreases in Cyanobacteria (fold change $=-1.9$, adj $p=0.005$ ). At the family level, decreases were observed in a Clostridiales Cluster (fold change=-1.7, adj p=0.003), Ruminococcaceae (fold change $=-1.3$, adj $\mathrm{p}=0.22$ ), Barnesiellaceae (fold change $=-4.0$, adj $\mathrm{p}<0.001$ ), and Paraprevotellaceae (fold change $=-4.0$, adj $\mathrm{p}<0.0001$ ). A significant increase was seen in S-24 7 (fold change $=4.0$, adj $\mathrm{p}<0.0001$ ), Alcaligenaceae (fold change $=1.8$, adj $\mathrm{p}=0.0006$ ), and Bacteroidaceae (fold change $=1.3$, adj $\mathrm{p}<0.05$ ) post intervention across all diets.

\section{Microbial Metagenomics}

PICRUSt was used to infer putative metagenomes from the 16S rRNA gene profiles in order to investigate microbiome functions before and after a healthy diet intervention. The representation of mean different proportions are listed in Figure 3 for the identified categorical $(n=39)$ functions. Comparing the functional categories of bacterial genes in the microbiomes of our 30 compliant individuals before and after the intervention, there was no significant differences. Together these results suggest that an 8 week nutrition intervention did not alter the microbiome metabolic functions.

\section{Microbiome Interactions with Diet and Amino Acids}

Linear regressions between microbiota and diet variables were conducted at baseline and post intervention separately. At baseline, Proteobacteria was positively related to cups of fruits and vegetables (estimate $=0.03, p=0.01$, Figure $4 a$ ), polyunsaturated fatty acid and monounsaturated fatty acid intake (estimate $=0.08, \mathrm{p}=0.006$ and estimate $=0.04, \mathrm{p}=0.02$ respectively). Cyanobacteria was negatively related to total sugar (estimate $=-0.001, p=0.03$, 
Figure $4 \mathrm{~b}$ ) and positively related with empty calories (estimate $=0.02, \mathrm{p}=0.05$ ). No other relationships were found with other major phyla such as Firmicutes and Bacteroidetes (Table 4). At the family level, Alcaligenaceae was negatively related to threonine (estimate $=-0.001, \mathrm{p}=$ 0.03, Figure 4c). Porphyromonadaceae was negatively related to total sugar consumption (estimate $=-0.001, p=0.04$ ) and cups of fruit and vegetables (estimate $=-0.07, p=0.04$, Figure 4d) and positively related to saturated fatty acid intake (estimate $=0.06, \mathrm{p}=0.04)$ (Table 4).

Following the intervention, Proteobacteria was positively related with glutamic acid (estimate $=0.003, p=0.02$, Figure 5a). Verrucomicrobia was negatively related to alanine (estimate $=-0.0005, p=0.03$, Figure 5b), and aminobutyric acid (estimate $=-0.05, p=0.02)$ and positively related to asparagine (estimate $=0.002, \mathrm{p}=0.02)($ Table 5). Significant relationships were observed at the family level after the intervention (Table 5), where Alcaligenaceae was positively related to insoluble fiber (estimate $=0.05, \mathrm{p}=0.02$, Figure $5 \mathrm{c}$ ), percentage of calories from protein (estimate $=0.09, p=0.01)$, and serine (estimate $=0.07, p=0.01)$.

Verrucomicrobiaceae was negatively related to alanine (estimate $=-0.0004, \mathrm{p}=0.03$ ), and aminobutyric acid (estimate $=-0.05, \mathrm{p}=0.02)$, and positively related to asparagine $($ estimate $=$ $0.002, \mathrm{p}=0.02$, Figure 5d).

\section{Discussion}

An 8 week dietary intervention based on the 2010 Dietary Guidelines for Americans significantly increased consumption of fiber, fruit and vegetables, with concomitant decreases in saturated fat and empty calorie consumption. Apart from fiber breakdown, no other dietary factors differed among the three subgroups of the FRUVEDomics study; therefore the changes in the microbiota and other clinical measurements were driven by all individuals shifting from a Western diet (high in simple sugars and fats) to a healthier alternative (high in fiber, fruits and 
vegetables, and low in fat). No changes were found in other health behaviors from baseline to post intervention (See Chapter II: Behavior Paper).The intervention also increased antiinflammatory markers and decreased fatty acid metabolites associated with inflammation (See Addendum: Metabolome Paper), altogether making these observed changes clinically important among a population with an elevated number of risk factors for disease, but otherwise healthy.

The present study investigated the impact of a healthy diet on gut microbial composition in the young adult population who attend a university located in the Appalachian region where there is high magnitude of disease [106-109]. We sought to answer the underlying question of whether an intervention with high levels of dietary fiber can modulate the gut microbiota of humans following a Western-style diet with low fiber at baseline. Obese individuals have been reported to have a decrease in Bacteroidetes and an increase in Firmicutes $[56,167]$. We found increases in Bacteroidetes and decrease in Firmicutes following the intervention, which resembled that of class "lean" phenotypes or when weight loss in humans occurred by either a carbohydrate or fat-restricted diet [73]. Although total body weight was approaching significant decreases over time $(\mathrm{p}=0.07)$, we observed decreases in waist circumference where increase in this anthropometric measurements has been associated with cardiometabolic disease risk [168]. However, it is important to note that others have observed different results at the phylum level when it comes to disease pathogenesis. In a recent review, investigators reported higher Bacteroidetes and lower Firmicutes in type 2 diabetics and obese subjects [149]. This effect could be due to proinflammatory efforts of lipopolysaccharide (LPS), which is an essential component of cell walls of gram-negative bacteria in the phylum Bacteroidetes. In addition to these results, we observed a significant decreases in the phylum of Cyanobacteria in our subjects that contributes to a small proportion of total gut bacteria. To our knowledge, no researchers 
have reported this change in human studies following a dietary intervention. Therefore we speculate that our finding was due to a low amount of people who had this bacteria in their gut prior to the intervention or caused by environmental contaminants when samples were processed. Altogether, this novel finding along with other conflicting results presents the need for more research using similar strategies, study populations, and dietary methods.

Recently, dietary approaches to counteract obesity and metabolic disease by modifying microbiome have focused on increasing fiber consumption [169]. In the present study, our participants increased carbohydrate intake from 43 to $54 \%$ of total calories and averaged $34 \mathrm{~g}$ of dietary fiber (range 15-76g) after 8 weeks. This indigestible polysaccharide is fermented in the distal colon via bacterial enzymes [61]. The end production is short chain fatty acids (SCFAs) (i.e. acetate, propionate and butyrate), which lead to diverse and beneficial effects to the host [170] such as reduced inflammation in various tissues [171] and promotion of satiety [172]. Although we did not measures SCFAs, it has been found that those who consumed higher intake of dietary fiber and whole plant foods tend to have higher concentrations of SCFAs in their feces [170], conferring a protective role from irritable bowel syndrome and other inflammatory disease $[65]$.

Influential studies in genetically obese mice found that SCFAs acts as ligands for Gpr41, a $\mathrm{G}$ protein-coupled receptor expressed by a subset of enteroendocrine cells in the gut epithelium, which contributes to host energy harvest when coupled with certain microbiota. Researchers showed that when germ-free Gpr41a -/- mice were colonized with Bacteroides thetaiotaomicron and the archaeon Methanobrevibacter smithii, they displayed leaner phenotypes compared to conventionally raised wild-type littermates, despite similar chow consumptions [66]. The researchers associated their findings with increased expression of 
peptide YY (PYY), a gut hormone that reduces food intake [173]. Our study showed significant increases in Bacteroidaceae, the higher taxonomic unit of B. thetaiotaomicron, after the 8 week intervention. Similarly, Bacteroidaceae increased in mice after consumption of guar gum or inulin [174]. The authors reported that the increase in Bacteroidaceae, as well as Porphyromonadaceae and Verrucomicrobiaceae, were due to expression of glycosidases that are present in this group of bacteria. Therefore, the increases in Bacteroidaceae, and to a lesser extent, Verrucomicrobiaceae, that we saw among our participants could be due to the increased consumption of fiber, leading to the increased function of these bacteria. Overall, the presence of these bacteria in the gut may augment satiety and limit weight gain.

In addition to increases in Bacteroidaceae, we saw a significant increase in the S24-7 family, and although not significant, an increase in Prevotellaceae. Recently, bacteria in the family S24-7, the order Bacteroidales, and genus Prevotella (all Bacteroidetes) have been shown to act in favor of type I diabetes protection because of their strong correlation to systematic FoxP3+ regulatory $\mathrm{T}$ (Treg) cells in non-diabetic mice [175]. On the other hand, members of the family Lachnospiraceae, and bacteria of the genus Ruminococcus, and Oscillospira were shown to be in favor of pathogenesis (all Firmicutes) [175]. Similarly, we saw a significant decreases in the families of Ruminococcaceae (higher taxonomic rank of Ruminococcus and Oscillospira) and although not significant, a decrease in Lachnospiraceae. Furthermore, Akkermansia muciniphila, belonging to the phylum Verrucomicrobia, has been directly implicated in improving MetS and CVD [69]. When A. muciniphila was given to an obese animal, researchers saw improved glucose tolerance [68]. This species has also shown to be reduced among obese subjects, but increases after gastric bypass $[150,176]$. Our findings illustrate increases among Verrucomicrobiaceae, the family of A. muciniphila, yet this was not significant. 
Previous studies have shown that individuals with MetS have reduced abundance of Bacteroides [75], which has important saccharolytic activity [177] and can improve gut barrier by inducing goblet cell proliferation and mucus production [178]. After 8 weeks of a high fruit and vegetable diet, our participants increased Bacteroidaceae, the family that contains the commensal genera Bacteroides. In a similar study, researchers investigated the effect of five experimental diets in subjects who were "at-risk" of MetS $(n=88)$ and consumed one of five randomized diets for 4 weeks [74]. The five diets consisted of the following: high monounsaturated fat/high glycemic index; high monounsaturated fat/low glycemic index; high carbohydrate/high glycemic index; high carbohydrate/low glycemic index; and high saturated fat. Results after 24 weeks showed that the groups who increased carbohydrate and reduced dietary fat increased numbers of Bacteroides, which were inversely associated with body weight $(r=-0.64)[74]$. Haro et al. reported restorations of Bacteroides and other potentially beneficial members of bacteria after patients with MetS consumed a Mediterranean diet, high in carbohydrates and low in fat, for 2 years [75]. Altogether, our results strength the hypothesis that modification of the gut microbiota can occur after consumption of a healthy diet based around the national guidelines and improve metabolic dysfunction through various mechanisms.

Besides changes in the microbiota, we observed relationships among certain microbiota with dietary factors and amino acid metabolites, providing functional information associated with a high-fiber, low-fat diet. Our intervention induced changes in levels of certain AAs (i.e. Glu, Trp, Cys, and Orn), even though there was no significant change in total protein intake over the 8 weeks. However, it is documented that AA can be degraded in the intestine by the microbiota [179] and could be the reason why we observed relationships with the microbiome. Trp in particular can be converted into indoleproprionate by tryptophanase produced by 
Clostridia bacteria [180] and has been shown to have positive correlations with changes in gut bacteria from the Lachnospiraceae family [181]. Although we did not observe those specific relationships, others have shown associations between a diet high in protein and fat and increased Proteobacteria and Bacteroidetes [57]. We observed a relationship between Proteobacteria and Glu, an AA involved in protein synthesis. Additionally, Alcaligenaceae, a lower taxonomic unit of Proteobacteria, was found to have a positive relationship with the percent of total calories from protein. Furthermore, we saw other relationships between families of bacteria and AAs. Despite these indications and novel findings, more work is needed in this area, especially between protein fermentation and gut bacteria in human studies.

There are a few limitations of the current study. Our small sample size limited the power in stratifying by demographics or dietary arms in the statistical tests. Additionally, a longer consumption period may have produced different alterations in the gut microbiota, however other studies have utilized short dietary interventions and have elicited gut microbiota shifts [55, 182]. We found no significant changes in microbial metagenomes after the intervention, which could also be attributable to the length of our intervention. Lastly, some of the conclusions that were drawn with other literature on the mechanisms of bacteria should be taken lightly as we studied higher taxonomic levels to reduce false associations at the genus levels because of our small sample size.

\section{Conclusion}

In conclusion, this study illustrates that a short-term, dietary intervention, using the 2010 Dietary Guidelines for Americans, impacts gut microbial composition in a young adult population who is "at-risk" of disease and habitually follow a low-fiber Western diet. We report increases in Bacteroidetes and decreases in Firmicutes, which was accompanied by a decrease in 
waist circumference among our study participants. In addition we observed increases in families of bacteria that have been linked to lowered inflammation, increased SCFA production, and improved gut epithelial integrity. Lastly, the correlation analysis results suggest that the fecal microbiome-dietary/amino acid relationships might inspire potential methods for preventing and reducing disease by personalized nutrition methods. The current study supports evidence that population-based diets are effective in modulating the gut microbiota and potentially influence metabolic disease. Future research would benefit using the same dietary intervention, with longer consumption time, in a diseased cohort to examine the relationship between microbial changes and various metabolic end products in the host 
Tables/Figures

Table 1: Dietary Arms in FRUVEDomics Study

\begin{tabular}{|ll|}
\hline Diet & Main Components \\
FRUVED & $\begin{array}{l}50 \% \text { of plate fruit and vegetables; equivalent to } 2.5 \text { cups vegetables for women and } 3 \text { cups for men daily. } \\
\text { Fruit intake was } 2 \text { cups daily for all individuals. }\end{array}$ \\
FRUVED+LRC & $\begin{array}{l}\text { FRUVED diet plus choose low-glycemic foods (particularly non-starchy vegetables, legumes, and temperate } \\
\text { fruits) and limit intake of high-glycemic foods (i.e. refined grains, starchy vegetables, fruit juices, and } \\
\text { sweets). }\end{array}$ \\
FRUVED+LF & $\begin{array}{l}\text { FRUVD diet plus 30\% of their calories from fat, with only 10\% or less coming from saturated fat, and 300 } \\
\text { mg of cholesterol per day. Participants were told to make most of their protein and dairy sources lean. }\end{array}$
\end{tabular}

FRUVED (High Fruit and Vegetables) diet was set by national standards from the Center for Disease Control and Prevention based on the MyPlate recommendations [7]. The FRUVED+LRC (High Fruit and Vegetables, low refined carbohydrate) diet has standards published elsewhere [21] and the FRUVED+LF (High fruit and vegetables, low fat) diet was based off the American Heart Association guidelines [6]. 
Table 2: Demographic and Health Related Characteristics at Baseline Evaluation

\begin{tabular}{|c|c|c|c|c|c|}
\hline & $\begin{array}{l}\text { Total } \\
(\mathrm{n}=36)\end{array}$ & $\begin{array}{c}\text { Included }^{\mathrm{a}} \\
(\mathrm{n}=30)\end{array}$ & $\begin{array}{c}\text { FRUVED } \\
(\mathrm{n}=12)\end{array}$ & $\begin{array}{c}\text { FRUVED+LRC } \\
(\mathrm{n}=12)\end{array}$ & $\begin{array}{c}\text { FRUVED+LF } \\
(\mathrm{n}=12)\end{array}$ \\
\hline Age $(x \pm S D)$ & $23.2 \pm 2.5$ & $23.6 \pm 2.4$ & $23.7 \pm 1.5$ & $21.5 \pm 2.9^{* *}$ & $24.3 \pm 2.1$ \\
\hline Sex (\% male) & 42 & 47 & 42 & 42 & 42 \\
\hline \multicolumn{6}{|l|}{ Race/ethnicity (\%) } \\
\hline White & 61 & 67 & $84 * *$ & 41 & 58.3 \\
\hline African-American & 11 & 7 & $0 * *$ & 17 & 16.7 \\
\hline Asian & 11 & 10 & $0 * *$ & 17 & 8.3 \\
\hline Hispanic & 11 & 10 & 8 & $25 * *$ & 8.3 \\
\hline Other & 6 & 6 & 8 & 0 & 8.3 \\
\hline Body Mass Index $(x \pm S D)$ & $27.0 \pm 6.4$ & $26.0 \pm 4.2$ & $27.4 \pm 5.0$ & $26.5 \pm 8.1$ & $27.2 \pm 6.3$ \\
\hline From Appalachia (\%) & 53 & 60 & $75^{* *}$ & 42 & 41.7 \\
\hline \multicolumn{6}{|l|}{ MetS Risk Category (\%) } \\
\hline Low Risk & 14 & 17 & 25 & 8 & 8 \\
\hline Medium Risk ( $\geq 3$ points) & 58 & 60 & 42 & 59 & 75 \\
\hline High Risk ( $\geq 6$ points) & 28 & 23 & 33 & 33 & 17 \\
\hline
\end{tabular}

${ }^{\mathrm{a}}$ Individuals included in analysis based on high compliance score from RDN and no extreme changes in physical activity, stress and sleep over the intervention.

$* \mathrm{P}<0.05$ between total and included participants

$* * \mathrm{P}<0.05$ among intervention groups 
Table 3: Treatment effects on amino acids from pre to post intervention on compliant individuals $(\mathrm{N}=30)$

\begin{tabular}{lccccc}
\hline $\begin{array}{l}\text { Amino Acid } \\
(\mu \mathrm{M})\end{array}$ & $\begin{array}{c}\text { Pre Intervention } \\
(\mathrm{Mean} \pm \mathrm{SD})\end{array}$ & $\begin{array}{c}\text { Post Intervention } \\
(\mathrm{Mean} \pm \mathrm{SD})\end{array}$ & $\begin{array}{c}\text { Group Effect } \\
(\mathrm{p} \text {-value })\end{array}$ & $\begin{array}{c}\text { Time Effect } \\
(\mathrm{p} \text {-value })\end{array}$ & $\begin{array}{c}\text { Group*Time Effect } \\
(\mathrm{p} \text {-value })\end{array}$ \\
\hline Alanine & $351.0 \pm 73.8$ & $356.3 \pm 70.5$ & 0.63 & 0.90 & 0.22 \\
Glycine & $215.1 \pm 41.8$ & $216.8 \pm 45.9$ & 0.99 & 0.96 & $0.04^{*}$ \\
$\alpha-$ Aminobutyric acid & $19.8 \pm 10.6$ & $18.9 \pm 10.3$ & 0.53 & 0.87 & 0.21 \\
Valine & $204.7 \pm 43.1$ & $215.7 \pm 33.5$ & 0.56 & 0.08 & 0.62 \\
Leucine & $118.2 \pm 25.8$ & $120.2 \pm 21.2$ & 0.24 & 0.54 & 0.69 \\
Isoleucine & $66.6 \pm 15.4$ & $70.4 \pm 14.5$ & 0.27 & 0.16 & 0.38 \\
Threonine & $136.8 \pm 29.7$ & $142.4 \pm 24.1$ & 0.79 & 0.50 & 0.08 \\
Serine & $115.6 \pm 25.5$ & $113.7 \pm 22.8$ & 0.77 & 0.68 & 0.67 \\
Proline & $176.2 \pm 56.1$ & $171.2 \pm 52.0$ & 0.90 & 0.63 & 0.54 \\
Asparagine & $57.2 \pm 10.4$ & $53.3 \pm 11.9$ & 0.88 & 0.07 & 0.20 \\
Methionine & $20.9 \pm 5.4$ & $20.1 \pm 4.6$ & 0.38 & 0.44 & 0.20 \\
Glutamic acid & $25.0 \pm 10.1$ & $32.8 \pm 14.2$ & 0.25 & $<0.001^{*}$ & 0.20 \\
Phenylalanine & $60.5 \pm 10.1$ & $60.7 \pm 10.3$ & 0.83 & 0.83 & 0.84 \\
Glutamine & $545.0 \pm 121.6$ & $591.4 \pm 124.7$ & 0.91 & 0.06 & 0.61 \\
Ornithine & $63.2 \pm 25.5$ & $51.5 \pm 16.8$ & 0.48 & $0.001^{*}$ & $0.02^{*}$ \\
Lysine & $193.6 \pm 55.1$ & $198.6 \pm 51.7$ & 0.60 & 0.79 & 0.24 \\
Histidine & $86.5 \pm 10.2$ & $99.7 \pm 52.4$ & 0.54 & 0.16 & $0.04^{*}$ \\
Tyrosine & $56.5 \pm 15.5$ & $58.6 \pm 16.4$ & 0.99 & 0.49 & 0.40 \\
Tryptophan & $57.4 \pm 10.4$ & $64.8 \pm 12.0$ & 0.74 & $0.002^{*}$ & 0.64 \\
Cysteine & $34.0 \pm 4.4$ & $37.0 \pm 5.3$ & 0.59 & $0.001^{*}$ & 0.38 \\
\hline Amino & & &
\end{tabular}

Amino acid metabolites were collected from all individuals at baseline and post intervention. Repeated measures ANOVA was used to analyze the main effects of group (FRUVED, FRUVED+LRC, FRUVED+LF), time, and their interaction. Significant effects

ANOVA were followed by multiple comparisons using Tukey adjustment. Untransformed baseline and post intervention means and standard deviations are listed, along with $\mathrm{p}$ values for the main effects and interaction.

${ }^{a}$ Individuals included in analysis based on high compliance score from RDN and no extreme changes in physical activity, stress and sleep over the intervention.

$* \mathrm{P}<0.05$ 
Table 4: Multiple regression on phyla with dietary and serum amino acids at baseline $(\mathrm{N}=30)$

\begin{tabular}{|c|c|c|c|}
\hline Taxon & Effect & Slope & P-value \\
\hline \multicolumn{4}{|l|}{ Phylum } \\
\hline \multirow{3}{*}{ Proteobacteria } & Fruit and Vegetables (cups) & 0.03 & 0.01 \\
\hline & Polyunsaturated Fat & 0.08 & 0.006 \\
\hline & Monounsaturated Fat & 0.04 & 0.02 \\
\hline \multirow{2}{*}{ Cyanobacteria } & Total Sugar & -0.001 & 0.03 \\
\hline & Empty Calories & 0.02 & 0.05 \\
\hline \multicolumn{4}{|l|}{ Family } \\
\hline \multirow[t]{2}{*}{ Alcaligenaceae } & Threonine & -0.001 & 0.03 \\
\hline & Total Sugar & -0.001 & 0.04 \\
\hline \multirow[t]{2}{*}{ Porphyromonadaceae } & Fruit and Vegetables (cups) & -0.07 & 0.04 \\
\hline & Saturated Fat & 0.06 & 0.04 \\
\hline \multicolumn{4}{|c|}{$\begin{array}{l}\text { Amino acids ( } 20 \text { each) and diet (19 each) were collected at baseline along with the microbiome (18 OTUs each) for all } 30 \text { compliant } \\
\text { individuals. First, a stepwise linear regression was conducted on separate OTU for each individual class of variables at baseline. A } \\
\text { secondary stepwise linear regression was conducted on each OTU combining all significant variables from each set of classes on the } \\
\text { first regression for baseline. At each step of the regression, Benjamini-Hochberg adjustment was conducted using a FDR of } 0.25 \text {. The } \\
\text { table lists the parameter estimates and the adjusted p-values for the final model of significant variables. }\end{array}$} \\
\hline
\end{tabular}


Table 5: Multiple regression on phyla with dietary variables and serum amino acids at post intervention $(\mathrm{N}=30)$

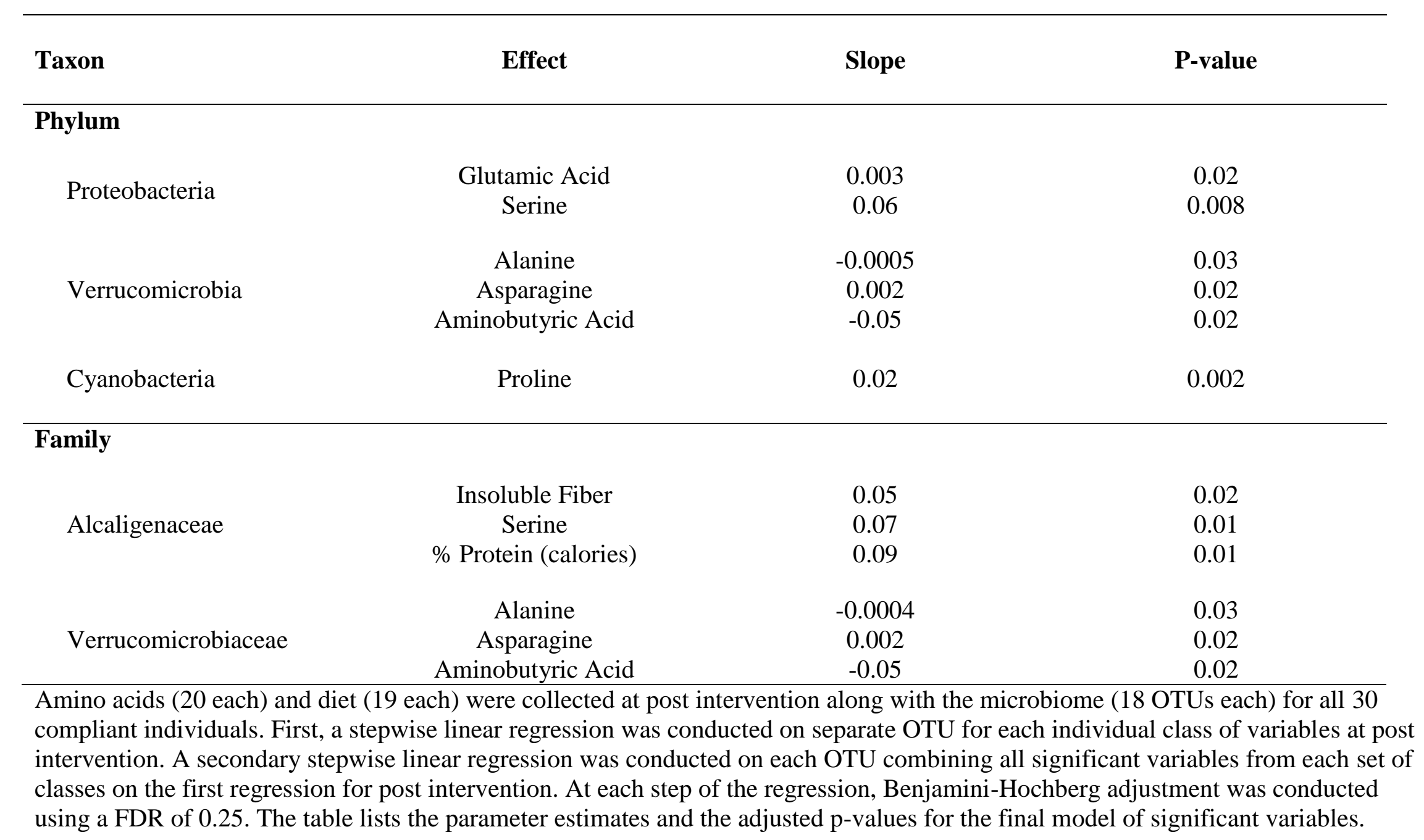


$\underline{\text { Pre Intervention }}$

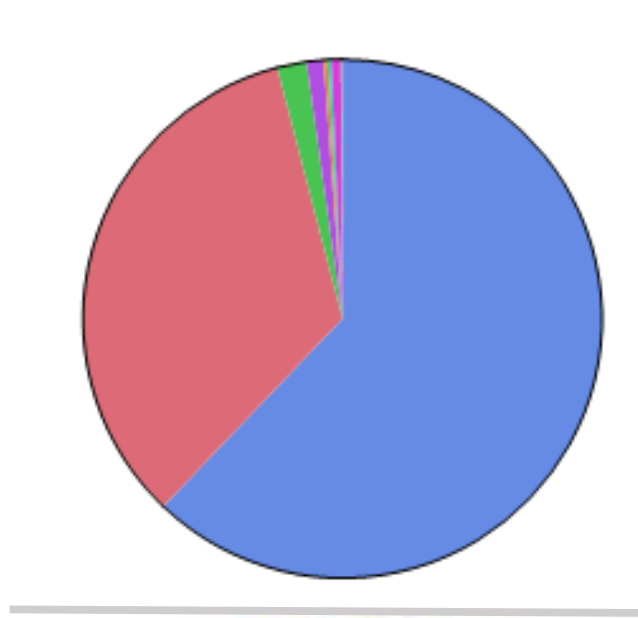

Post Intervention

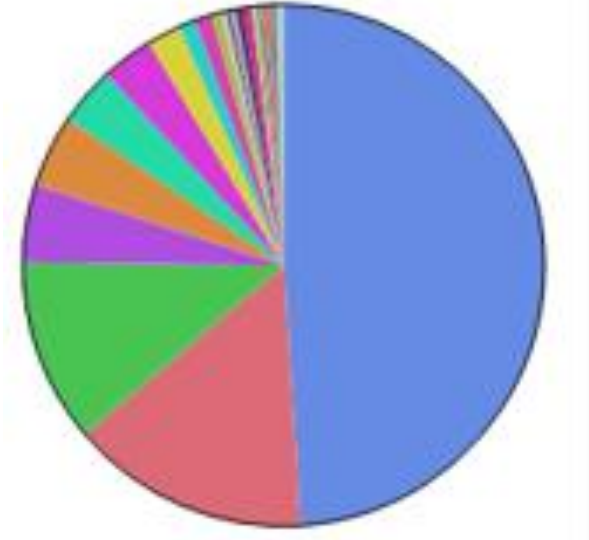

Figure 1: Microbiota composition of the study population after Baseline and Post Intervention for Phylum and Family. Fecal samples were collected for every individual at baseline and post intervention. Average relative abundance across all samples at pre and post intervention are presented. Top of chart represents phyla and bottom represents family after filtering OTUs at a minimum threshold of 500 .
Phylum

Bacteroidetes
Firmicutes
Proteobacteria
Verrucomicrobia
Actinobacteria
Tenericutes
Cyanobacteria
Other
Elusimicrobia
TM7
Lentisphaerae

Family

Bacteroidaceae Ruminococcaceae - Tachnospiraceae

Clostridiales_f

Porphyromonadacese Rikenellaceae

Prevotellacese Veillonellaceae Verrucomicrobiacese Alcaligenaceae Anaeroplasmataceae Bacteroidales f Bamesiellacese Bifidobacteriaceae Campylobscteracese Camobscteriacese Christensenellaceae Clostridiacese Clostridisles Other Coriobacteriaceae
Desulfovibrionaceae Elusimicrobiacease Entercbacteriacese

Erysipelotrichacease familyother ML615J-28f Mogibacteriaceae Moraxellaceae Neisseriacese

Odoribacteraceae

Paraprevotellaceae Pasteurellaceae

Peptostreptococcaceae Pseudomenadaceae RF32_f

RF $39_{-} f$

S24-7

SHA-98_f

Streptococcacese

Streptophyta_f 
Verrucomicrobiaceae

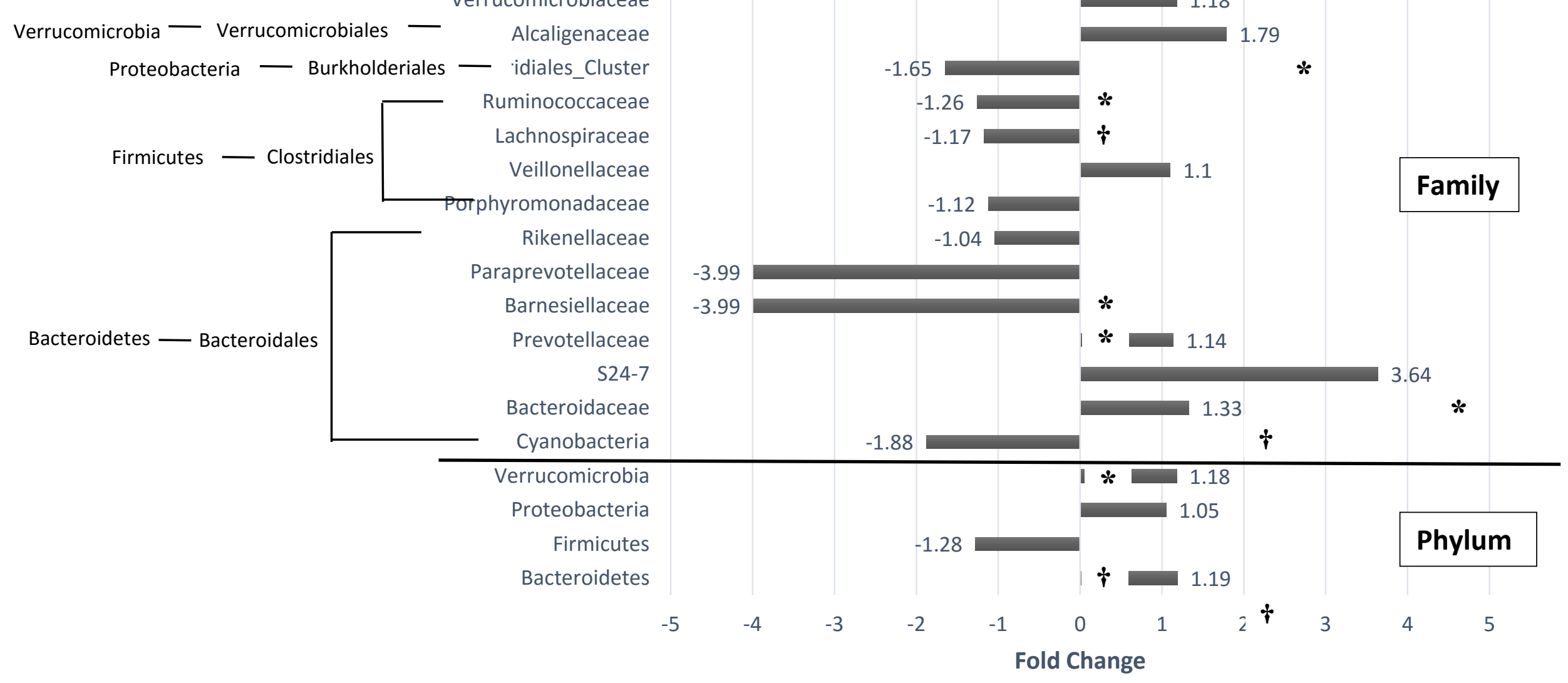

Figure 2: Microbiome changes in phylum and family taxa using inverted beta binomial paired testing. Values represent common fold change by IBB paired testing, calculated by dividing the bacterial relative abundance at 8 weeks by baseline. *, P $<0.05$ significant differences from baseline. $\dagger, \mathrm{P}<0.25$ in the post hoc Benjamini Hochberg multiple comparison tests between the bacterial abundance of filtered OTUs from baseline. 


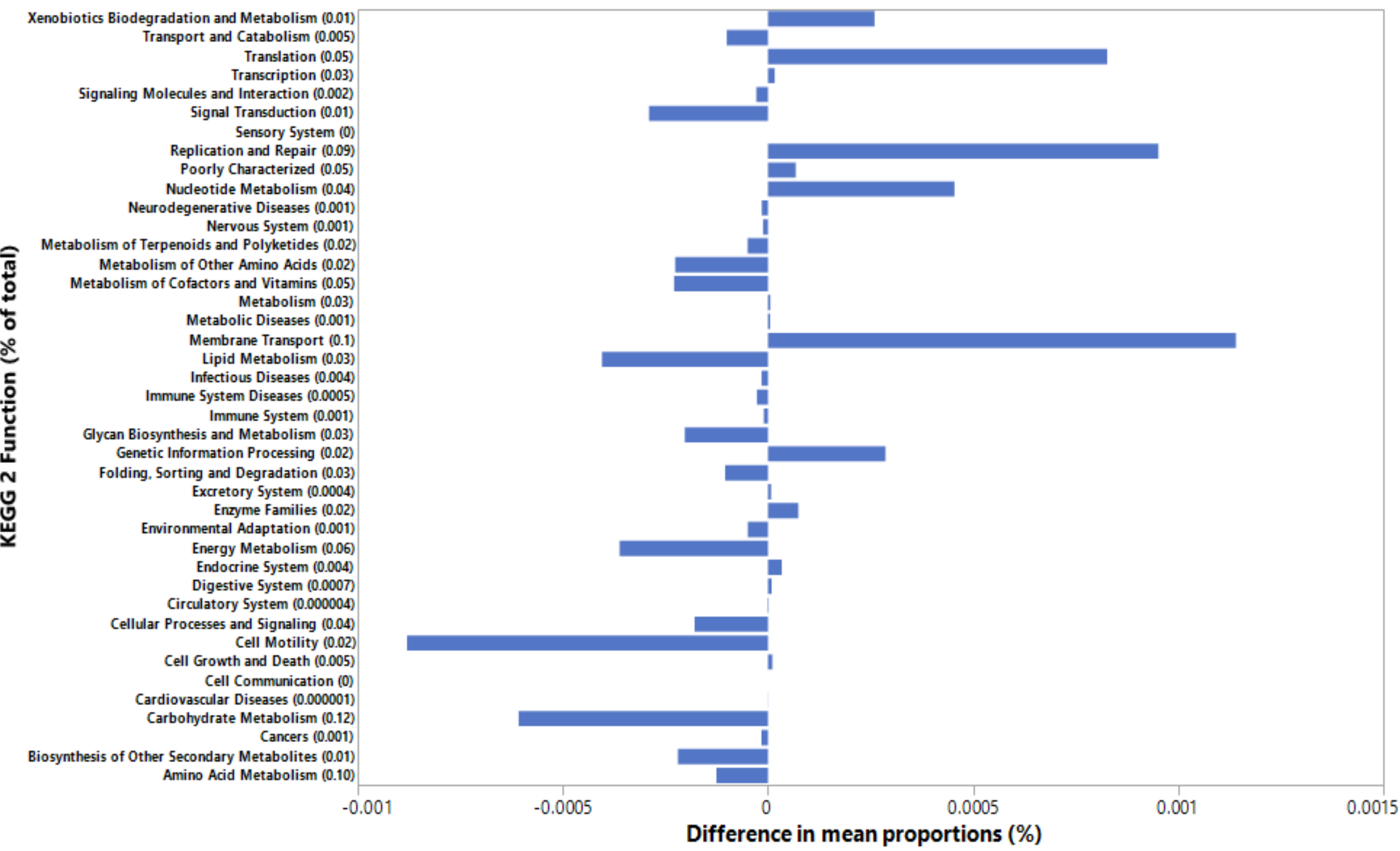

Figure 3: Bacterial metagenomes predicted from microbial community identities. Gene function categories were from level 2 of the KEGG pathways. Difference in mean proportions was based on total functions. A two-sided Wilcoxon Signed Rank matched-pairs test with Benjamini Hochberg adjustments $(\mathrm{q}<0.25)$ was used to determine significant difference in mean proportions from baseline to post intervention. ${ }^{*} \mathrm{P}<0.05$ 


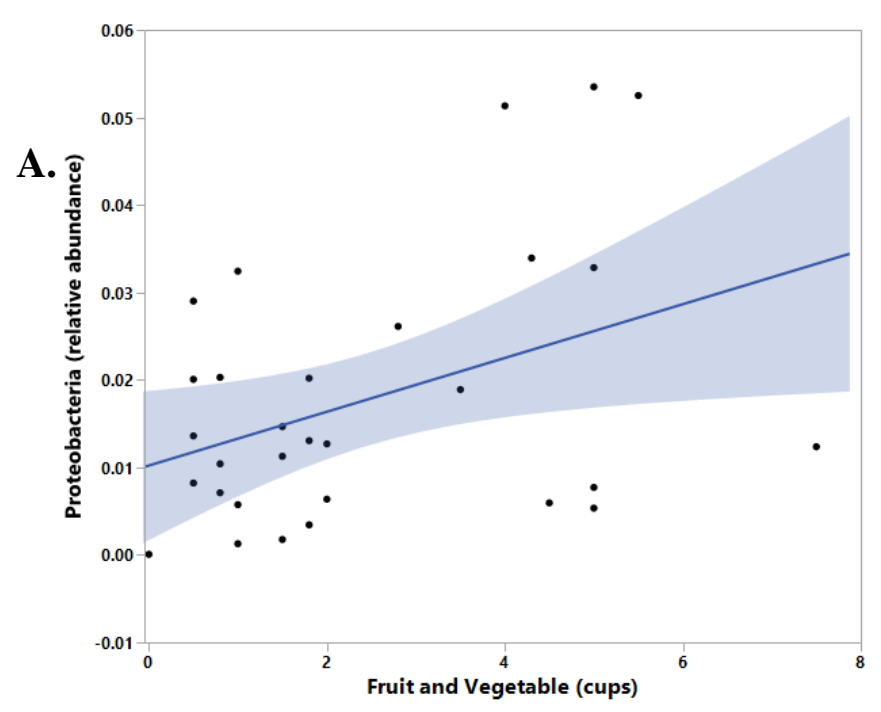

\section{Phylum}

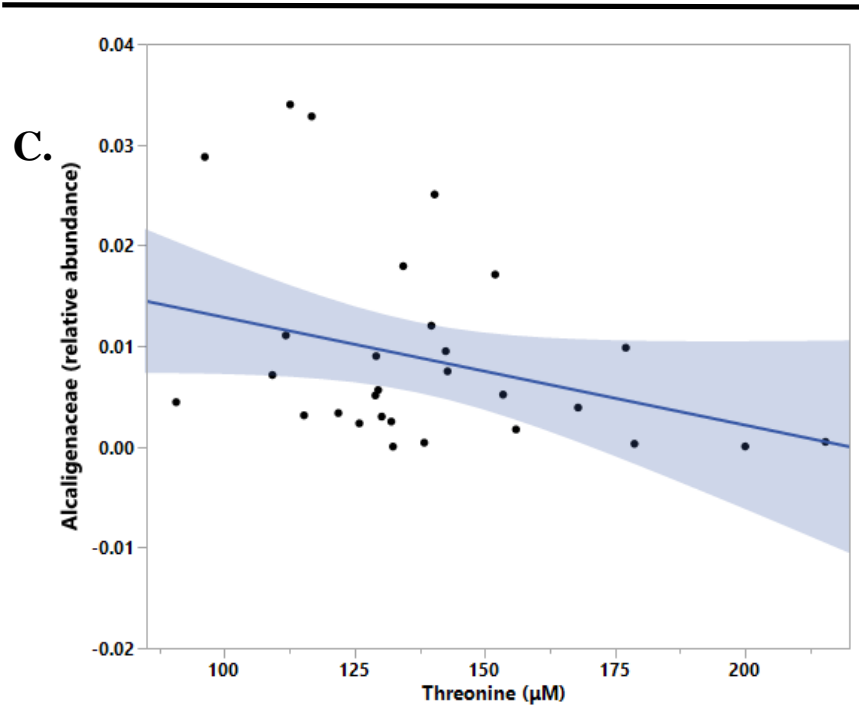

\section{Family}
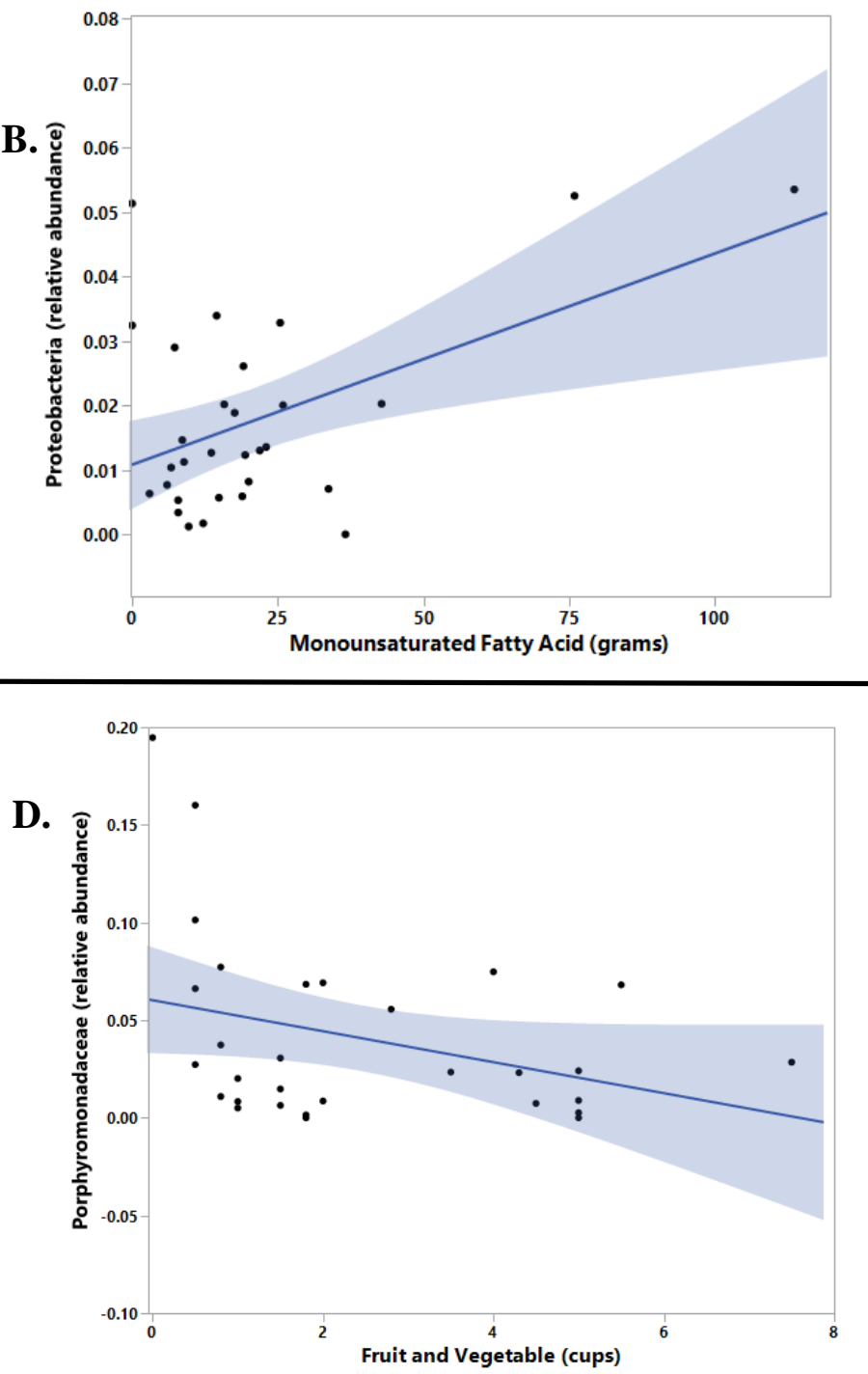

Figure 4: Baseline significant stepwise regressions $(b=$ slope $)$ among taxon relative abundance, dietary factors, amino acids. (a) Proteobacteria and Fruit and Vegetables $(b=0.03)(b)$ Cyanobacteria and Total Sugar $(b=-0.001)(\mathbf{c})$ Alcaligenaceae and Threonine $(b=-0.001)(\mathbf{d})$ Porphyromonadaceae and Fruits and Vegetables $(b=-0.07)$. 

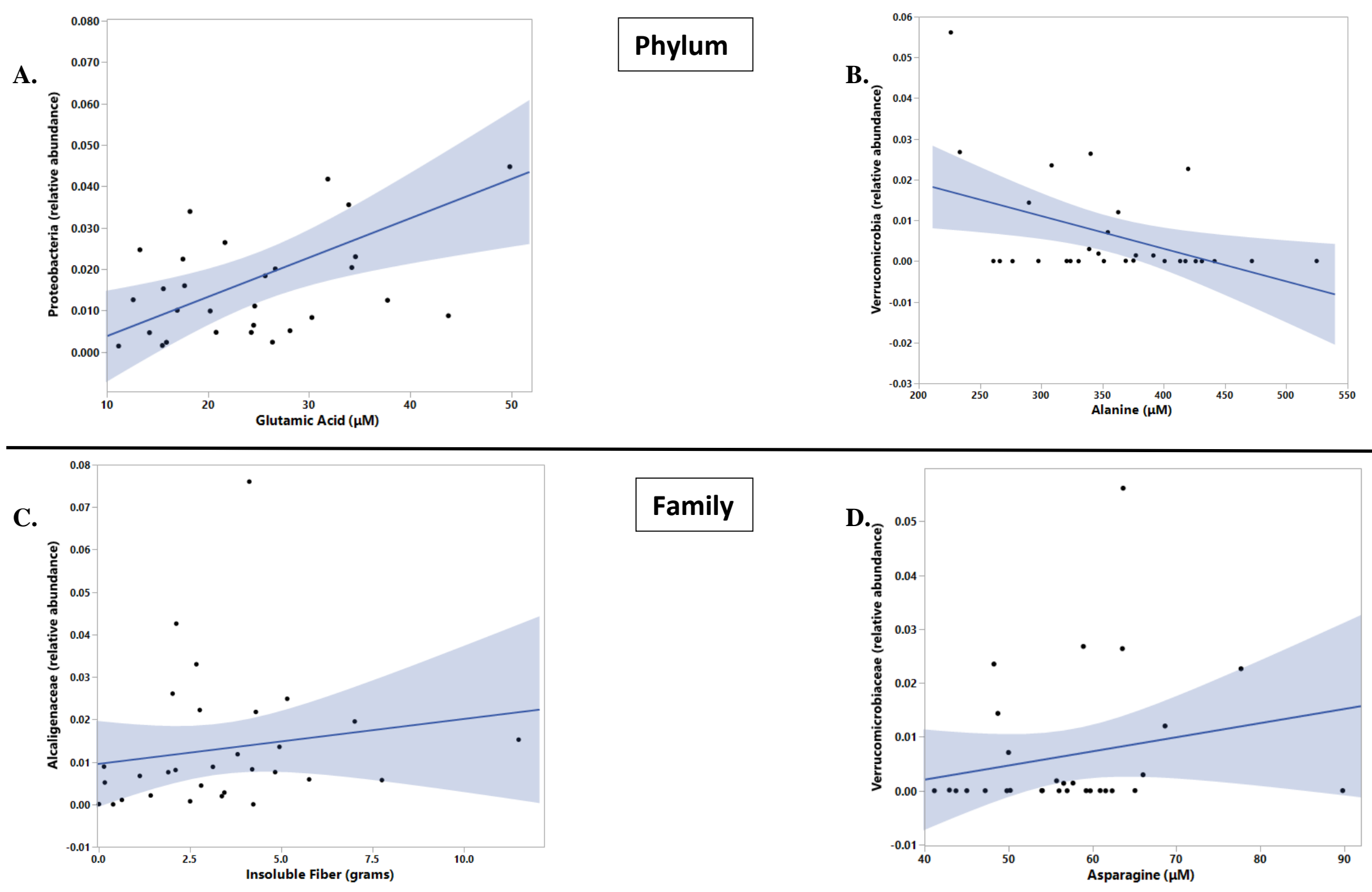

Figure 5: Post intervention significant stepwise regressions $(\mathrm{b}=$ slope $)$ among taxon relative abundance, dietary factors, amino acids factors. (a) Proteobacteria and Glutamic acid $(b=0.003)(\mathbf{b})$ Verrucomicrobia and Alanine $(b=-0.0005)$ (c) Alcaligenaceae and Insoluble Fiber $(b=0.05)(d)$ Verrucomicrobiaceae and Asparagine $(b=0.002)$. 
Conclusions, Implications, and Innovation 
The objective of the FRUVEDomics pilot study (Clinical Trial Record 1409433435) was to investigate how the implementation of the Dietary Guidelines for Americans in young adults with higher disease risk impacted food consumption and the dynamic environment of the microbiome as it relates to metabolic health. Not only did our tailored, theoretically driven, intervention significantly promote adaptation to a nutritionally dense diet in our study population, but it improved metabolic parameters and shifted microbial composition to one that can improve metabolic processes and decrease future risk of disease. The plexus surrounding diet and the microbiome is a research agenda that remains to be unraveled, however our strategy helped employ a realistic, and free-living, human study necessary to help clarify the likely realworld interaction. More importantly, the FRUVEDomics project drove the understanding of the systemic inflammatory response in young adults with higher risk of disease and helped identify metabolic markers that may be present for those at increased risk of other potential comorbidities. In gaining this knowledge, it will help generate more effective and personalized therapeutic interventions for young adults who are undergoing a transition of life where behavior is learned and lifestyle modification can be promoted for a sustainable future.

A novel approach that our group accomplished in this project was usage of a metabolomics core to understand the pathophysiology of poor health as it correlates with the microbiome. This integration of gene sequence of the microbiome and metabolome will pave the way toward a better molecular understanding of the complex mammalian superorganism. Lastly, our group is one of the first programs in Appalachia to investigate microbiome interactions, certain metabolites, as well as environmental and lifestyle factors in a high risk population. The use of these research findings from a team science initiative will improve clinical practices, 
disease prevention strategies, and public health policies. Altogether, this represents the first of many studies that will be needed in the development of precision medicine. 
Chapter IV: Addendum 


\title{
Metabolome Paper
}

\section{Efficacy of nutritional interventions to lower circulating ceramides in young adults at risk of developing the metabolic syndrome: FRUVEDomic study.}

Alice T. Mathews ${ }^{1}$, , Oluremi A. Famodu ${ }^{1}$, Melissa D. Olfert ${ }^{1}$, Pamela J. Murray ${ }^{2}$, Christopher F. Cuff ${ }^{3}$, Marianne T. Downes ${ }^{4}$, Norman J. Haughey ${ }^{5}$, Sarah E. Colby ${ }^{6}$, Paul D. Chantler ${ }^{7,8}$, I. Mark Olfert ${ }^{7,8}$, and Joseph W. McFadden ${ }^{1}$.

${ }^{1}$ Division of Animal and Nutritional Sciences, West Virginia University, Morgantown, West Virginia; ${ }^{2}$ Department of Pediatrics, West Virginia University School of Medicine, Morgantown, West Virginia; ${ }^{3}$ Department of Microbiology, Immunology, and Cell Biology, West Virginia University School of Medicine, Morgantown, West Virginia; ${ }^{4}$ Division of Medical Laboratory

Sciences, West Virginia University School of Medicine, Morgantown, West Virginia;

${ }^{5}$ Department of Neurology, Johns Hopkins University School of Medicine, Baltimore, Maryland;

${ }^{6}$ Department of Nutrition Science, University of Tennessee, Knoxville, Tennessee; ${ }^{7}$ Department of Exercise Physiology, West Virginia University School of Medicine, Morgantown, West Virginia; ${ }^{8}$ Cardiovascular \& Respiratory Research Group, West Virginia University School of Medicine, Morgantown, West Virginia.

*denotes equal contribution

\begin{abstract}
The 2010 USDA Dietary Guidelines for Americans (DGA) recommends a diet largely composed of fruit and vegetables. Consuming a diet high in fruit and vegetables, and low in refined carbohydrates and saturated fat may reduce an individual's risk for type 2 diabetes, nonalcoholic fatty liver disease, low-grade chronic inflammation, and metabolic syndrome (MetS). Several recent studies have implicated the bioactive sphingolipid ceramide as an associative and causative biomarker for the development of these conditions. Considering that the intake of fruit and vegetables is frequently inadequate in young adults, we performed a pilot investigation to assess the efficacy of a free-living fruit and vegetable intervention on overall metabolic health, circulating ceramide supply, and inflammatory status in young adults at variable risk for MetS. We discovered that adoption of the recommended DGA for fruit and vegetable intake for eight weeks decreased waist circumference, systolic blood pressure, circulating cholesterol, and alanine aminotransferase. Lipidomics analysis revealed that nutritional intervention can lower circulating ceramides and glycosylated ceramides, including C24:0 ceramide, a known inhibitor of insulin signaling. Unexpectedly, we observed an increase in C16:0 ceramide suggesting that this form of ceramide in circulation is not associated with metabolic disease. We also observed an improved inflammatory status with enhanced fruit and vegetable intake that was correlated with ceramide concentrations. Future research needs to assess whether the ceramide-lowering ability of nutritional intervention is associated with reduced risk of developing metabolic disease.
\end{abstract}

Keywords: ceramide, fruit and vegetable intervention, insulin resistance, metabolic syndrome 


\section{Introduction}

Inadequate intake of fruit and vegetables represents an unhealthy dietary behavior that results in disproportionally higher rates of obesity-related morbidity and mortality in young adults residing in the United States $[183,184]$. Recent evidence has demonstrated that the inadequate consumption of fruit and vegetables can predispose individuals to an increased risk of developing the metabolic syndrome (MetS), type 2 diabetes mellitus, and cardiovascular disease $[183,185-187]$. This elevated risk for metabolic disease is alarming if we consider that $78 \%$ of young adults consume less than the recommend amount of fruit and vegetables per day [24]. Epidemiological evidence indicates that the consumption of a diet enriched with fruit and vegetables, or one that is low in refined carbohydrates or saturated fats, can improve insulin sensitivity and lower circulating LDL-cholesterol, and may provide a simple approach to lower an individual's risk of developing chronic metabolic dysfunction [12, 188-190]. Given the clear link between inadequate nutritional intake and obesity-related diseases, evaluating the effectiveness of dietary interventions to restore healthy metabolic function is necessary.

An important mechanism that regulates the progression of insulin resistance and related metabolic disorders involves the enhanced synthesis of the bioactive sphingolipid ceramide. Findings from in vivo investigations have implicated ceramides in the development of the MetS in association with diabetic dyslipidemia, hepatic lipid deposition, and inflammation, as reviewed by Holland and Summers [191]. Available evidence suggests that ceramide regulates insulin signaling by preventing the translocation of glucose transporters to the plasma membrane through inactivation of PKB [192]. In addition to antagonizing insulin signaling, elevations of C24:0 ceramide in circulating lipoproteins have been demonstrated to increase levels of the inflammatory cytokine IL-6 and monocyte chemoattractant protein-1 (MCP-1), and to decrease 
anti-inflammatory IL-10 expression in macrophages [192]. Lowering ceramide concentrations by inhibiting synthesis improves insulin resistance, reduces hepatic lipid accumulation, and reduces inflammation associated with excess energy intake [193, 194]. Based on these data, ceramides have been recognized as potential biomarkers for insulin resistance, non-alcoholic fatty liver disease (NAFLD), and MetS [195, 196]; however, the identification of dietary recommendations to lower ceramide levels in humans has been underexplored.

Diets high in saturated fat increase hepatic ceramide synthesis and accumulation in rodents with impaired mitochondrial fatty acid processing and insulin sensitivity [194, 197], conditions that often develop in parallel with NAFLD [198]. Specifically, palmitic acid is partitioned away from oxidation and transformed into alternative metabolic fates including ceramide. Although much of the data demonstrating the associative or causative relationship between ceramide and insulin resistance has been derived from high-saturated fat feeding studies, Vilá et al. [199] have established that hepatic ceramides are increased in rats fed fructose-sweetened water. Vila and coworkers propose that the ability of fructose to promote ceramide synthesis may be attributed to impaired fatty acid $\beta$-oxidation [199]. Indeed, diets rich in refined carbohydrates such as those found in soft drinks can exacerbate NAFLD [200, 201] and therefore may potentially increase hepatic ceramide synthesis and export. We recognize that fruit and vegetables are sources of naturally occurring fructose; however, the replacement of dietary refined carbohydrates and saturated fat with fruit, and the increased consumption of beneficial phytochemicals and antioxidants likely maintain hepatic mitochondrial function and thus minimize hepatic ceramide synthesis and accumulation in blood.

Studies evaluating the efficacy of practical nutritional interventions to lower circulating ceramide in free-living subjects are needed. We hypothesize that a dietary intervention focused 
on increasing the intake of fruit and vegetables, and reducing the consumption of refined carbohydrates or fat in free-living subjects would lower serum ceramide concentrations in young adults 'at risk' for developing the MetS. Our pilot investigation targeted individuals that were between 18 and 28 years of age. Additionally, we focused our attention on palmitoyl (C16:0) and C24:0 ceramide because of their associative and causative role in the development of metabolic disease [192, 202-204].

\section{Methods}

The West Virginia University Institutional Review Board approved this research project that was initiated in January 2015. All participants provided written informed consent.

\section{Subject screening}

To evaluate eligibility for participation in the study, young adults in the surrounding area of West Virginia University (Morgantown, WV) were screened via phone by a registered dietitian nutritionist (RDN). Subjects were asked a series of questions in five categories: 1) demographics (age, willingness to follow nutritional guidelines, height, weight, nationality, and pregnancy status), 2) metabolic risk factors (blood pressure, blood glucose, body shape (apple, pear, or no distinctive shape), medications taken, and family history of disease), 3) current nutritional status (meal frequency, food choices, and level of alcohol consumption), 4) physical activity level (frequency and type of physically activity), and 5) psychological and behavioral (satisfaction with weight and body shape, and assessment of risk for development of an eating disorder, and smoking/tobacco use). Individuals were excluded from the study if they were not within the age range of 18 to 28 years, pregnant, exhibited an eating disorder, treated for drug or alcohol abuse within the past year, received psychiatric treatment within the previous year, had 
used antibiotics in the last three months, displayed a body mass index (BMI) of less than 18, or weighed less than $50 \mathrm{~kg}$.

In order to screen individuals for the study, researchers employed a three step approach. The first step included designing a tool called the Metabolic Syndrome Risk Score to categorize individuals into 'high', 'medium' or 'low' risk of developing the MetS. The MetS risk score evaluated the five components of the MetS (waist circumference, blood pressure, fasting glucose, and circulating triacylglycerol (TAG) and HDL-cholesterol), but also considered nutritional status, physical activity, ethnicity, family history, and current medication use. Second, the assessment tool was administered by telephone between a trained researcher and subject. After a score was generated, and inclusion and exclusion criteria were met, the participant was asked to visit the Human Performance Lab at West Virginia University to have his or her health and nutritional history reviewed. The final step involved a team-based discussion to select final subjects for participation.

\section{Intervention design}

Following subject screening, thirty-six young adults (mean $=23.2 \pm 2.5$ years of age, range $=18$ to 28 years of age) participated in the 8 -week free-living nutritional intervention. Although most individuals were randomly balanced into three dietary intervention groups, subjects residing together were assigned into the same dietary intervention to maximize adherence and compliance to the intervention. The dietary interventions were based on the United States Department of Agriculture (USDA) MyPlate Dietary Guidelines for Americans (DGA; [7]) and were defined as follows: $50 \%$ of their meal plate must consist of fruit and vegetables (FRUVED; equivalent to 2.5 or 3 cups of vegetables for women and men, respectively, and 2 cups of fruit daily for all individuals). All subjects participated in the 
FRUVED diet, however this intervention was divided into 3 groups: FRUVED $(\mathrm{n}=12)$, FRUVED plus low refined carbohydrates (FRUVED + LRC; $n=12$ ), or FRUVED plus low fat $($ FRUVED + LF; $n=12)$. Subjects that were allocated to the FRUVED + LRC intervention were counseled to consume low-glycemic index foods (e.g. reduced intake of white bread, crackers, cake, and sugar-sweetened beverages) and less than 3 oz. of refined carbohydrates, similar to reported guidelines [7]. For FRUVED + LF, subjects were advised to consume a low-fat diet, as recommended by the American Heart Association guidelines [205]. Subjects were instructed to consume $30 \%$ or less of caloric intake from total fat (equally divided into saturated, monounsaturated, and polyunsaturated fat), however the overall message conveyed was to choose low-fat, and lean sources of dairy and protein in accordance with the USDA DGA.

\section{Education and monitoring}

Enrolled subjects were required to attend a two-hour group class (one for each of the different diets) by a RDN just prior to the start of the intervention. Subjects were provided instruction in basic nutrition guidelines, Nutrition Facts Label reading, grocery shopping and budgeting for fruit and vegetable consumption, resources from local markets, as well as allowed and prohibited foods for their intervention. Materials were personalized for each specific diet to include sample menus and snack suggestions. All subjects were provided a culinary toolkit to secure success in fruit and vegetable preparation and planning of meals. The kit included knives, cutting board, peeler, can opener, measuring cups for dry and liquid ingredients, and plastic bags and containers for food storage. With the exception of diet, subjects were instructed to maintain pre-intervention lifestyle behaviors including physical activity and sleep patterns.

On a weekly basis, participants met with the RDN to be counseled on their intervention plan. The RDN examined dietary behaviors from weekly participant food logs, meal photos, and 
analysis of a 24 h dietary recall using Nutritionist Pro software (version 1.2, Nutritionist Pro, San Bruno, CA). By reviewing these measurements weekly, the RDN was able to counsel subjects on ways to further adhere to their assigned intervention while ensuring compliance. Apart from these assessments, the RDN and participant independently rated participant overall progress and adherence of assigned diet intervention on a continuum of 1-100 each week. This weekly rated scale (which considered physical activity, stress, sleep, and medication use) was averaged weekly starting at week 2 , and if the average was below 90 , individuals were excluded from analysis due to noncompliance to intervention. To help with compliance, monetary incentives were provided each week (following the completion of scheduled visitations with the RDN) to help off-set the potential and/or perception of greater food expense of routinely purchasing fresh fruit and vegetables.

\section{Blood sampling and anthropometric measurements}

Fasting blood was collected by venipuncture prior to the intervention, and at wk 2, 5, and 8 of intervention. Blood was allowed to coagulate at room temperature for $30 \mathrm{~min}$ and then centrifuged at $3,400 \times g$ for $10 \mathrm{~min}$ at $4^{\circ} \mathrm{C}$. Following centrifugation, serum was decanted and stored at $-80^{\circ} \mathrm{C}$ until analysis. The West Virginia University, Ruby Memorial Hospital Clinical laboratory provided basic chemistry analysis (i.e. electrolytes, fasting blood glucose, alkaline phosphate, aspartate and alanine aminotransferase (AST and ALT, respectively)), lipid panel (i.e. TAG, cholesterol, HDL, LDL, VLDL), and endocrine (i.e. insulin, high-sensitive C-reactive protein) on blood samples collected pre and post (wk 8) intervention. Weekly physical activity was measured subjectively with physical activity logs and the International Physical Activity Questionnaire (IPAQ) pre and post intervention. Physical activity was also measured objectively with accelerometers (GT3X; ActiGraph, Pensicola, FL). Anthropometric measurements were 
taken before and after intervention. Body fat was measured via BodPod (CosMed USA Inc., Chicago, IL), height by an electronic Stadiometer (SECA 213), weight via bioelectrical impedence digita scale (SECA 874), and waist, hip and neck circumferences measured by Gulick tape. All measurements were taken twice and averaged by a trained researcher.

\section{Cytokine measurements}

Circulating cytokine concentrations were measured from venous serum samples using a Mesoscale V-PLEX human pro-inflammatory panel 1 (\#K15049G; MesoScale Diagnostics, LLC., Gaithersburg, MD) and chemokine panel 1 (\#K15047G) assay kits according to manufacturer protocol on a SECTOR Imager 2400 instrument (MesoScale Diagnostics, LLC.). Each subject's samples were assayed in duplicate and then averaged for final analysis. The average coefficient of variation of the standard curves generated compared to the expected standard values was less than $10 \%$ for all measured cytokines. Minimum detection levels for cytokines were as follows: IL-6 1.31 pg/mL, IL-10 0.59 pg/mL, INF- $\gamma 4.09$ pg/mL, TNF- $\alpha 0.87$ pg/mL, MCP-1 0.06 pg/mL, MCP-4 1.51 pg/mL, macrophage-derived chemokine (MDC) 2.11 $\mathrm{pg} / \mathrm{mL}$, thymus- and activation-regulated chemokine (TARC) $0.14 \mathrm{pg} / \mathrm{mL}$, and eotaxin 1.70 $\mathrm{pg} / \mathrm{mL}$.

\section{Ceramide measurements}

Serum ceramide extraction was conducted using previously established methods that employ a modified Bligh and Dyer procedure including C12:0-ceramide as an internal standard (Avanti Polar Lipids, Alabaster, AL) [206, 207]. The organic layers from plasma extracts were dried in a nitrogen evaporator (Organomation Associates Inc., Berlin, MA) and re-suspended in pure methanol prior to analysis. Ceramides were detected by multiple reaction monitoring using a liquid chromatography coupled electrospray ionization tandem mass spectrometry 
(ESI/MS/MS; API3000; AB Sciex Inc., Thornhill, ON, Canada) operated in positive mode. Liquid chromatography and MS/MS parameters have been previously described [208]. Slight differences in extraction efficiency and fluctuations in mass spectrometer efficiency were normalized to the C12:0-ceramide internal standard. Instrument efficiency was monitored daily and individual plasma extracts were re-analyzed if the internal standard deviated more than $20 \%$ from the overall median internal standard value. The original or re-run data with internal standard concentrations closest to the median C12:0-ceramide value were included in the final analysis. Plasma ceramide concentrations were determined by fitting the identified ceramide species to standard curves based on acyl-chain length. Ceramide standards were purchased from Sigma-Aldrich (C16:0, C18:0, and C18:1; St. Louis, MO) or Avanti Polar Lipids (C20:0, C22:0, C24:0, and C24:1). Glycosylated ceramides were purchased from Matreya Inc. (C24:0-GlcCer, C16:0-LacCer, C18:0-LacCer; Pleasant Gap, PA). Instrument control and quantitation were performed using Analyst 1.4.2 and MultiQuant software (AB Sciex Inc.), respectively.

\section{Statistical analysis}

An analysis of variance (ANOVA) was used to examine difference among the groups. Anthropometric and plasma data were analyzed as repeated measures over time relative to start of intervention under the MIXED procedure of SAS (version 9.3; SAS Institute Inc.). The statistical model included the random effect of subject, and the fixed effects of intervention and week relative to start of intervention, and their interaction. The model included post hoc adjustment for multiple comparisons using Tukey's adjustment. The method of Kenward-Rogers was used for calculation of denominator degrees of freedom. Normality of the residuals was evaluated with normal probability, box plots, and homogeneity of variances with plots of residual versus predicted values. When necessary, data were transformed and presented in the 
original scale. When the intervention effect or intervention $\times$ week interaction were significant, the SLICE option of SAS was used to compare intervention differences at individual time points. Studentized residual values $>3.0$ or $<-3.0$ were considered outliers and removed from the analysis (typically 1 per response variable). Parametric Pearson correlations were performed in order to determine associations between plasma ceramides, anthropometric measurements, and circulating cytokines. All results are expressed as least squares means and their standard errors, unless stated otherwise. Significance was declared at $P \leq 0.05$, and trends toward significance were considered at $0.05<P \leq 0.10$.

\section{Results and Discussion}

The principal finding of our study is that young adults who are overweight (BMI between 25 and 30) and 'at-risk' of developing MetS show improved metabolic health following adoption of the USDA recommended DGA of consuming 50\% fruit and vegetables, as evidenced by reductions in circulating ceramide, biomarkers of inflammation, systolic blood pressure, and waist circumference. It is important to emphasize the current intervention was a 'free-living' study design permitting subjects to freely choose the type and amount of food they consumed within their own environment with the requirement that their meals meet the recommended $50 \%$ fruit and vegetable consumption. While this approach does not control total nutritional intake, the purpose of this study was not to induce weight loss per se, but rather determine if adopting the DGA for fruit and vegetables would produce beneficial clinical changes and/or changes in biomarkers linked with the progression of cardiometabolic disease.

Although thirty-six subjects participated, six subjects were not included within our final analyses because the average weekly compliance score fell below the a priori threshold. Therefore, our data only report the data from thirty compliant subjects with a mean MetS risk 
score of $4.1 \pm 0.3$ and a mean age of $23.6 \pm 0.4$ (range $=18$ to 28 years of age; $53 \%$ female; $66 \%$ Caucasian; Table 1). The moderate MetS risk score was primarily attributed to subjects being overweight $(\mathrm{BMI}=27.2 \pm 5.9$; Table 2$)$; albeit, subjects displayed normal concentrations of circulating fasting triacylglycerol, glucose and HDL-cholesterol, as well as normal systolic and diastolic blood pressure (Table 2 and 3). At study enrollment, demographics and MetS risk score were comparable for the FRUVED, FRUVED + LRC, and FRUVED + LF interventions; however, the spread of ethnicity was lower in the FRUVED group.

\section{Nutrient intake}

Prior to intervention, all compliant subjects consumed less than 5 cups/d of fruit and vegetables, as recommended by the 2010 USDA DGA (Table 4). Following the 8-wk intervention, all subjects exceeded recommendations by consuming $108 \%$ more fruit and vegetables $(P<0.001)$, compared to pre-enrollment. Moreover, intake of total empty calories (defined as calories from added sugar and added fat by DGA) and fat was reduced in all subjects by 56 and $8 \%$, respectively $(P<0.05)$. Regarding fat intake, the consumption of saturated fat was reduced by $8.3 \pm 1.5 \mathrm{~g}$ post-intervention $(P<0.05)$, whereas intake of unsaturated fat or cholesterol was not modified. In contrast, total fiber and sugar intake increased by 74 and 34\%, respectively $(P<0.05)$. Specifically, all subjects consumed more insoluble and soluble fiber as well as several sugars including glucose, fructose, and sucrose, relative to pre-intervention. Although nutrient intake was comparable across all interventions, FRUVED + LRC consumed less soluble and insoluble fiber, relative to FRUVED $+\operatorname{LF}(P<0.001)$. Although subjects were compliant, only a few differences in the measured dietary components were seen among the three dietary arms. Therefore, the data suggests that the FRUVED + LRC or FRUVED + LF did not modify intake of refined carbohydrates or fat, relative to FRUVED. The reason for no 
observed differences could be a limitation of using self-reported measurements of dietary intake or that our pilot study did not include a control group. Alternatively, consuming more fruit and vegetables may concomitantly decrease fat and processed carbohydrate intake.

\section{Assessment of metabolic health}

Although subjects were clinically healthy at the start of intervention, we observed a significant decrease in waist circumference and systolic blood pressure in all subjects by wk 8 ( 3 and $6 \%$, respectively; $P<0.01$; Table 2). We did not observe changes in body weight, BMI, body fat $\%$, hip and neck circumference, or diastolic blood pressure following intervention (Table 2). Epidemiological studies have demonstrated lower BMI with higher fruit and vegetable intake [185-187]; however, a decrease in adiposity is not always associated with this feeding behavior [184]. Contrasting anthropometric conclusions may be attributed to differences in total energy intake, duration of behavior, demographics, type of food, or method of food preparation. Because waist circumference and systolic blood pressure are strong risk factors for the development of MetS, type 2 diabetes and cardiovascular disease [189, 209], we consider the observed decreases in these measurements to be favorable intervention outcomes. Comparable to our anthropometric evaluation, we did not observe changes in glucose, insulin, estimated insulin sensitivity, LDL-cholesterol, VLDL-cholesterol, AST, or ALT (Table 3). However, we did detect lower circulating cholesterol by wk $8(5 \% ; P=0.05)$. In contrast, we observed a significant decrease in HDL-cholesterol $(P<0.001)$ post-intervention, where higher levels are associated with atheroprotective properties [210] and others have observed a similar HDLcholesterol response following a high fiber intervention [127]. To positively impact HDLcholesterol, researchers suggest replacing saturated fat with unsaturated fat instead of decreasing overall fat intake [132], which was not a component of our study design. 
Previous work has demonstrated an inverse relationship between the incidence of diabetes and the consumption of fruit and vegetables [183], a response that may be attributed to increased intake of dietary fiber, and decreased consumption of fat and refined carbohydrates as observed in our investigation. Of importance, our subjects were instructed not to change physical activity levels during the study in order to prevent confounding beneficial influences that could be conferred by exercise. Given the physical activity levels were found to not be different between pre versus post measures in all our subjects, we believe the improved metabolic health was due solely to change in nutrition.

\section{Modifications in circulating ceramides}

Current evidence suggests that the development of insulin resistance, NAFLD, and the MetS is intrinsically linked with the accumulation of ceramide [193, 211, 212]. Increased caloric intake from saturated fat can activate hepatic ceramide synthesis in overweight subjects [213, 214], and subsequently increase lipoprotein ceramide packaging and export [192]. In turn, circulating lipoprotein ceramide can mediate inflammatory cytokine release from adipose tissue macrophages, and inhibits insulin-stimulated PKB activation in skeletal muscle [192]. These proposed mechanisms are consistent with data from several studies that have reported elevated circulating ceramide in obese adolescents, adults with type 2 diabetes, or rodents with NAFLD [195, 213, 215]. Avoiding body weight gain, or consuming diets low in saturated fatty acids or fructose from non-fruit sources are a likely means to increase hepatic fatty acid oxidation, inhibit ceramide synthesis, and lower circulating ceramide to improve metabolic health [216, 217]. To assess this possibility, we employed a targeted lipidomic approach to quantify serum ceramides, GlcCer, and LacCer in young adults consuming a diet enriched with fruit and vegetables. 
The intake of a diet high in fruit and vegetables but low in refined carbohydrates and fat decreased circulating total ceramide concentrations by wk 5 in young adults (16 and $48 \%$ for FRUVED + LRC and FRUVED + LF, respectively; $P<0.05$; Figure 1). However, we did not observe a change in total ceramide levels in the FRUVED group. We also measured serum GlcCer, a glycosphingolipid formed via glycosylation of ceramide by the enzyme glucosylceramide synthase. We observed that serum total GlcCer concentrations were lower for all subjects by wk 8 (20, 32, and 46\% for FRUVED, FRUVED + LRC, and FRUVED + LF, respectively; $P<0.001$; Figure 1), relative to start of interventions. Similar to ceramide, GlcCer levels are elevated in overweight animals [194, 207]; however, the ability of circulating GlcCer to directly antagonize insulin resistance remains uncertain. To further evaluate glycosphingolipid biology in our subjects, we quantified serum LacCer that are formed by a (1-4)- $\beta$ linkage of galactose to GlcCer. In our investigation, serum LacCer levels were not modified by intervention (Figure 1). Recent evidence suggests that non-glycosylated ceramides are stronger circulating biomarkers for diabetes or prediabetes in adults as compared with GlcCer or LacCer [196]. However, both ceramides and GlcCer have been implicated in the pathogenesis of insulin resistance at the cellular level [194].

The majority of circulating ceramides are believed to be found within liver-derived lipoproteins in humans $[192,218]$. Previous research has confirmed that C24:0 ceramide is the most abundant ceramide in circulation [213], a subspecies produced by hepatic ceramide synthase 2 [219]. Indeed, we observed that C24:0 ceramide and C24:0 GlcCer were highly abundant in serum, representing $77 \%$ of total ceramide and $36 \%$ of total GlcCer at the initiation of intervention (Figure 2). Relative to C24:0 ceramide, we detected low circulating levels of 
C16:0 ceramide ( $0.9 \%$ of total ceramide) which is synthesized by ceramide synthase 6 . In contrast, C16:0 LacCer was the most abundant serum LacCer.

We discovered that the majority of circulating ceramides decreased with time for FRUVED + LRC and FRUVED + LF interventions (Figure 3); albeit the response was often greater for FRUVED + LF. For instance, the concentrations of C22:0, C24:1, and C26:0 ceramide were significantly lower by wk 5 of intervention ( 29 to $64 \%$ for FRUVED + LRC and FRUVED + LF; $P<0.05$; Figures 3 and 4); ceramide moieties associated within increased BMI and fasting blood glucose in adolescents with type 2 diabetes [195]. Most notably, subjects following a diet containing high fruit and vegetables with low dietary fat displayed a $50 \%$ decrease in C24:0 ceramide by wk 5, relative to the start of intervention $(P<0.01$; Figure 4$)$. We view the lowering of C24:0 ceramide concentration in serum as a favorable intervention outcome because lipoprotein $\mathrm{C} 24: 0$ ceramide inhibits $\mathrm{PKB}$ activation and glucose transporter translocation in skeletal muscle [192], and circulating C24:0 ceramide is inversely related to insulin-stimulated glucose disposal in humans [196]. High dietary intake of saturated palmitic acid can increase C24:0 ceramide accrual [220]. Although we did not detect a change in total or saturated fat intake for FRUVED + LF, relative to FRUVED, the FRUVED + LF group may have exhibited a lower intake of palmitic acid, a fatty acid required for de novo ceramide synthesis. Alternatively, hepatic palmityl-CoA supply may have been limited in these subjects because of suppressed de novo lipogenesis or enhanced mitochondrial oxidation. These possibilities may also explain the ceramide-lowering ability of FRUVED + LRC. Moreover, differences in circulating and liver fatty acid pools may explain why FRUVED alone was unable to lower ceramide. 
Although the majority of circulating ceramides declined following intervention, we did observe an 80,70, and 50\% increase in circulating C16:0 ceramide by wk 8 for FRUVED, FRUVED + LRC, and FRUVED + LF $(P<0.05$; Figure 4). Furthermore, the ratio of C16:0 to C24:0 ceramide increased 66\% across time $(P<0.01$; Figure 5$)$. We and others have previously observed a reciprocal relationship between circulating C16:0 and C24:0 ceramide [207]. Researchers that have recently evaluated tissue ceramide supply and insulin action have proposed that $\mathrm{C} 16: 0$ ceramide is a proapoptotic mediator of the pathophysiology of insulin resistance in response to a high saturated fat diet, and in contrast, C24:0 ceramide confers antiapoptotic and proliferative functions [203, 204]. Nevertheless, our measured increase in serum C16:0 ceramide that developed concurrently with lower circulating cholesterol and reduced waist circumference is a paradoxical observation. In support of our findings, Meikle et al. did not observe a relationship between circulating C16:0 ceramide and type 2 diabetes [196]. The ability of C16:0 ceramide to antagonize insulin action may be reliant upon the intracellular tissue concentration of this sphingolipid which was not characterized in our investigation. Additionally, the ability of extracellular lipoprotein ceramide to antagonize insulin signaling may involve a different mode of action since long-chain ceramides are highly hydrophobic and cannot pass freely through the plasma membrane [221]. The relationship between the acyl chain length of circulating ceramide and insulin-stimulated glucose disposal requires further investigation to definitively distinguish $\mathrm{C} 16: 0$ or $\mathrm{C} 24: 0$ ceramide as the causal agent of insulin resistance in humans; thereafter, nutritional interventions aimed at minimize ceramide synthesis and metabolic disease can be further refined.

Changes in serum GlcCer were similar to the supply of ceramide (Figure 3 and 6). Relative to the start of each intervention, we observed a 31 to $73 \%$ decrease in serum C22:0, 
C24:0, and C24:1 GlcCer by wk 5 in FRUVED + LRC and FRUVED + LF subjects $(P<0.05)$, respectively. In contrast, intervention did not modify serum C16:0, C20:0, and C26:1 GlcCer levels. For LacCer sphingolipids, we detected a decrease in C22:0 and C24:0 LacCer with time (17 and 37\% decrease by wk 5; $P<0.01$; Figure 3 and 7); however, we did not observe changes in the concentrations of serum C16:0, C24:1, or C26:1 LacCer with intervention. Furthermore, we observed a strong positive association between C24:0 ceramide and GlcCer, and between C24:0 GlcCer and LacCer across all sampled subjects (Figure 8). These results may indicate a coordinated regulation of synthesis for glycosphingolipids with the C24:0 acyl moiety. Although glycosylated ceramides may mediate the link between obesity and insulin resistance [222], the causal role of glycosphingolipids in the progression of insulin resistance is actively being defined. Current evidence suggests that the insulin receptor is co-localized with glycosphingolipids in plasma membrane caveolae [223], and excessive ganglioside content can disrupt insulin signaling by displacing the insulin receptor from these microdomains [224]. Modifying dietary energy intake is a likely means to control glycosylated ceramide supply. For instance, mice fed a low fat diet exhibit lower circulating C20:0, C22:0, and C24:0 GlcCer levels as compared with mice fed a high fat diet [225]. It is important to recognize that the observed ceramide and GlcCer-lowering response associated with increased fruit and vegetable consumptions developed even though dietary fructose intake increased. Therefore, the ability of dietary fructose to augment ceramide synthesis likely depends on compromised hepatic function which was not evident in our investigation as demonstrated by clinically low concentrations of circulating triacylglycerol, VLDL-cholesterol, AST, and ALT, as supported by Vila and coworkers [199]. 


\section{Inflammation and ceramides}

The development of the MetS or type 2 diabetes are recognized inflammatory conditions in overweight individuals. Specifically, increased adiposity can enhance the release of TNF- $\alpha$, IL-6, MCP, and other products of macrophages that populate adipose tissue. As a consequence, these pro-inflammatory cues interfere with insulin action by suppressing insulin signal transduction [226]. Consuming a diet rich in fiber and monounsaturated fat while reducing intake of saturated fat and cholesterol can increase fat expenditure and reduce low-grade chronic inflammation [227]; therefore, we assessed the inflammatory status of subjects at enrollment and wk 8 of intervention. Although we did not detect changes in circulating cytokines for specific diet regimens, a fruit and vegetable intervention lowered circulating MCP-4 and TARC in all subjects by wk $8(P<0.05$; Table 5$)$. We also observed a tendency for serum anti-inflammatory IL-10 concentration to increase $(P=0.06)$; however, a fruit and vegetable intervention was unable to modify inflammatory IL-6, TNF- $\alpha$, MCP-1, eotaxin, or INF- $\gamma$ levels. The ability of fruit and vegetable intake to suppress inflammation may be due to bioactive nutrients found in these foods including folate, flavonoids, and antioxidants [13, 228]. Specifically, the consumption of fruit or vegetables, or the intake of vitamin $\mathrm{C}$ and $\beta$-carotene is inversely associated with pro-inflammatory markers in adolescents [229].

The ability of inflammation to increase the synthesis of ceramide which can then antagonize insulin action has been documented $[192,214]$. For instance, saturated fatty acids can activate proinflammatory receptor toll-like receptor 4 which increases the expression of serine palmitoyltransferase and ceramide synthase in skeletal muscle, and activate hepatic sphingomyelinase [214]. In our study, multiple ceramides were positively correlated with proinflammatory cytokines (Table 6). For example, C20:0, C22:0, and C22:1 ceramide were 
positively associated with MDC, TARC, TNF- $\alpha$, and eotaxin $(\mathrm{r}=0.33$ to $0.54 ; P<0.05)$. A similar relationship was observed for a limited number of GlcCer or LacCer subspecies. Although the majority of sphingolipids measured were not correlated with INF- $\gamma$ or IL-10, highly abundant C24:0 ceramide and GlcCer were significantly correlated with serum INF- $\gamma$ and IL-10 ( $\mathrm{r}=0.34$ and $-0.35 ; P=0.05)$, respectively. Interestingly, the inhibition of ceramide synthesis can negate the antagonistic effects of inflammation on insulin sensitivity [214]; therefore, dietary interventions designed to lower circulating ceramide may be a means to improve insulin sensitivity in overweight subjects experiencing inflammation.

\section{Conclusion}

In summary, our pilot study demonstrates that an 8-wk free-living fruit and vegetable intervention designed according to the USDA MyPlate guidelines, known as the DGA, can lower waist circumference, reduce systolic blood pressure, and decrease circulating cholesterol in young adults at moderate risk for developing the MetS. Moreover, we establish that a short-term nutritional intervention can lower ceramide, a biomarker for metabolic disease. Of particular interest, we observed lower levels of circulating C24:0 ceramide with intervention which is a known antagonist of insulin action. Although the majority of ceramides were lowered by the study intervention, we unexpectedly observed an increase in serum C16:0 ceramide raising the possibility that circulating C16:0 ceramide may not be the causal agent of insulin resistance in humans. In addition to the changes in ceramide supply, a fruit and vegetable intervention lowered circulating inflammatory cytokines. Collectively, these data suggest that consuming a healthy diet improves clinical indices and biomarkers of metabolic and cardiovascular health, irrespective of changes in body mass. Future work with a larger sample size is needed to better understand the effects of macro or micronutrients on specific species of ceramides, and the 
mechanisms by which these ceramides exert their action, as well as their etiology in the development of obesity and chronic diseases such as the MetS, diabetes and cardiovascular disease. 


\section{Tables/Figures}

Table 1. Demographics and metabolic syndrome (MetS) risk score for compliant intervention subjects. ${ }^{1}$

\begin{tabular}{rllll}
\hline & $\begin{array}{c}\text { All subjects } \\
\mathrm{n}=30\end{array}$ & $\begin{array}{c}\text { FRUVED } \\
\mathrm{n}=12\end{array}$ & $\begin{array}{c}\text { FRUVED + LRC } \\
\mathrm{n}=8\end{array}$ & $\begin{array}{c}\text { FRUVED + LF } \\
\mathrm{n}=10\end{array}$ \\
\hline MetS risk score & $4.1 \pm 0.3$ & $4.4 \pm 0.5$ & $4.0 \pm 0.6$ & $3.8 \pm 0.5$ \\
Age, years & $23.6 \pm 0.4$ & $23.7 \pm 0.4$ & $22.1 \pm 1.2$ & $24.8 \pm 0.6$ \\
Female & $16(53)$ & $7(58)$ & $5(62)$ & $6(60)$ \\
Male & $14(47)$ & $5(42)$ & $3(38)$ & $4(40)$ \\
White & $20(66)$ & $10(83)$ & $3(38)$ & $7(70)$ \\
African American & $2(7)$ & 0 & $2(25)$ & 0 \\
Hispanic & $3(10)$ & 0 & $2(25)$ & $1(10)$ \\
Asian & $3(10)$ & $1(8)$ & $1(12)$ & $1(10)$ \\
Other & $2(7)$ & $1(8)$ & 0 & $1(10)$ \\
Appalachian & $18(80)$ & $9(75)$ & $4(50)$ & $5(50)$ \\
Non Appalachian & $12(40)$ & $3(25)$ & $4(50)$ & $5(50)$ \\
\hline
\end{tabular}

${ }^{1}$ Dietary interventions based on the United States Department of Agriculture MyPlate Dietary Guidelines for Americans included fruit and vegetables (FRUVED), FRUVED plus low refined carbohydrates (FRUVED + LRC), or FRUVED plus low fat (FRUVED + LF). Values within parenthesis represent percent of $n$.

${ }^{2}$ Cumulative score determined by the five components of the MetS (waist circumference, blood pressure, fasting glucose, and circulating triacylglycerol and HDL-cholesterol), as well as nutritional status, physical activity, ethnicity, family history, and current medication use. Scores ranged from 1 to 7 on a 12-point scale. Increased scores are indicative of increased risk for developing the MetS. 
Table 2. Anthropometric and blood pressure measurements before and after intervention. ${ }^{1}$

\begin{tabular}{|c|c|c|c|c|c|c|}
\hline & \multicolumn{2}{|c|}{ All subjects $(\mathrm{N}=30)$} & \multirow[b]{2}{*}{ SEM } & \multicolumn{3}{|c|}{$P$-value } \\
\hline & Pre & Post & & Group & Time & Group $\times$ Time \\
\hline Weight, kg & $77.0 \pm 15.1$ & $76.0 \pm 14.5$ & & 0.49 & 0.07 & 0.48 \\
\hline BMI, $\mathrm{kg} / \mathrm{m}^{2}$ & $26.4 \pm 4.2$ & $26.0 \pm 4.2$ & & 0.33 & 0.05 & 0.88 \\
\hline Body fat, $\%$ & $28.6 \pm 14.3$ & $25.9 \pm 10.7$ & & 0.60 & 0.14 & 0.37 \\
\hline Waist circumference, $\mathrm{cm}$ & $83.4 \pm 11.7$ & $80.6 \pm 10.4$ & & 0.76 & $<0.0001$ & 0.54 \\
\hline Hip circumference, $\mathrm{cm}$ & $102.9 \pm 9.8$ & $102.2 \pm 8.8$ & & 0.36 & 0.14 & 0.36 \\
\hline Neck circumference, $\mathrm{cm}$ & $36.0 \pm 3.9$ & $35.6 \pm 3.5$ & & 0.97 & 0.01 & 0.47 \\
\hline Systolic pressure, $\mathrm{mm} / \mathrm{Hg}$ & $118.6 \pm 15.3$ & $111.8 \pm 9.7$ & & 0.39 & 0.004 & 0.57 \\
\hline Diastolic pressure, $\mathrm{mm} / \mathrm{Hg}$ & $62.7 \pm 9.3$ & $60.3 \pm 7.2$ & & 0.36 & 0.12 & 0.76 \\
\hline
\end{tabular}

${ }^{1}$ Dietary interventions based on the United States Department of Agriculture MyPlate Dietary Guidelines for Americans included fruit and vegetables (FRUVED; $n=12$ ), FRUVED plus low refined carbohydrates (FRUVED + LRC; $\mathrm{n}=8$ ), or FRUVED plus low fat (FRUVED + LF; $\mathrm{n}=10$ ). Anthropometric measures were collected from all individuals at baseline and post intervention. Repeated Measures ANOVA was used to analyze the main effects of group, time, and their interaction. Values are presented as least squared means \pm SEM pre (wk 0 ) and post (wk 8) intervention.

${ }^{2}$ Body mass index. 
Table 3. Serum metabolic health measurements before and after intervention. ${ }^{1}$

\begin{tabular}{|c|c|c|c|c|c|}
\hline & \multicolumn{2}{|c|}{ All subjects $(\mathrm{N}=30)$} & \multirow{2}{*}{$\begin{array}{c}\text { Group Effect } \\
\text { P-Value } \\
\end{array}$} & \multirow{2}{*}{$\frac{\text { Time Effect }}{\text { P-Value }}$} & \multirow{2}{*}{$\frac{\text { Group } \times \text { Time Effect }}{\text { P-Value }}$} \\
\hline & Pre & Post & & & \\
\hline Glucose, $\mathrm{mg} / \mathrm{dL}$ & $86.9 \pm 7.5$ & $87.1 \pm 9.3$ & 0.29 & 0.87 & 0.17 \\
\hline Triglycerides, $\mathrm{mg} / \mathrm{dL}$ & $92.7 \pm 31.9$ & $87.9 \pm 30.4$ & 0.77 & 0.24 & 0.23 \\
\hline Cholesterol, mg/dL & $181.0 \pm 29.1$ & $171.7 \pm 29.3$ & 0.55 & 0.05 & 0.67 \\
\hline HDL-cholesterol, mg/dL & $56.6 \pm 12.4$ & $50.3 \pm 10.4$ & 0.96 & $<0.001$ & 0.50 \\
\hline LDL-cholesterol, mg/dL & $105.7 \pm 22.9$ & $103.7 \pm 23.9$ & 0.42 & 0.66 & 0.41 \\
\hline VLDL-cholesterol, mg/dL & $18.6 \pm 1.2$ & $17.7 \pm 1.1$ & 0.76 & 0.25 & 0.21 \\
\hline $\mathrm{AST}, \mathrm{U} / \mathrm{L}^{2}$ & $25.4 \pm 2.9$ & $22.7 \pm 1.9$ & 0.42 & 0.31 & 0.09 \\
\hline $\mathrm{ALT}, \mathrm{U} / \mathrm{L}^{3}$ & $28.7 \pm 4.9$ & $25.4 \pm 5.6$ & 0.31 & 0.24 & 0.81 \\
\hline Insulin $\mu \mathrm{IU} / \mathrm{mL}$ & $7.0 \pm 3.3$ & $7.4 \pm 4.1$ & 0.18 & 0.82 & 0.12 \\
\hline HOMA-IR $^{4}$ & $1.5 \pm 0.7$ & $1.6 \pm 0.9$ & 0.10 & 0.82 & 0.08 \\
\hline $\mathrm{Na}, \mathrm{mmol} / \mathrm{L}$ & $139.1 \pm 1.5$ & $138.2 \pm 3.2$ & 0.30 & 0.10 & 0.57 \\
\hline $\mathrm{K}, \mathrm{mmol} / \mathrm{L}$ & $3.8 \pm 0.3$ & $4.0 \pm 0.2$ & 0.72 & 0.006 & 0.86 \\
\hline
\end{tabular}

${ }^{1}$ Dietary interventions based on the United States Department of Agriculture MyPlate Dietary Guidelines for Americans included fruit and vegetables (FRUVED; $\mathrm{n}=12$ ), FRUVED plus low refined carbohydrates (FRUVED + LRC; $\mathrm{n}=8$ ), or FRUVED plus low fat (FRUVED + $\mathrm{LF} ; \mathrm{n}=10$ ). Biochemical measures were collected from all individuals at baseline and post intervention. Repeated Measures ANOVA was used to analyze the main effects of group, time, and their interaction. Significant effects ANOVA were followed by multiple comparisons using Tukey adjustment. Values are presented as least squared means \pm SEM pre (wk 0) and post (wk 8) intervention.

${ }^{2}$ Aspartate aminotransferase.

${ }^{3}$ Alanine aminotransferase.

${ }^{4}$ Homeostasis model assessment-estimated insulin resistance. Lower values are indicative of improved insulin sensitivity. 
Table 4. Intervention intake assessment. ${ }^{1}$

\begin{tabular}{|c|c|c|c|c|c|}
\hline & \multicolumn{2}{|c|}{ All subjects $(\mathrm{N}=30)$} & \multirow{2}{*}{$\frac{\text { Group Effect }}{\text { P-Value }}$} & \multirow{2}{*}{$\frac{\text { Time Effect }}{\text { P-Value }}$} & \multirow{2}{*}{$\frac{\text { Group } \times \text { Time Effect }}{\text { P-Value }}$} \\
\hline & Pre & Post & & & \\
\hline Total Calories, Kcal/d & $2263.3 \pm 375.1$ & $1935.7 \pm 622.6$ & 0.96 & 0.17 & 0.59 \\
\hline Empty Calories, Kcal/d ${ }^{2}$ & $997.6 \pm 948.1$ & $441.6 \pm 240.1$ & 0.54 & $<0.001$ & 0.87 \\
\hline Fruit and vegetables, cups/d & $2.5 \pm 2.0$ & $5.2 \pm 2.4$ & 0.70 & $<0.001$ & 0.51 \\
\hline Total Carbohydrates, g & $228.3 \pm 24.1$ & $259.6 \pm 16.5$ & 0.81 & 0.17 & 0.64 \\
\hline Total fiber, $\mathrm{g}$ & $19.2 \pm 12.6$ & $34.0 \pm 15.2$ & 0.12 & $<0.001$ & 0.95 \\
\hline Insoluble Fiber, $\mathrm{g}$ & $1.4 \pm 2.1$ & $3.3 \pm 2.5$ & $<0.001$ & 0.002 & 0.71 \\
\hline Soluble Fiber, g & $0.3 \pm 0.4$ & $0.8 \pm 0.6$ & 0.003 & $<0.0001$ & 0.82 \\
\hline Total sugar, g & $78.7 \pm 34.0$ & $105.4 \pm 43.5$ & 0.96 & 0.03 & 0.78 \\
\hline Glucose, $\mathrm{g}$ & $7.8 \pm 1.6$ & $15.3 \pm 1.6$ & 0.24 & $<0.001$ & 0.31 \\
\hline Fructose, $\mathrm{g}$ & $8.5 \pm 1.7$ & $16.8 \pm 1.7$ & 0.57 & $<0.001$ & 0.30 \\
\hline Galactose, $\mathrm{g}$ & $0.1 \pm 0.03$ & $1.1 \pm 0.6$ & 0.44 & 0.05 & 0.96 \\
\hline Sucrose, $\mathrm{g}$ & $10.1 \pm 2.03$ & $14.2 \pm 2.2$ & 0.13 & 0.003 & 0.79 \\
\hline Lactose, $\mathrm{g}$ & $6.2 \pm 1.9$ & $6.3 \pm 2.0$ & 0.14 & 0.31 & 0.86 \\
\hline Maltose, $\mathrm{g}$ & $0.5 \pm 0.2$ & $0.7 \pm 0.2$ & 0.23 & 0.01 & 0.94 \\
\hline Total Protein, g & $90.9 \pm 9.6$ & $91.4 \pm 6.2$ & 0.58 & 0.92 & 0.39 \\
\hline Total Fat, g & $90.1 \pm 10.8$ & $64.7 \pm 5.9$ & 0.84 & 0.003 & 0.36 \\
\hline Saturated fat, $g$ & $28.8 \pm 21.6$ & $20.5 \pm 13.1$ & 0.60 & 0.04 & 0.20 \\
\hline Monounsaturated fat, $g$ & $21.0 \pm 23.0$ & $16.8 \pm 12.0$ & 0.59 & 0.73 & 0.19 \\
\hline Polyunsaturated fat, $g$ & $13.9 \pm 15.0$ & $10.1 \pm 6.7$ & 0.44 & 0.67 & 0.60 \\
\hline Cholesterol, mg & $227.3 \pm 215.6$ & $247.4 \pm 247.5$ & 0.18 & 0.98 & 0.67 \\
\hline
\end{tabular}

${ }^{1}$ Dietary interventions based on the United States Department of Agriculture MyPlate Dietary Guidelines for Americans included fruit and vegetables (FRUVED; $\mathrm{n}=12$ ), FRUVED plus low refined carbohydrates (FRUVED + LRC; $\mathrm{n}=8$ ), or FRUVED plus low fat (FRUVED + LF; $n=10$ ). Dietary factors were collected and analyzed for main effects of group, time, and their interaction using Repeated Measures ANOVA. Significant effects ANOVA were followed by multiple comparisons using Tukey adjustment. Values are presented as least squared means \pm SEM at pre (wk 0) and post (wk 8) intervention.

${ }^{2}$ Added calories from fat and sugar. 
Table 5. Serum cytokine measurements before and after intervention. ${ }^{1}$

\begin{tabular}{llllll}
\hline & \multicolumn{2}{c}{ All subjects $(\mathrm{N}=30)$} & \multicolumn{2}{c}{ Group Effect } & \multicolumn{2}{c}{ Time Effect } & \multicolumn{2}{c}{ Group $\times$ Time Effect } \\
\cline { 2 - 6 } & \multicolumn{1}{c}{ Pre } & \multicolumn{1}{c}{ P-Value } & P-Value & P-Value & P-Value \\
\hline IL-6 & $4.1 \pm 0.6$ & $4.5 \pm 0.4$ & 0.59 & 0.39 & 0.99 \\
IL-10 & $2.1 \pm 0.5$ & $2.4 \pm 0.5$ & 0.84 & 0.06 & 0.84 \\
IFN- $\gamma$ & $23.5 \pm 4.9$ & $19.7 \pm 3.8$ & 0.24 & 0.84 & 0.93 \\
TNF- $\alpha$ & $10.5 \pm 0.5$ & $10.3 \pm 0.6$ & 0.51 & 0.19 & 0.46 \\
MCP-1 & $245.7 \pm 39.4$ & $207.2 \pm 23.5$ & 0.52 & 0.26 & 0.54 \\
MCP-4 & $152.3 \pm 39.4$ & $89.7 \pm 12.9$ & 0.44 & 0.02 & 0.11 \\
MDC $^{3}$ & $1299.7 \pm 140.1$ & $1176.6 \pm 138.1$ & 0.38 & 0.08 & 0.36 \\
TARC $^{4}$ & $222.9 \pm 38.3$ & $167.0 \pm 36.7$ & 0.31 & 0.04 & 0.71 \\
Eotaxin & $126.6 \pm 21.4$ & $99.9 \pm 16.0$ & 0.86 & 0.34 & 0.30 \\
\hline
\end{tabular}

${ }^{1}$ Dietary interventions based on the United States Department of Agriculture MyPlate Dietary Guidelines for Americans included fruit and vegetables (FRUVED; $\mathrm{n}=12$ ), FRUVED plus low refined carbohydrates (FRUVED + LRC; $\mathrm{n}=8$ ), or FRUVED plus low fat (FRUVED + LF; $\mathrm{n}=10$ ). Serum cytokines measures were collected from all individuals at baseline and post intervention. Repeated Measures ANOVA was used to analyze the main effects of group, time, and their interaction. Values are presented as least squared means \pm SEM pre (wk 0 ) and post (wk 8) intervention.

${ }^{2} \mathrm{MCP}$, monocyte chemoattractant protein.

${ }^{3} \mathrm{MDC}$, macrophage-derived chemokine.

${ }^{4} \mathrm{TARC}$, thymus- and activation-regulated chemokine. 
Table 6. Correlations between cytokines and ceramides. ${ }^{1}$

\begin{tabular}{|c|c|c|c|c|c|c|c|c|c|c|c|c|c|c|c|c|c|c|c|}
\hline \multirow{2}{*}{$\begin{array}{l}\mathrm{P} \\
\mathrm{e}\end{array}$} & & \multicolumn{2}{|c|}{ IL-6 } & \multicolumn{2}{|c|}{ IL-10 } & \multicolumn{2}{|c|}{ IFN- $\gamma$} & \multicolumn{2}{|c|}{$\mathrm{TNF}-\alpha$} & \multicolumn{2}{|c|}{$\mathrm{MCP}_{-1}{ }^{2}$} & \multicolumn{2}{|c|}{ MCP-4 } & \multicolumn{2}{|c|}{$\mathrm{MDC}^{3}$} & \multicolumn{2}{|c|}{$\mathrm{TARC}^{4}$} & \multicolumn{2}{|c|}{ Eotaxin } \\
\hline & & $\mathrm{r}$ & $P$ & $\mathrm{r}$ & $P$ & $\mathrm{r}$ & $P$ & $\mathrm{r}$ & $\mathrm{P}$ & $\mathrm{r}$ & $P$ & $\mathrm{r}$ & $P$ & $\mathrm{r}$ & $P$ & $\mathrm{r}$ & $\mathrm{P}$ & $\mathrm{r}$ & $\mathrm{P}$ \\
\hline \multicolumn{20}{|c|}{ a Ceramide } \\
\hline $\mathrm{r}$ & $\mathrm{C} 16: 0$ & -0.03 & 0.85 & -0.03 & 0.85 & 0.06 & 0.73 & -0.11 & 0.53 & -0.05 & 0.77 & -0.04 & 0.83 & 0.01 & 0.91 & -0.08 & 0.67 & -0.01 & 0.93 \\
\hline $\mathrm{s}$ & C20:0 & 0.29 & 0.10 & -0.09 & 0.62 & 0.05 & 0.78 & 0.33 & 0.07 & 0.09 & 0.61 & -0.03 & 0.83 & 0.36 & 0.04 & 0.34 & 0.05 & 0.40 & 0.02 \\
\hline o & $\mathrm{C} 22: 0$ & 0.27 & 0.13 & -0.20 & 0.26 & 0.17 & 0.34 & 0.34 & 0.06 & 0.16 & 0.38 & -0.07 & 0.69 & 0.36 & 0.04 & 0.40 & 0.02 & 0.42 & 0.01 \\
\hline $\mathrm{n}$ & $\mathrm{C} 22: 1$ & 0.26 & 0.15 & -0.14 & 0.43 & 0.28 & 0.13 & 0.41 & 0.02 & -0.02 & 0.89 & -0.11 & 0.55 & 0.43 & 0.01 & 0.51 & 0.01 & 0.54 & 0.01 \\
\hline 五 & $\mathrm{C} 24: 0$ & 0.21 & 0.25 & -0.01 & 0.97 & 0.34 & 0.05 & 0.29 & 0.11 & 0.19 & 0.31 & -0.03 & 0.85 & 0.29 & 0.11 & 0.26 & 0.15 & 0.27 & 0.13 \\
\hline , & C24:1 & 0.06 & 0.72 & -0.28 & 0.13 & 0.08 & 0.64 & 0.39 & 0.02 & -0.09 & 0.61 & -0.22 & 0.23 & 0.26 & 0.16 & 0.35 & 0.05 & 0.36 & 0.04 \\
\hline \multirow[t]{3}{*}{$\mathrm{S}$} & GlcCer & & & & & & & & & & & & & & & & & & \\
\hline & C20:0 & 0.29 & 0.10 & -0.27 & 0.14 & 0.09 & 0.62 & 0.31 & 0.08 & 0.15 & 0.41 & 0.01 & 0.94 & 0.31 & 0.09 & 0.29 & 0.11 & 0.40 & 0.02 \\
\hline & C22:0 & 0.27 & 0.13 & -0.29 & 0.11 & 0.27 & 0.13 & 0.27 & 0.14 & 0.29 & 0.11 & 0.02 & 0.91 & 0.26 & 0.15 & 0.30 & 0.09 & 0.37 & 0.04 \\
\hline o & $\mathrm{C} 24: 0$ & 0.21 & 0.25 & -0.35 & 0.05 & 0.28 & 0.12 & 0.25 & 0.16 & 0.29 & 0.11 & -0.01 & 0.93 & 0.20 & 0.28 & 0.29 & 0.11 & 0.36 & 0.05 \\
\hline \multicolumn{20}{|c|}{ accer } \\
\hline$r$ & C22:0 & 0.32 & 0.08 & -0.25 & 0.18 & 0.12 & 0.51 & 0.29 & 0.10 & 0.06 & 0.72 & -0.12 & 0.49 & 0.34 & 0.06 & 0.32 & 0.07 & 0.41 & 0.02 \\
\hline $\mathrm{R}$ & C24:0 & 0.15 & 0.40 & -0.40 & 0.02 & -0.01 & 0.99 & 0.27 & 0.14 & 0.02 & 0.89 & -0.05 & 0.76 & 0.26 & 0.15 & 0.24 & 0.18 & 0.32 & 0.07 \\
\hline
\end{tabular}

elation between ceramides and inflammatory cytokines. Data are representative of samples collected at wk 0 and 8 relative to the start of intervention.

${ }^{2} \mathrm{MCP}$, monocyte chemoattractant protein.

${ }^{3} \mathrm{MDC}$, macrophage-derived chemokine.

${ }^{4}$ TARC, thymus- and activation-regulated chemokine. 

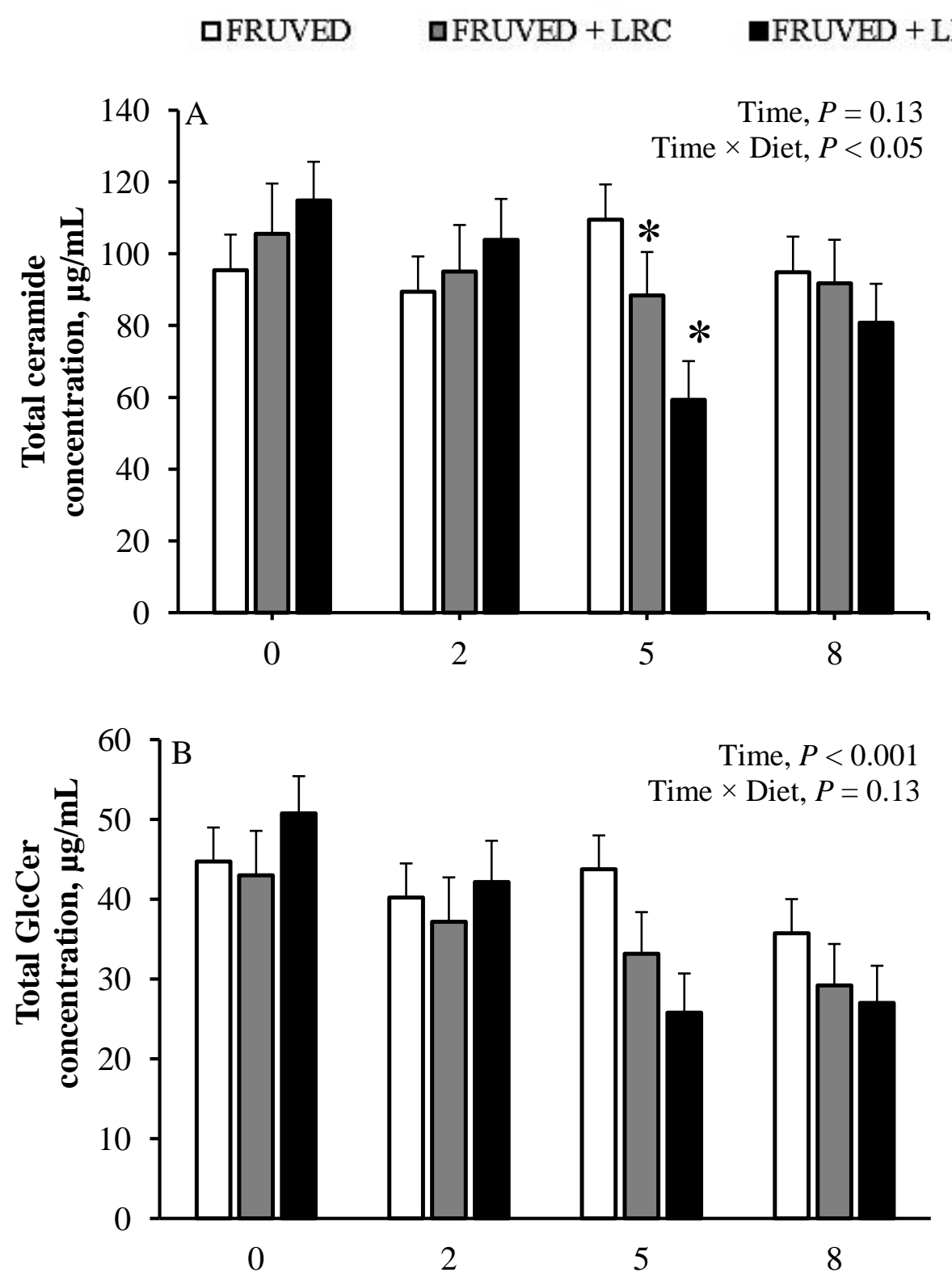


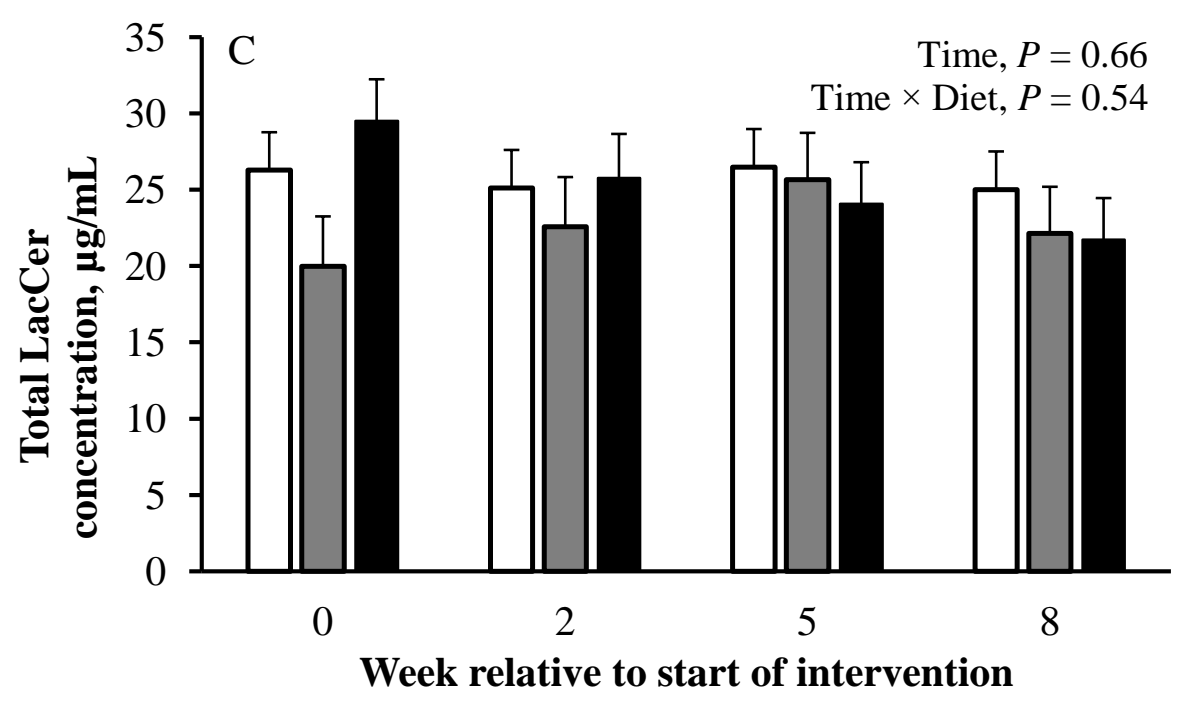

Figure 1 

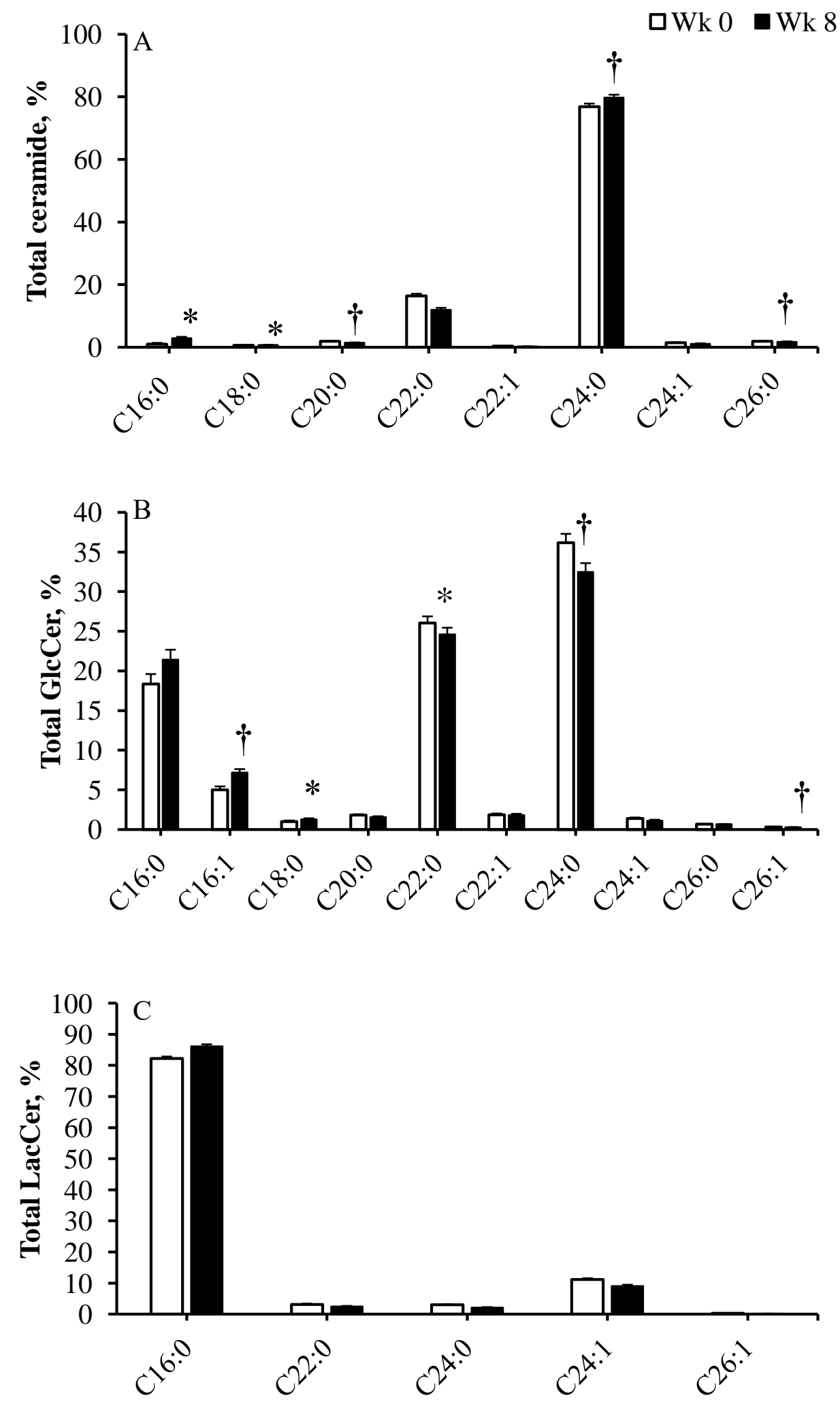

Figure 2. 


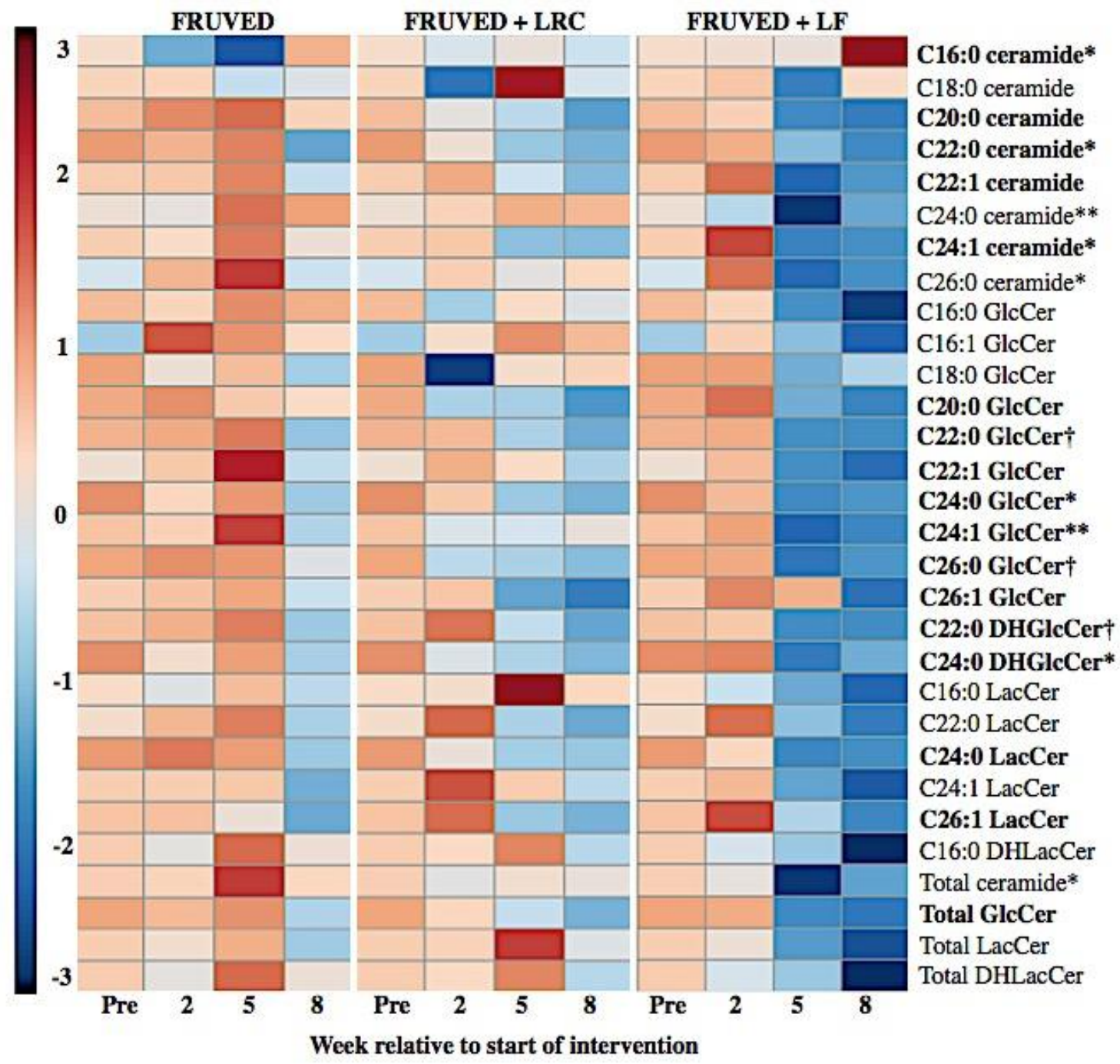

Figure 3. 

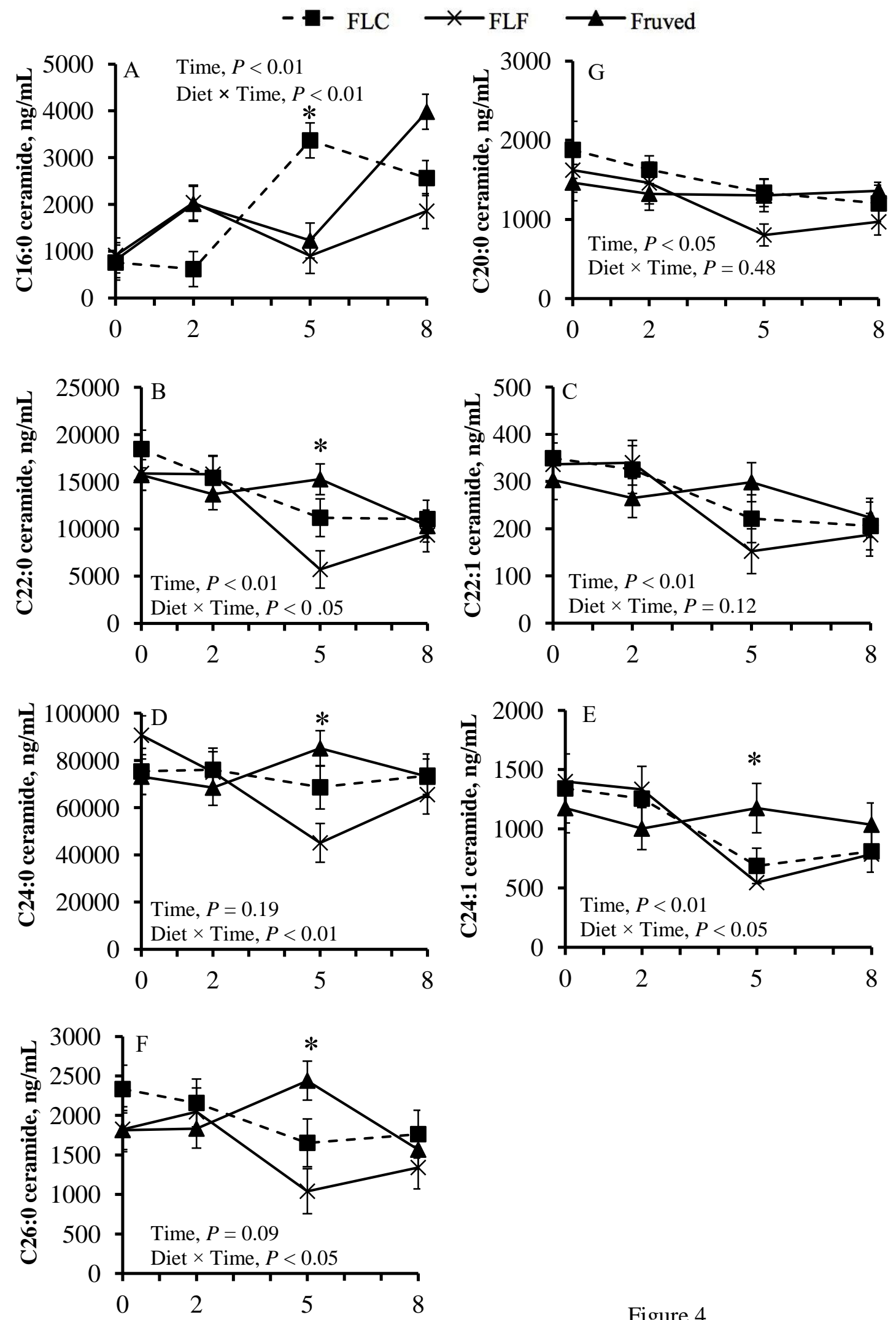

Figure 4.

Week relative to start of intervention 


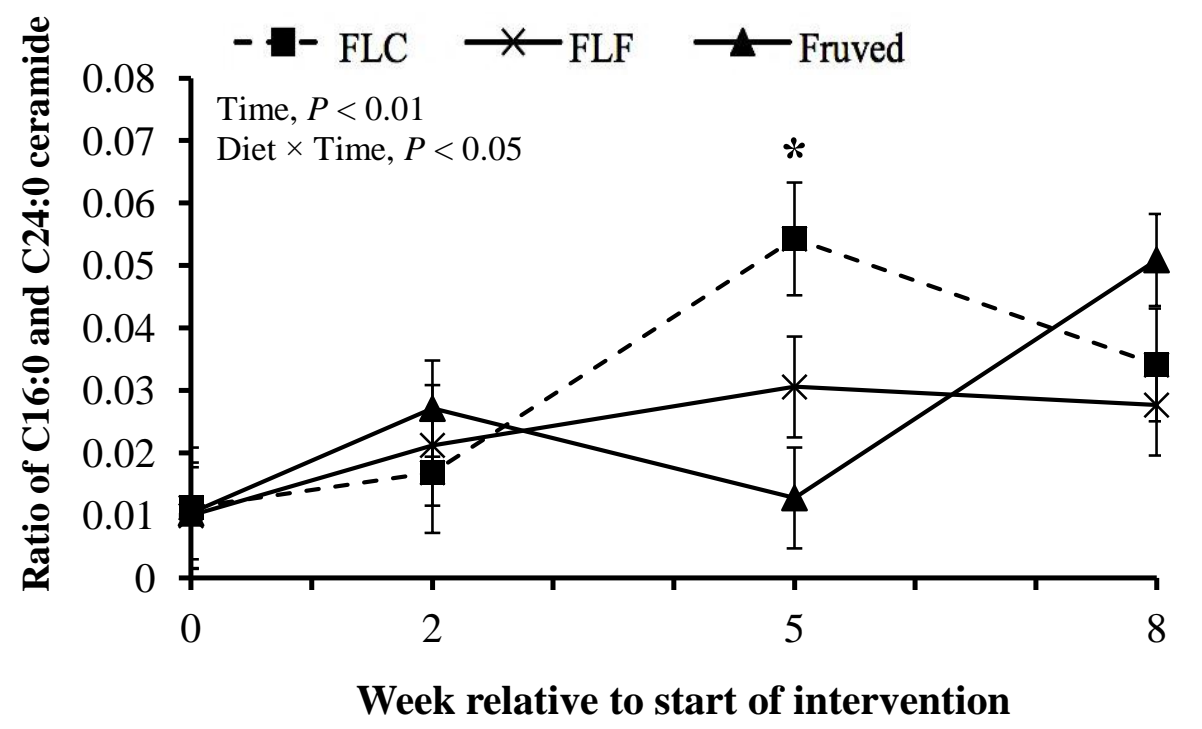

Figure 5. 

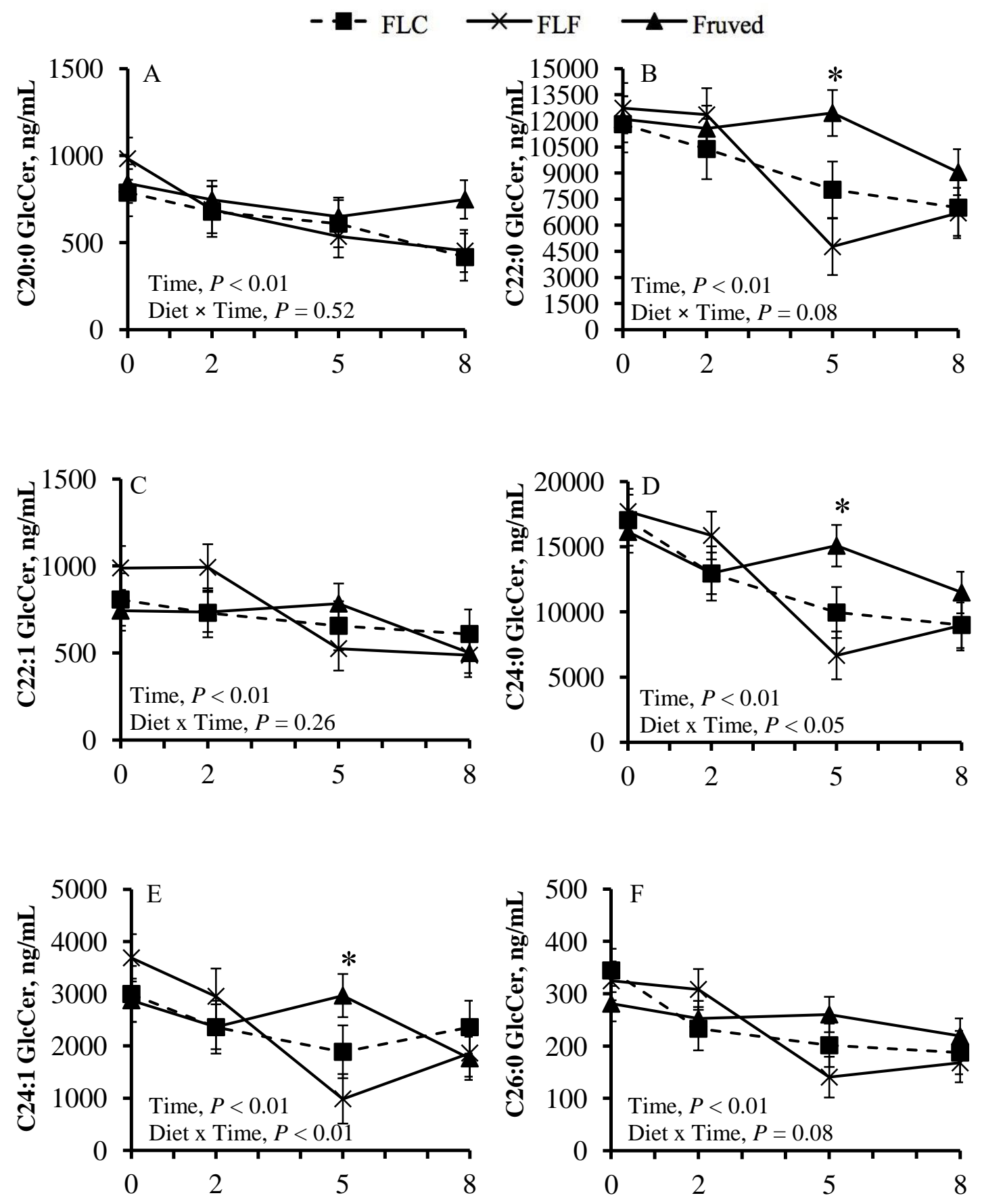


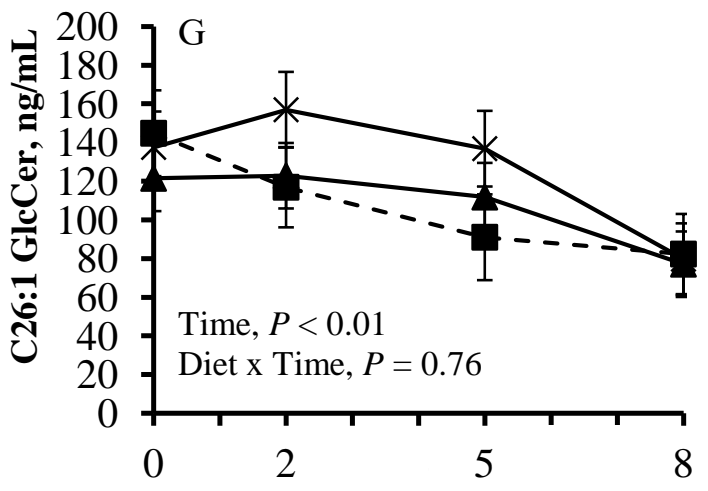

Figure 6.

Week relative to start of intervention 


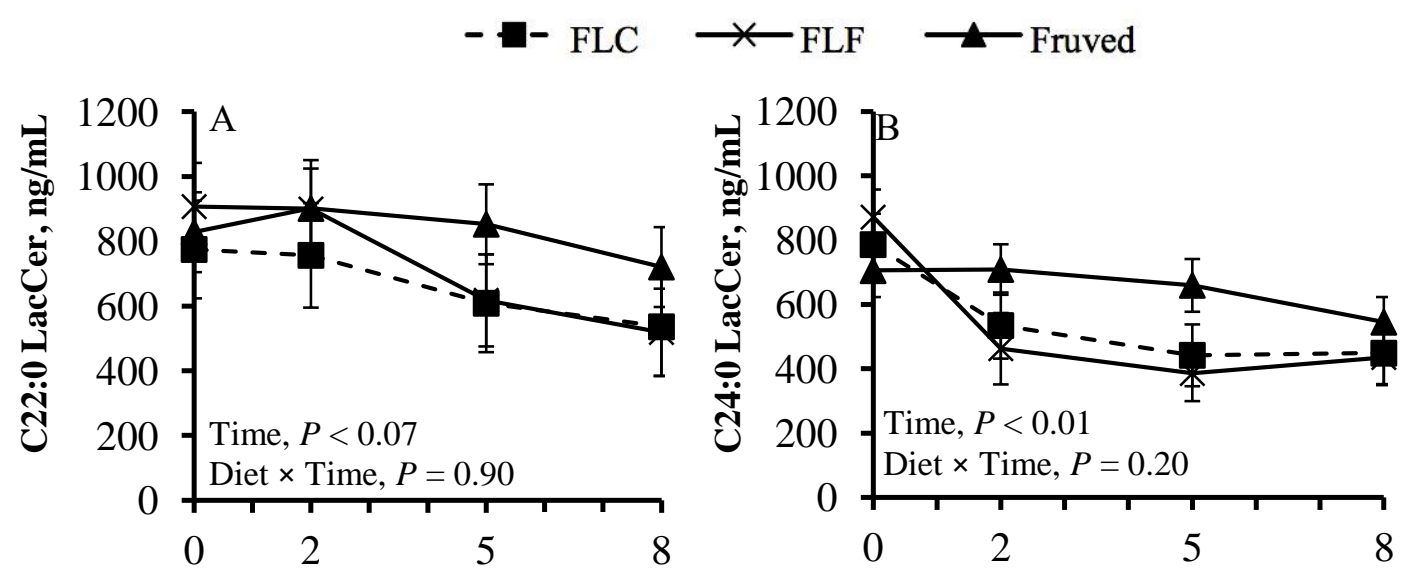

Week relative to start of intervention

Figure 7. 

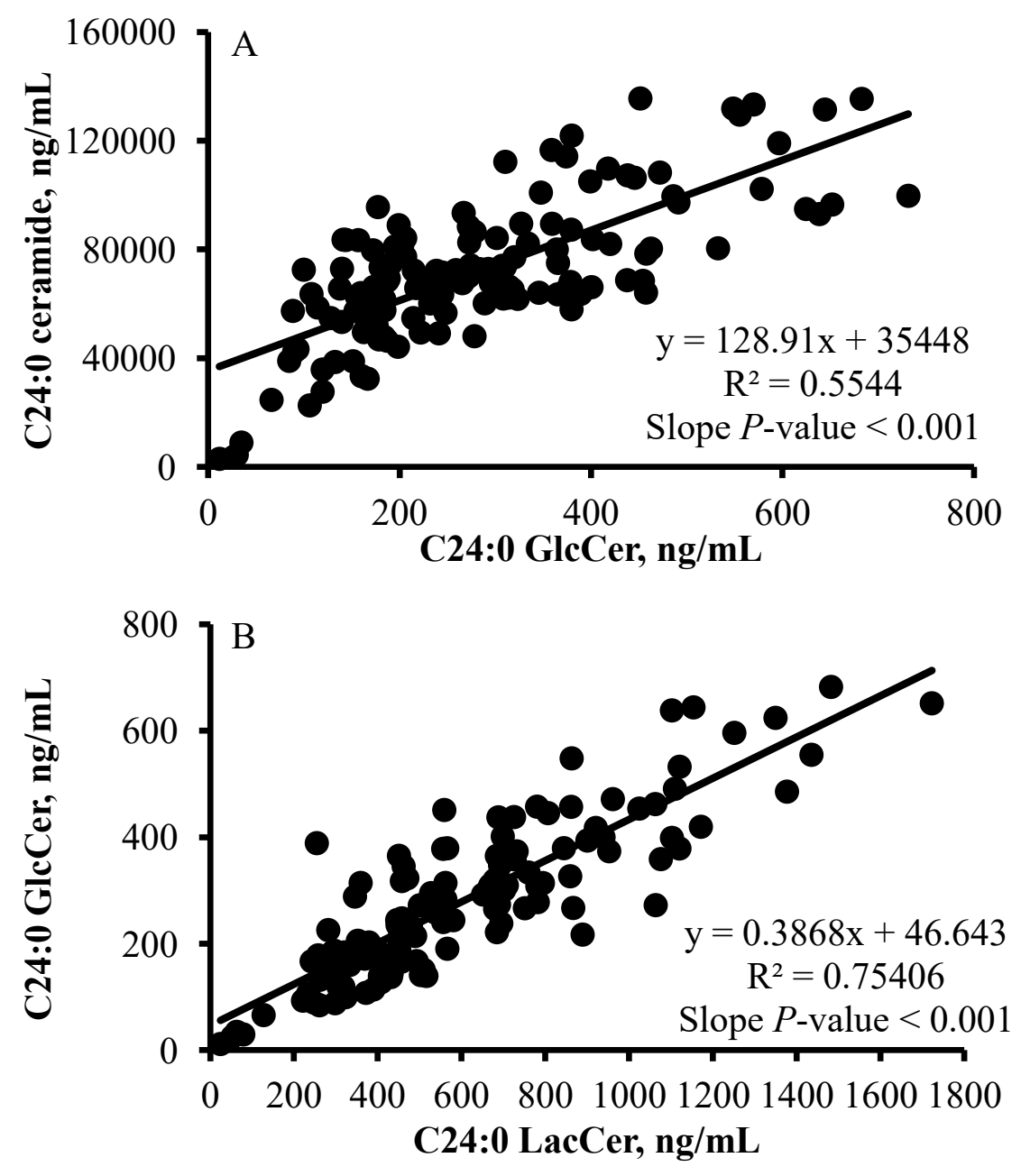

Figure 8. 


\section{Figure Legends}

Figure 1. Total (A) ceramide, (B) GlcCer, and (C) LacCer concentrations in FRUVED (50\% fruit and vegetable), FRUVED + LRC (FRUVED plus low refined carbohydrate), and FRUVED + LF (FRUVED plus low fat) groups throughout intervention. Data are represented as least squares means and their standard errors. Statistical significance compared to FRUVED group within time point. *, $P<0.05$.

Figure 2. Abundance profile of (A) Ceramide, (B) GlcCer, and (C) LacCer in all subjects. Dihydro species are not included. Data are represented as least squares means and their standard errors. Statistical significance compared to pre-intervention (wk 0) measurements within acyl moiety. ${ }^{*}, P<0.05 ; \uparrow, P<0.10$.

Figure 3. Heat map representation of ceramide and glycosylated ceramide changes with intervention. Fruit and vegetable interventions (FRUVED) with or without low intake of refined carbohydrates (LRC) and fat (LF) are shown. Heat map represents the magnitude of meancentered, fold change increases (red) or decreases (blue) for each sphingolipid relative to samples pre-intervention (wk 0). Comparisons should be made within metabolite and intervention. Heat map was generated using MetaboAnalyst 3.0 (Xia et al., 2012). Intervention $\times$ time effects are presented as $* * P<0.01, * P<0.05$, and $\dagger P<0.10$. Significant time effects $(P<0.05)$ are presented in bold.

Figure 4. Changes in circulating ceramides during intervention. Interventions include FRUVED (50\% fruit and vegetable), FRUVED + LRC (FRUVED plus low refined carbohydrate), and FRUVED + LF (FRUVED plus low fat). Statistical significance compared to FRUVED group within time point. Statistical significance compared to FRUVED group within time point. ${ }^{*}, P<$ 0.05 .

Figure 5. Ratio of C16:0 to C24:0 ceramide in serum. Interventions include FRUVED (50\% fruit and vegetable), FRUVED + LRC (FRUVED plus low refined carbohydrate), and FRUVED + LF (FRUVED plus low fat). Statistical significance compared to FRUVED group within time point. Statistical significance compared to FRUVED group within time point. ${ }^{*}, P<0.05$.

Figure 6. Changes in circulating monohexosylceramides (GlcCer) during intervention. Interventions include FRUVED (50\% fruit and vegetable), FRUVED + LRC (FRUVED plus low refined carbohydrate), and FRUVED + LF (FRUVED plus low fat). Statistical significance compared to FRUVED group within time point. *, $P<0.05$.

Figure 7. Changes in circulating lactosylceramides (LacCer) during intervention. Interventions include FRUVED (50\% fruit and vegetable), FRUVED + LRC (FRUVED plus low refined carbohydrate), and FRUVED + LF (FRUVED plus low-fat) compared to FRUVED group.

Figure 8. Random regression analysis between serum concentrations of (A) C24:0 ceramide and monohexosylceramide (GlcCer), and (B) C24:0 GlcCer and lactosylceramide (LacCer). Coefficients of determination $\left(\mathrm{R}^{2}\right)$ represent the association between observed and model predicted values. Data represent measurements for wk $0,2,5$, and 8 relative to the start of interventions. 
Risk Score Abstract

\section{Development of an At Risk for Metabolic Syndrome Score Using Multiple Measures}

Oluremi A. Famodu, MS, RDN, Ida Holásková PhD, Pamela J Murray, MD, Melissa D. Olfert, DrPH, RDN

West Virginia University, Morgantown, West Virginia

Background: MetS affects more than $20 \%$ of U.S. adults, where it has been reported that at least $27 \%$ of college students have one component of MetS (i.e. abnormal waist circumference, dyslipidemia, elevated blood pressure, insulin resistance). Because MetS increases an individual's risk of cardiovascular disease, type 2 diabetes, and cancer, epidemiological research requires a tool that accurately assesses one's risk of developing this condition. However, an instrument that assess risk of MetS is limited and weigh only the five components.

Objective: The objective was to develop a tool that evaluates risk of the MetS in college-age students using other individual factors in addition to the common five components of MetS.

Methods: Nutrition and exercises professionals in addition to a pediatric physician met and created the tool in September 2014. In addition to the five components, the tool considered nutritional status, physical inactivity, ethnicity, family history and current medication. MetS risk was identified in thirty-seven young adults at West Virginia University (WVU) from the FRUVEDomic Pilot Study (Clinical Trial Record 1409433435). Using the WVU's School of Public Health MetS Severity Score (Gurka et al 2014) and the American Heart Association Score (AHA My Life Check® 2016) correlations were completed between our score and other common tools.

Results: Pearson's correlation showed a significant association $(r=0.49$ CI $(0.19,0.70)$ $\mathrm{p}=0.0024$ ) between WVU's Public Health Z-score with our tool's score. In contrast, a correlation was seen with the AHA Score and our tool $(r=-0.35$, CI $(-0.60,-0.04) p=0.03)$.

Conclusion: Our instrument matches up to the most validated tools that currently assess MetS severity, however our tool takes into account variables that highly contribute to risk of developing MetS and other disease. Future analysis will be to validate our tool with a larger sample size. Development of this tool will help improve primary intervention strategies on individuals at risk of disease. 
Metabolic Syndrome Cohort Abstract

\title{
Fruit and Vegetable Diet Intervention in Young Adults with Metabolic Syndrome: Fruvedomics Pilot Study
}

\author{
RL Clark, OA Famodu, ML Barr, PJ Murray, MT Downes, P Chantler, JW McFadden, CF Cuff, \\ IM Olfert, SE Colby, MD Olfert \\ West Virginia University, Davis College of Human Nutrition \& Food, and School of Medicine, \\ Morgantown, WV
}

Objective: Design and implement a multi-disciplinary primary dietary intervention following the USDA's 2015 Dietary Guidelines for Americans in young adults with metabolic syndrome (MetS).

Methods: University students (18-30 years old) with MetS were recruited via flyers, class announcements, and a phone screening questionnaire. Screened individuals completed anthropometric measures (height, weight, body fat percentage) and fasting blood lipids and serum glucose levels. Seventeen of forty-one of the initially screened participants were diagnosed with MetS. Participants $(\mathrm{n}=17)$ were assessed with: arterial stiffness, complete blood lipid panel, anthropometric measures, 24-hour diet recall, and body composition measurements (Bod Pod). Participants had an 8-week diet intervention with weekly consultations reviewing data from food logs, food receipts, and adherence to the diet. Participants were given a culinary toolkit at the beginning of the intervention and had the opportunity to attend two group food demonstrations throughout the 8 weeks.

Results: Study participants $(n=17$, male $=6$; females $=11)$ consisted of the following demographics. Ethnicity was 76\% white, $17 \%$ African American, and 5\% Asian. Mean age was $22 \pm 3.43$ years old. Fruit and vegetable intake increased from pre-assessment to 8 weeks. Mean fruit intake of the group increased from 0.6 cups to 1.9 cups per day. Mean vegetable intake of the group increased from 0.97 cups to 2.54 cups per day. A repeated measures analysis of both fruit $(\mathrm{p}<0.0001)$ and vegetable $(\mathrm{p}<0.0008)$ intake from pre-assessment to week 8 was found to be significant. CRP and cholesterol were higher than clinical normal values $(11.59 \pm 11.29 \mathrm{mg} / \mathrm{dl}$ and $171.39 \pm 27.45 \mathrm{mg} / \mathrm{dl}$, respectively). MetS risk score was assessed using the West Virginia University MetS severity calculator. All participants were at a high risk with scores $\geq 6$. Using the NCEP Panel III Risk Scoring for MetS- the following is the percentage of the participants $(\mathrm{n}=17)$ who met the qualification for that category prior to the invention: Waist Circumference $(100 \%)$, blood pressure $(23.5 \%)$, fasting triglycerides $(47 \%)$, high density lipoprotein (82\%), and fasting blood glucose $(70 \%)$.

Conclusions and Implications: Implementation of a free-living 8-week diet with intensive education and accountability gave participants the knowledge, skills, and feedback to improve fruit and vegetable consumption. Identification of young adults with MetS allows for implementation of an intervention to improve diet and decrease the individual's risk factors for cardiovascular diseases and diabetes. 


\section{Modified Sleep Tool Paper}

\section{Abstract \\ Shortening of the Pittsburgh Sleep Quality Index Survey using Factor Analysis}
OA Famodu MS, RDN ${ }^{1}$, ML Barr BS ${ }^{1}$, I Holásková $\mathrm{PhD}^{1}$, W Zhou $\mathrm{PhD}^{2}$, JS Morrell $\mathrm{PhD}^{3}$, SE Colby $\mathrm{PhD}^{2}, \mathrm{RDN}, \mathrm{MD}$ Olfert DrPH, MS, RDN ${ }^{1}$
West Virginia University ${ }^{1}$, University of Tennessee ${ }^{2}$, University of New Hampshire ${ }^{3}$

Background: Lengthy surveys have the potential to burden participants and cause inaccuracies if participants rush or omit information. Conducting analyses to shorten existing validated surveys are beneficial.

Objective: Shorten the Pittsburgh Quality Sleep Index (PSQI) that assesses sleep habits and distinguishes healthy sleepers from clinically problematic sleepers.

Participants and Methods: A confirmatory factor analysis (FA) was conducted on a large sample ( $\mathrm{n}=1246)$ of college students to check the reliability of the shortened survey after dropping select items. Nonparametric correlation analyses (Spearman's rho) was conducted between the cumulative sleep scores of the shortened and original surveys and agreements tests (Kappa and McNemar's Test) were used to measure the agreement of the surveys in classifying good and problematic sleepers.

Results: Eight factors were examined using promax rotation to allow for correlated variables and $74.0 \%$ of variability was explained. The FA resulted in 12 selected questions ("shortPSQI"), which corresponded to 6 of the 7 components of the original survey (sleep duration, sleep latency, sleep efficiency, sleep disturbances, sleep medications, and daytime dysfunction). High correlation was found between the composite score of the original survey and the "shortPSQI" $(\mathrm{rho}=0.93, \mathrm{p}<0.001)$. When the composite score was converted to the categorical variable of poor or bad sleepers, the agreement test indicated strong agreement (Kappa 0.83, $\mathrm{p}<0.001$ ) with the original survey results.

Conclusion: The validated, 19-item PSQI survey was shortened to 12 items and tests of correlation and agreement indicate that the "shortPSQI" may be an acceptable alternative to the original survey. 


\section{Introduction}

Research conducted in higher education institutions often rely on survey data to gather indicators of health (including on behavior, knowledge, and other health-related factors). However, response burden is one potential issue that limits the amount of data collected from survey distribution. Factors associated with response burden are questionnaire length, cognitive ability to complete the survey, and type of questionnaire interface [230]. In particular, lengthy surveys result in fewer respondents starting or completing questionnaires [231] and differences in processing questions near the end, leading to omitted information [232] and/or uniform answers [233]. Given the need to maintain high quality research, investigators have begun to explore ways to decrease response burden. Prolonged survey length has been used as a rationale to reduce the number of items in existing questionnaires [234]. Some studies take a smaller subset of questions from validated surveys with the intention of reducing response burden [235]. However, these truncated surveys need to be re-validated before used in research.

Quantitative and qualitative attributes of sleep are often investigated to assess the relationship between sleep and overall health. College students are increasingly studied in the field of sleep due to their abnormal schedules and decreased sleep quality [236]. College students' unhealthy lifestyle modifications could be due to social and academic demands that these young individuals are often facing [236]. The adoption of unhealthy lifestyles impacts not only sleep, but also diet [237], physical activity [238], and mental health [239]. Often these global health-related behaviors are associated with decreased capacity to succeed in school [239]. Although there are "gold standard" tools, such as polysomnography, to analyze sleep quality [240], these tools tend to be expensive and may not be feasible for use with larger sample sizes. That being so, most investigators use published survey scales related to sleep quantity and 
quality. The Pittsburgh Sleep Quality Index (PSQI) [115] is a 19-item, self rating tool that assesses sleep quality and discriminates between "good" and "poor sleepers". The PSQI has been shown to have a high degree of internal consistency (Cronbach's alpha 0.83 ) and has been validated against clinical and laboratory diagnoses of "good" and "poor" sleepers following 18 months of field-testing [115]. A commonly used research tool like the PSQI that has been validated against objective measures (can be further examined to see whether shortening maintains adequate statistical validity with the original tool. This in turn would reduce participant response burden associated with using this sleep assessment tool.

Therefore, the present study used a factor analysis to shorten the 19-item, validated PSQI survey using a large sample of college students and compared the calculated variables of the shortened version to the original version.

\section{Methods}

\section{Sample}

College freshman across eight universities (West Virginia University, University of Tennessee, University of Maine, Syracuse University, University of Florida, Kansas State University, University of Alabama and South Dakota State University) were recruited in the fall of 2015 to participate in the Get Fruved study. Get Fruved is a USDA-funded project designed to prevent the development of overweight and obesity among older adolescents by developing a peer-led, interactive, social marketing intervention focusing on dietary quality, increased physical activity, and stress management along with environmental support. All incoming first year students were invited to participate. To be eligible to participate, the students had to be consuming less than the recommended intakes for fruits or vegetables and meet one additional criteria (including first generation college student, racial/ethnic minority, be overweight or obese, 
have a parent who is overweight or obese, or come from a lower affluent household). A total of 1374 college students were interested and eligible to be in the study, however 1246 students completed the PSQI survey in its entirety. Each university's Institutional Review Board approved the study and every participant signed a consent form. Participants then completed a behavioral survey that included questions on many different health-related areas including fruit and vegetable intake (NCI Fruit and Vegetable Screener) [241], stress (Cohen's Perceived Stress Scale) [116], sleep behavior (PSQI) [115], physical activity (International Physical Activity Questionnaire) [242] and other health measurements.

\section{Original PSQI Measuring Instrument}

In the sleep section of the survey, students were asked to recall their sleep habits over the past month and answer 19 individual questions, related to seven main components: sleep quality, sleep latency, sleep duration, habitual sleep efficiency, sleep disturbance, use of sleep medication, and daytime dysfunction. PSQI is described in more detail by Buysse et al. [115]. Table 1 shows the 19 items of the original scale and the seven components to which each item belonged. Following the protocols established by Buysse et al. (1989), each component received a score based on a Likert scale of $0-3$, where a score of 0 reflected the positive extreme ("Not during the past month") and score of 3 reflected a negative extreme. A global PSQI score was generated for each participant after summing the seven components (all weighted equally on a 0 3 scale), with a final range of 0-21 where higher scores indicated worse sleep quality. A global PSQI score > 5 is indicative of poor sleep quality, relative to clinical and laboratory measures. 
Statistical Test for Developing "shortPSQI"

In order to shorten the original 19-item PSQI survey, a confirmatory factor analysis (FA) with promax rotation was conducted on all 19 questions. A 0.60 or higher on the loading plot was used as a cut-off to determine the factors kept in the "shortPSQI".

Statistical Test for Testing Validity of "shortPSQI"

For validation of the FA, a nonparametric correlation (Spearman's rho) was used to measure the consistency between the global PSQI sleep score of the "shortPSQI" with the PSQI of the original survey. In addition, Kappa and McNemar's tests were used to measure the agreement of the survey in detecting number of "good" and "bad" sleepers. Efficacy tests of specificity and sensitivity were also conducted to measures the proportion of "poor" and "good" sleepers detected by the shortened survey to the original questionnaire. A Receiver Operating Characteristic (ROC) curve was constructed to ensure the most acceptable degree of sensitivity and specificity were maintained in order to validate the PSQI score cut-off that distinguished between "poor" and "good" sleepers in the "shortPSQI".

All statistical analyses were completed using JMP and SAS software (JMP®, Version

Pro 11, SAS Institute Inc., Cary, NC, Copyright @2013; SAS®, Version 9.3, SAS Institute Inc., Cary, NC, Copyright (O2002-2010). Significance criterion alpha for all tests was 0.05 .

\section{Results}

Developing “shortPSQI” Survey

Utilizing the promax rotation method, eight underlying latent factors were identified (likelihood ratio tests; no common factors $\mathrm{p}<0.001$ and sufficiency of 8 factors $\mathrm{p}=0.30$ ), explaining a total of 74.0 cumulative percent of variability. The eigenvectors on each factor 
identified one or two questions that had a very high coefficient (minimum of 0.60 ), meaning a high contribution of these questions to variability for each factor. Using such criteria, 12questions were selected for the "shortPSQI" and corresponded to 6 of the 7 components of the original survey (sleep duration, sleep latency, sleep efficiency, sleep disturbances, using sleep medications, and daytime dysfunction) (Table 1). The omitted questions of all loadings less than 0.60 belonged to other sleep disturbances (Questions 7, 8, 10,11, and 12) and overall sleep quality (Question 19).

Scoring of "shortPSQI"

Components that had all questions with a loading of 0.60 or higher were first organized and evaluated following the original method developed by Buysse et al. (1989). These components included sleep duration, sleep latency, sleep efficiency, and use of sleep medications. If questions were omitted in the loading plot and contributed to certain component score (i.e. sleep disturbances and daytime function), the summation of all questions attained a smaller maximum as in the original. These final scores for the whole component were proportioned to fit a range of 0 to 3 . To illustrate the algorithm, the "sleep disturbance" component in the original survey contained nine questions. Each of them were first scored on a 0-3 Likert scale, based on the answers, and then added together to get a total score between 0-27 for this component [115]. In order for this sum to be on the final scale of $0-3$, the total score was divided into four corresponding subsets; zero, and the remaining into 3 equal-distance portions; $\geq 1$ and $\leq 9$ (given a score of 1), $>9$ and $\leq 18$ (given a score of 2), and $>18$ (given a score of 3), proportional to the max value 27 . Having a zero in this component meant the individual had none of these disturbances in the past month, and three (>18) indicating the individual experienced a high amount of disturbances over the past month. The same method was applied to the 
"shortPSQI" where we first scored our four remaining questions on a 0-3 scale, then summed the questions scores to generate a total between 0-12. By dividing this into three equal subsets and a zero category, we derived to $0, \geq 1$ and $\leq 4$ corresponding to $1,>4$ and $\leq 8$ corresponding to 2 , and $>8$ corresponding to final component score of 3 (See Appendix Scoring "shortPSQI").

The daytime dysfunction component of the original survey had two questions. Based on the FA analysis, one question was eliminated, so the score included only the remaining question. Thus, this single score became the total representing the daytime dysfunction component.

Lastly, because the question of overall sleep quality was not included in the loading plot, the sleep quality component was dropped from the "shortPSQI", therefore there were 6 components that remained. If all 6 components scored between 0 and 3 points, this would result in a global PSQI ranging between 0 and 18 , instead of the original 0 and 21 . In order to come up with the categorical variable of "good" and "poor" sleepers, a cross multiplication approach was used with the cut-off threshold of 5 from the original scale of 0 to 21 [115]. Using this method, a global PSQI score $>4$ on the "shortPSQI" was indicative of a poor quality sleeper.

\section{Validity testing of "shortPSQI"}

The average global score of the original survey was 5.23 (SD 2.54) in 1246 college students who completed the entire survey (Table 2). The "shortPSQI" averaged 3.98 (SD 2.22) using the same data. Correlations between the original survey and the "shortPSQI" is depicted in Figure 1. The "shortPSQI" showed a significant correlation with the original global scale $(\rho=$ $0.93, \mathrm{p}<0.001)$.

After the "good" and "bad" sleepers were calculated in each of the surveys, Kappa showed significant strong agreement with the "shortPSQI" and original survey (Kappa 0.83, p 
$<0.001)$ (Table 3). However, the test statistics also showed significance $(\mathrm{p}<0.001)$ in the McNemar's test which means lack of agreement. When looking at sensitivity of the "shortPSQI" in detecting the proportion of "poor" sleepers of those identified as "poor" sleepers by the original, the "shortPSQI" correctly identified 84\% (424/507). Specificity (the proportion of "good" sleepers detected by the shortened method of the original "good" sleepers) was also high, where the "shortPSQI" correctly identified 98\% (721/739).

Figure 2 presents the ROC curve based off 5 possible cut-off PSQI scores in determining "poor" versus "good" sleepers for the "shortPSQI" and their corresponding sensitivities and specificities (Table 4). The cut-off 4, which we derived mathematically (See Scoring of "shortPSQI"), demonstrates the highest sensitivity and specificity compared to the other cut-off points.

\section{Discussion}

Lengthy surveys are likely to overburden participants and increase inaccuracies if participants rush or omit information to finish. Shortening existing validated surveys may help limit time it takes for participants to complete these surveys and improve data collection efforts. The results of this study are encouraging for downsizing the 19-item PQSI survey to assess sleep habits and sleep quality. Questionnaire length was reduced by over one third with minimal loss in original survey components. The 12-item "shortPSQI" reached similar conclusions based upon the global score and categorical variable compared to the original survey. Thus, the shortened version of the questionnaire represents a plausible alternative to the original PSQI.

While scoring the "shortPSQI", a concern arose due to a latent factor that excluded more than $50 \%$ of the questions about individual sleep disturbances. This could be the reason why we observed discordance by McNemar's test because we detected slightly less "poor" sleepers than 
the original survey ( $36 \%$ and $41 \%$ respectively). It is expected when the "shortPSQI" is administered, the individual reasons of various sleep disturbances that were excluded in our FA (i.e. cannot breathe comfortable, being too cold, being too hot, a bathroom break, and having a bad dream) would all be answered in the category "other disturbances". If choosing between the forms, it is important to consider the depth of sleep quality the researcher hopes to achieve from their sample population. The 19-item survey defines more levels of sleep disturbances than does the 12-item survey ("shortPSQI"), but we feel that maintaining of the "other" sleep disturbance question in the shortened version will correct this underestimation. However, the shorter version is attractive for larger population samples with time constraints or those who wish to administer surveys with many items.

In conducting the analyses to shorten the original PSQI survey, there are some methodological questions that arise. First, it should be noted that the analyses used to derive the conclusions of the 12-item PSQI survey were based on the data from the 19-item PSQI distributed to college students. We assume that the same or better results will be obtained when the "shortPSQI" is administered alone. There is also the concern of the FA excluding the "sleep quality" component in the shortened version. Although it was adjusted for in the global PSQI score of the 12-item questionnaire, there is still an issue of whether this component is needed to measure overall quality of sleep in a participant. Lastly, although we feel like this was a large enough data set to be able to decrease the number of items in the original survey, this survey was only distributed to young adults attending college, therefore this may not be generalizable to other samples. Thus, further research is encouraged in other populations.

In conclusion the validated, 19-item PSQI survey was shortened to a 12-item survey ("shortPSQI") using a factor analysis on responses from over one thousand students. Testing 
demonstrated that the "shortPSQI" correlated and agreed with the original survey and could potentially be used to derive the same outcomes with reduced respondent burden. Further validation would include administering the "shortPSQI" and original survey to a new sample. 


\section{Tables/Figures}

Table 1. Items of the 19-Item (original) and 12-item ("shortPSQI") survey

\begin{tabular}{|c|c|c|c|c|c|c|c|c|}
\hline Questions of Original Survey & \multicolumn{7}{|c|}{ Components of Original Survey } & "shortPSQI" Questions \\
\hline & I & II & III & IV & $\mathbf{V}$ & VI & VII & \\
\hline 1. When have you usually gone to bed? & & $\mathrm{X}$ & & & & & & $\mathbf{X}$ \\
\hline 2. How long (in minutes) has it taken you to fall asleep at night? & & & $\mathrm{X}$ & & & & & $\mathbf{X}$ \\
\hline 3. What time have you usually gotten up in the morning? & & $\mathrm{X}$ & & & & & & $\mathbf{X}$ \\
\hline 4. How many hours of actual sleep did you get at night? & $\mathrm{X}$ & $\mathrm{X}$ & & & & & & $\mathbf{X}$ \\
\hline 5. Cannot get to sleep within 30 minutes & & & $\mathrm{X}$ & & & & & $\mathbf{X}$ \\
\hline 6. Wake up in the middle of the night or early morning & & & & $\mathrm{X}$ & & & & $\mathbf{X}$ \\
\hline 7. Have to get up to use the bathroom & & & & $\mathrm{X}$ & & & & \\
\hline 8. Cannot breath comfortable & & & & $\mathrm{X}$ & & & & \\
\hline 9. Cough or snore loudly & & & & $\mathrm{X}$ & & & & $\mathbf{X}$ \\
\hline 10. Feel too cold & & & & $\mathrm{X}$ & & & & \\
\hline 11. Feel too hot & & & & $\mathrm{X}$ & & & & \\
\hline 12. Have bad dreams & & & & $\mathrm{X}$ & & & & \\
\hline 13. Have pain & & & & $\mathrm{X}$ & & & & $\mathbf{X}$ \\
\hline 14. Other reason(s), please describe & & & & $\mathrm{X}$ & & & & $\mathbf{X}$ \\
\hline 15. How often do you have trouble sleeping because of this reason(s) & & & & $\mathrm{X}$ & & & & $\mathbf{X}$ \\
\hline 16. How often have you taken medicine to help you sleep & & & & & $\mathrm{X}$ & & & $\mathbf{X}$ \\
\hline $\begin{array}{l}\text { 17. How often have you had trouble staying awake while driving, } \\
\text { eating meals, or engaging in social activity? }\end{array}$ & & & & & & & $\mathrm{X}$ & \\
\hline $\begin{array}{l}\text { 18. How much of a problem has it been for you to keep up } \\
\text { enthusiasm to get things done? }\end{array}$ & & & & & & & $\mathrm{X}$ & $\mathbf{X}$ \\
\hline 19. How would you rate your overall sleep quality? & & & & & & $\mathrm{X}$ & & \\
\hline
\end{tabular}

Note: The introduction read, "The following questions relate to your usual sleep habits during the past month only. Your answers should indicate the most accurate reply for the majority of day and nights in the past month". Questions 1-4 ask the person to give an open-ended response. Questions 5-15 starts with a statement asking the participant "During the past month, how often have you had trouble sleeping because you..." with answers of "Not during the past month", "Less than once a week", "Once or twice a week", and "Three or more times a week". Questions 16 and 17 are on the same scale, but Q18 is from "No problem at all" to "A very big problem". Lastly, Q19 is a Likert scale of "Very good" to "Very bad".

I = Sleep Duration; II = Sleep Efficiency; 3 = Sleep Latency; IV = Sleep Disturbances; V = Use of Medication; VI = Sleep Quality; VII = Daytime Dysfunction 
Table 2: Global PSQI Score of the Original and the "shortPSQI"

\begin{tabular}{lll}
\hline Survey $(\mathrm{n}=1246)$ & Mean & SD \\
Original 19-item PSQI & 5.23 & 2.54 \\
Short 12-item PSQI & 3.98 & 2.22 \\
\hline
\end{tabular}

Note: SD; standard deviation 
Table 3: Agreements Tests between "Good" and "Bad" Sleeper Scored by Original and "shortPSQI"

\begin{tabular}{|c|c|c|c|}
\hline \multirow{2}{*}{ ORIGINAL SURVEY } & \multicolumn{2}{|c|}{ "shortPSQI" } & TOTAL \\
\hline POOR SLEEPRS & BAD SLEEPERS & GOOD SLEEPERS & \\
\hline GOOD SLEEPERS & 424 & 83 & 507 \\
\hline total & 18 & 721 & 739 \\
\hline
\end{tabular}

Kappa value 0.83 indicates an agreement $(\mathrm{p}<0.001)$ between original and "shortPSQI".

McNemar's Test indicated a lack of agreement $(\mathrm{p}<0.0001)$ between original and "shortPSQI". 
Table 4: Specificity and Sensitivity of Different Cut-Off values for "Good" and "Bad" Sleeper Scored by Original and "shortPSQI"

\begin{tabular}{|c|c|c|}
\hline CUT-OFF VALUE $^{\mathrm{a}}$ (PSQI) & SENSITIVITY $^{\mathrm{b}}$ & SPECIFICITY $^{\mathrm{c}}$ \\
\hline 3 & $497 / 507=98 \%$ & $583 / 739=79 \%$ \\
\hline 4 & $424 / 705=84 \%$ & $721 / 739=98 \%$ \\
\hline 5 & $276 / 507=54 \%$ & $738 / 739=100 \%$ \\
\hline 6 & $167 / 507=33 \%$ & $739 / 739=100 \%$ \\
\hline 7 & $86 / 507=17 \%$ & $739 / 739=100 \%$ \\
\hline
\end{tabular}

${ }^{a}$ PSQI cumulative score greater than this cut-off indicates "poor" sleeper.

betecting the proportion of "poor" sleepers determined by "shortPSQI" out of original survey "poor" sleepers.

"Detecting the proportion of "good" sleepers determined by "shortPSQI" out of original survey "good" sleepers. 
Figure 1:

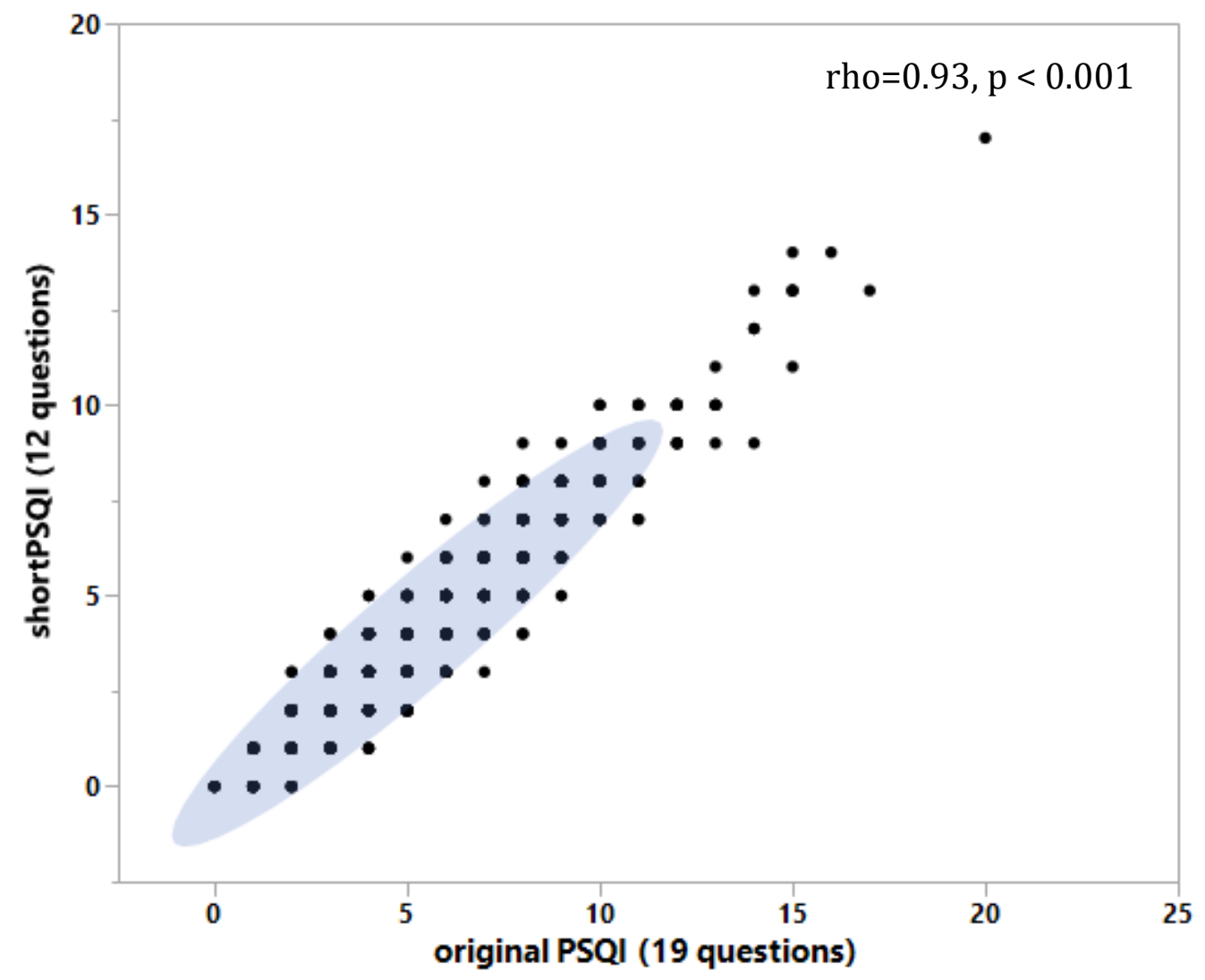


Figure 2:

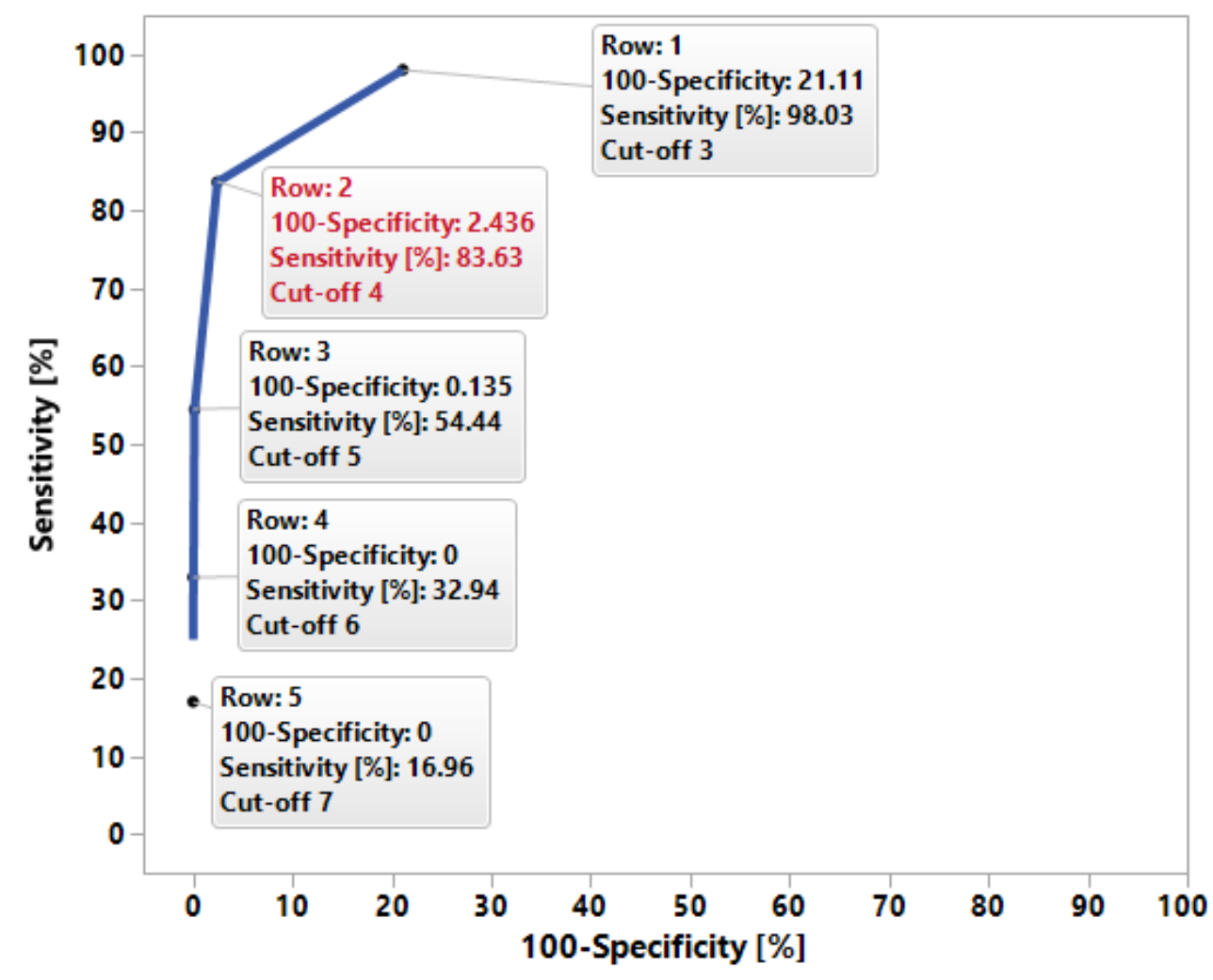


Figure 1: Correlation between Original PSQI and "shortPSQI". A non-parametric correlation analysis detected a significant positive correlation $(\mathrm{rho}=0.93, \mathrm{p}<0.001)$ between the cumulative scores of the "shortPSQI" and the original.

Figure 2: Receiver Operator Characteristic (ROC) Curve. The ROC curve is generated from 5 possible cut-off PSQI scores by determining "poor" versus "good" sleepers for the "shortPSQI" and original survey as gold standard and the corresponding sensitivities and specificities. The highlighted cut-off (PSQI cumulative score $>4$ ) demonstrates the highest sensitivity and specificity (lowest 100-specificity) compared to the other cut-off values. 


\section{Appendix}

\section{Scoring "shortPSQI"}

Name

ID \#

Date Age

Instructions:

The following questions relate to your usual sleep habits during the past month only. Your answers should indicate the most accurate reply for the majority of days and nights in the past month.

Please answer all questions.

1. During the past month, when have you usually gone to bed?

2. During the past month, how long (in minutes) has it taken you to fall asleep each night?

3. During the past month, when have you usually gotten up in the morning?

4. During the past month, how many actual hour of sleep did you get at night? (This may be different than the number of hours you spend in bed.)

For each of the remaining questions, check the one best response. Please answer all questions.

5. During the past month, how often have you had trouble sleeping because you...

a. Cannot get to sleep within 30 minutes

Not during the past month__ Less than once a week__ Once or twice a week_

Three or more times a week

b. Wake up in the middle of the night or early morning

Not during the past month__ Less than once a week__ Once or twice a week_ Three or more times a week

c. Cough or snore loudly

Not during the past month__ Less than once a week__ Once or twice a week__

Three or more times a week

d. Have pain

Not during the past month__ Less than once a week__ Once or twice a week_

Three or more times a week

e. Other reason(s), please

describe

How often during the past month have you had trouble sleeping because of this?

Not during the past month__ Less than once a week__ Once or twice a week Three or more times a week

6. During the past month, how often have you taken medicine (prescribed or "over the counter") to help you sleep? 
Not during the past month__ Less than once a week__ Once or twice a week_ Three or more times a week

7. During the past month, how much of a problem has it been for you to keep up enthusiasm to get things done?

Not during the past month__ Less than once a week__ Once or twice a week_ Three or more times a week

\section{Scoring}
Component 1: Sleep Latency $\quad$ \#2 Score (<15 (0), 16-30min (1), 31-60 min (2), >60min (3))
$\mathrm{C} 1$
Component 2: Sleep Duration
$\mathrm{C} 2$
Component 3: Sleep Efficiency
$\mathrm{C} 3$
(total \# of hours asleep) / (total \# of hours in bed) x 100
$>85 \%=0,75-84 \%=1,65-74 \%=2,<65 \%=3$

Component 4: Sleep Disturbances

C4

Component 5: Sleep Medications

C5

Component 6: Daytime Dysfunction

C6

Add the six component scores together...Global PSQI

A total score of greater than " 4 " is indicative of poor sleep quality
Examine questions $5 \mathrm{~b}$ to $5 \mathrm{e}$ and assign scores for each questions as follows

\section{Response}

Score

Not during the past month

0

Less than once a week

1

Once or twice a week

2

Three or more times a week

3

\# sum of scores $5 b$ to 5 e $(0=0)$

\#6 Score (use the same scoring method as above for sleep disturbances)

\#7 Score (use the same scoring method as above for sleep disturbances) 


\section{References}

1. Lloyd-Jones, D., et al., Heart disease and stroke statistics-2010 update. Circulation, 2010. 121(7): p. e46-e215.

2. $\quad$ Flegal, K.M., et al., Prevalence of obesity and trends in the distribution of body mass index among US adults, 1999-2010. Jama, 2012. 307(5): p. 491-497.

3. Reaven, G.M., The metabolic syndrome: is this diagnosis necessary? The American journal of clinical nutrition, 2006. 83(6): p. 1237-1247.

4. Park, Y.-W., et al., The metabolic syndrome: prevalence and associated risk factor findings in the US population from the Third National Health and Nutrition Examination Survey, 1988-1994. Archives of internal medicine, 2003. 163(4): p. 427-436.

5. Organization, W.H., Global status report on noncommunicable diseases 2010. Geneva: WHO; 2011. 2014.

6. $\quad$ Krauss, R., et al., Revision 2000: a statement for healthcare professionals from the Nutrition Committee of the American Heart Association. The Journal of nutrition, 2001. 131(1): p. 132.

7. US Department of Agriculture, U.D.o.H. and H. Services, Dietary guidelines for Americans, 2010. 2010, US Government Printing Office Washington, DC.

8. Alonso, A., et al., Fruit and vegetable consumption is inversely associated with blood pressure in a Mediterranean population with a high vegetable-fat intake: the Seguimiento Universidad de Navarra (SUN) Study. British Journal of Nutrition, 2004. 92(02): p. 311319.

9. Deshmukh-Taskar, P.R., et al., Dietary patterns associated with metabolic syndrome, sociodemographic and lifestyle factors in young adults: the Bogalusa Heart Study. Public health nutrition, 2009. 12(12): p. 2493-2503.

10. Bazzano, L.A., M.K. Serdula, and S. Liu, Dietary intake of fruits and vegetables and risk of cardiovascular disease. Current atherosclerosis reports, 2003. 5(6): p. 492-499.

11. Ford, E.S., et al., The metabolic syndrome and antioxidant concentrations. Diabetes, 2003. 52(9): p. 2346-2352.

12. Esmaillzadeh, A., et al., Fruit and vegetable intakes, $C$-reactive protein, and the metabolic syndrome. The American journal of clinical nutrition, 2006. 84(6): p. 14891497.

13. Watzl, B., et al., A 4-wk intervention with high intake of carotenoid-rich vegetables and fruit reduces plasma C-reactive protein in healthy, nonsmoking men. The American journal of clinical nutrition, 2005. 82(5): p. 1052-1058.

14. Grosso, G., et al., Mediterranean diet and cardiovascular risk factors: a systematic review. Critical reviews in food science and nutrition, 2014. 54(5): p. 593-610.

15. Willett, W.C., et al., Mediterranean diet pyramid: a cultural model for healthy eating. The American journal of clinical nutrition, 1995. 61(6): p. 1402S-1406S.

16. Velázquez-López, L., et al., Mediterranean-style diet reduces metabolic syndrome components in obese children and adolescents with obesity. BMC pediatrics, 2014. 14(1): p. 175.

17. Esposito, K., et al., Effect of a Mediterranean-style diet on endothelial dysfunction and markers of vascular inflammation in the metabolic syndrome: a randomized trial. Jama, 2004. 292(12): p. 1440-1446. 
18. Mayneris-Perxachs, J., et al., Effects of 1-year intervention with a Mediterranean diet on plasma fatty acid composition and metabolic syndrome in a population at high cardiovascular risk. PLoS One, 2014. 9(3): p. e85202.

19. Vollmer, W.M., F.M. Sacks, and L.P. Svetkey, New insights into the effects on blood pressure of diets low in salt and high in fruits and vegetables and low-fat dairy products. Trials, 2001. 2(2): p. 71.

20. Azadbakht, L., et al., Beneficial effects of a Dietary Approaches to Stop Hypertension eating plan on features of the metabolic syndrome. Diabetes care, 2005. 28(12): p. 28232831.

21. Ebbeling, C.B., et al., Effects of a low-glycemic load vs low-fat diet in obese young adults: a randomized trial. Jama, 2007. 297(19): p. 2092-2102.

22. Pi-Sunyer, F.X., et al., Clinical guidelines on the identification, evaluation, and treatment of overweight and obesity in adults. American Journal of Clinical Nutrition, 1998. 68(4): p. 899-917.

23. Krebs-Smith, S.M., et al., Americans do not meet federal dietary recommendations. The Journal of nutrition, 2010. 140(10): p. 1832-1838.

24. McCracken, M., R. Jiles, and H.M. Blanck, Health behaviors of the young adult US population: behavioral risk factor surveillance system, 2003. Prev Chronic Dis, 2007. 4(2): p. A25.

25. Gordon-Larsen, P., et al., Five-year obesity incidence in the transition period between adolescence and adulthood: the National Longitudinal Study of Adolescent Health. The American journal of clinical nutrition, 2004. 80(3): p. 569-575.

26. Racette, S.B., et al., Weight changes, exercise, and dietary patterns during freshman and sophomore years of college. Journal of American college health, 2005. 53(6): p. 245-251.

27. Lowry, R., et al., Physical activity, food choice, and weight management goals and practices among US college students. American journal of preventive medicine, 2000. 18(1): p. 18-27.

28. Strong, K.A., et al., Weight gain prevention: identifying theory-based targets for health behavior change in young adults. Journal of the American Dietetic Association, 2008. 108(10): p. 1708-1715. e3.

29. Driskell, J.A., M.C. Schake, and H.A. Detter, Using nutrition labeling as a potential tool for changing eating habits of university dining hall patrons. Journal of the American Dietetic Association, 2008. 108(12): p. 2071-2076.

30. Louis, W., et al., Pizza and pop and the student identity: The role of referent group norms in healthy and unhealthy eating. The Journal of social psychology, 2007. 147(1): p. 5774.

31. Poddar, K.H., et al., Low-fat dairy intake and body weight and composition changes in college students. Journal of the American Dietetic Association, 2009. 109(8): p. 14331438.

32. Contento, I., et al., The effectiveness of nutrition education and implications for nutrition education policy, programs, and research: a review of research. Journal of nutrition education (USA), 1995.

33. Ammerman, A.S., et al., The efficacy of behavioral interventions to modify dietary fat and fruit and vegetable intake: a review of the evidence. Preventive medicine, 2002. 35(1): p. 25-41. 
34. Adamson, A.J. and J.C. Mathers, Effecting dietary change. Proceedings of the Nutrition Society, 2004. 63(04): p. 537-547.

35. Kerr, D.A., et al., Connecting Health and Technology (CHAT): protocol of a randomized controlled trial to improve nutrition behaviours using mobile devices and tailored text messaging in young adults. BMC public health, 2012. 12(1): p. 477.

36. Kreuter, M.W. and C.S. Skinner, Tailoring: what's in a name? 2000, Oxford Univ Press.

37. Brug, J., et al., The impact of a computer-tailored nutrition intervention. Preventive medicine, 1996. 25(3): p. 236-242.

38. Wright, J.L., et al., Tailored, iterative, printed dietary feedback is as effective as group education in improving dietary behaviours: results from a randomised control trial in middle-aged adults with cardiovascular risk factors. International Journal of Behavioral Nutrition and Physical Activity, 2011. 8(1): p. 43.

39. Kroeze, W., A. Werkman, and J. Brug, A systematic review of randomized trials on the effectiveness of computer-tailored education on physical activity and dietary behaviors. Annals of behavioral medicine, 2006. 31(3): p. 205-223.

40. Kelly, N.R., S.E. Mazzeo, and M.K. Bean, Systematic review of dietary interventions with college students: directions for future research and practice. Journal of nutrition education and behavior, 2013. 45(4): p. 304-313.

41. Richards, A., K.K. Kattelmann, and C. Ren, Motivating 18-to 24-year-olds to increase their fruit and vegetable consumption. Journal of the American Dietetic Association, 2006. 106(9): p. 1405-1411.

42. Clifford, D., et al., Good Grubbin': impact of a TV cooking show for college students living off campus. Journal of nutrition education and behavior, 2009. 41(3): p. 194-200.

43. Schnoll, R. and B.J. Zimmerman, Self-regulation training enhances dietary self-efficacy and dietary fiber consumption. Journal of the American Dietetic Association, 2001. 101(9): p. 1006-1011.

44. Bandura, A. and R.H. Walters, Social learning theory. 1977.

45. Cox, V., University Students' Eating Behaviours: Implications for the Social Cognitive Theory. 2016, Mount Saint Vincent University.

46. Franko, D.L., et al., Motivation, self-efficacy, physical activity and nutrition in college students: randomized controlled trial of an internet-based education program. Preventive medicine, 2008. 47(4): p. 369-377.

47. Savage, D.C., Microbial ecology of the gastrointestinal tract. Annual Reviews in Microbiology, 1977. 31(1): p. 107-133.

48. Heinken, A. and I. Thiele, Systematic prediction of health-relevant human-microbial cometabolism through a computational framework. Gut Microbes, 2015. 6(2): p. 120-130.

49. Turnbaugh, P.J. and J.I. Gordon, The core gut microbiome, energy balance and obesity. The Journal of physiology, 2009. 587(17): p. 4153-4158.

50. Ley, R.E., Obesity and the human microbiome. Current opinion in gastroenterology, 2010. 26(1): p. 5-11.

51. Yoon, J.C., et al., Peroxisome proliferator-activated receptor $\gamma$ target gene encoding a novel angiopoietin-related protein associated with adipose differentiation. Molecular and cellular biology, 2000. 20(14): p. 5343-5349.

52. Bäckhed, F., et al., The gut microbiota as an environmental factor that regulates fat storage. Proceedings of the National Academy of Sciences of the United States of America, 2004. 101(44): p. 15718-15723. 
53. Musso, G., R. Gambino, and M. Cassader, Interactions between gut microbiota and host metabolism predisposing to obesity and diabetes. Annual review of medicine, 2011. 62: p. 361-380.

54. Ding, S., et al., High-fat diet: bacteria interactions promote intestinal inflammation which precedes and correlates with obesity and insulin resistance in mouse. PloS one, 2010. 5(8): p. e12191.

55. Brinkworth, G.D., et al., Comparative effects of very low-carbohydrate, high-fat and high-carbohydrate, low-fat weight-loss diets on bowel habit and faecal short-chain fatty acids and bacterial populations. British journal of nutrition, 2009. 101(10): p. 14931502.

56. Ley, R.E., et al., Microbial ecology: human gut microbes associated with obesity. Nature, 2006. 444(7122): p. 1022.

57. $\quad \mathrm{Wu}, \mathrm{G} . \mathrm{D}$., et al., Linking long-term dietary patterns with gut microbial enterotypes. Science, 2011. 334(6052): p. 105-108.

58. Serino, M., et al., Metabolic adaptation to a high-fat diet is associated with a change in the gut microbiota. Gut, 2012. 61(4): p. 543-553.

59. Duncan, S.H., et al., Reduced dietary intake of carbohydrates by obese subjects results in decreased concentrations of butyrate and butyrate-producing bacteria in feces. Applied and environmental microbiology, 2007. 73(4): p. 1073-1078.

60. Tilg, H. and A.R. Moschen, Food, immunity, and the microbiome. Gastroenterology, 2015. 148(6): p. 1107-1119.

61. El Kaoutari, A., et al., The abundance and variety of carbohydrate-active enzymes in the human gut microbiota. Nature reviews Microbiology, 2013. 11(7): p. 497-504.

62. Donohoe, D.R., et al., The microbiome and butyrate regulate energy metabolism and autophagy in the mammalian colon. Cell metabolism, 2011. 13(5): p. 517-526.

63. Shapiro, H., et al., The cross talk between microbiota and the immune system: metabolites take center stage. Current opinion in immunology, 2014. 30: p. 54-62.

64. Furusawa, Y., et al., Commensal microbe-derived butyrate induces the differentiation of colonic regulatory T cells. Nature, 2013. 504(7480): p. 446-450.

65. Smith, P.M., et al., The microbial metabolites, short-chain fatty acids, regulate colonic Treg cell homeostasis. Science, 2013. 341(6145): p. 569-573.

66. Samuel, B.S., et al., Effects of the gut microbiota on host adiposity are modulated by the short-chain fatty-acid binding G protein-coupled receptor, Gpr41. Proceedings of the National Academy of Sciences, 2008. 105(43): p. 16767-16772.

67. Collins, M.D. and G.R. Gibson, Probiotics, prebiotics, and synbiotics: approaches for modulating the microbial ecology of the gut. The American Journal of Clinical Nutrition, 1999. 69(5): p. 1052s-1057s.

68. Everard, A., et al., Cross-talk between Akkermansia muciniphila and intestinal epithelium controls diet-induced obesity. Proceedings of the National Academy of Sciences, 2013. 110(22): p. 9066-9071.

69. Plovier, H., et al., A purified membrane protein from Akkermansia muciniphila or the pasteurized bacterium improves metabolism in obese and diabetic mice. Nature Medicine, 2017. 23(1): p. 107-113.

70. Kadooka, Y., et al., Regulation of abdominal adiposity by probiotics (Lactobacillus gasseri SBT2055) in adults with obese tendencies in a randomized controlled trial. European journal of clinical nutrition, 2010. 64(6): p. 636-643. 
71. Cani, P.D., et al., Changes in gut microbiota control inflammation in obese mice through a mechanism involving GLP-2-driven improvement of gut permeability. Gut, 2009. 58(8): p. 1091-1103.

72. Kovatcheva-Datchary, P. and T. Arora, Nutrition, the gut microbiome and the metabolic syndrome. Best Practice \& Research Clinical Gastroenterology, 2013. 27(1): p. 59-72.

73. Ley, R.E., et al., Obesity alters gut microbial ecology. Proceedings of the National Academy of Sciences of the United States of America, 2005. 102(31): p. 11070-11075.

74. Fava, F., et al., The type and quantity of dietary fat and carbohydrate alter faecal microbiome and short-chain fatty acid excretion in a metabolic syndrome 'atrisk'population. International journal of obesity, 2013. 37(2): p. 216-223.

75. Haro, C., et al., The gut microbial community in metabolic syndrome patients is modified by diet. The Journal of nutritional biochemistry, 2016. 27: p. 27-31.

76. Turnbaugh, P.J. and J.I. Gordon, An invitation to the marriage of metagenomics and metabolomics. Cell, 2008. 134(5): p. 708-713.

77. Newgard, C.B., Interplay between lipids and branched-chain amino acids in development of insulin resistance. Cell metabolism, 2012. 15(5): p. 606-614.

78. $\quad$ Rhee, E.P., et al., Lipid profiling identifies a triacylglycerol signature of insulin resistance and improves diabetes prediction in humans. The Journal of clinical investigation, 2011. 121(4): p. 1402-1411.

79. Pflughoeft, K.J. and J. Versalovic, Human microbiome in health and disease. Annual Review of Pathology: Mechanisms of Disease, 2012. 7: p. 99-122.

80. Pedersen, H.K., et al., Human gut microbes impact host serum metabolome and insulin sensitivity. Nature, 2016.

81. Wolf, S., The real gap between bench and bedside. 1974, Mass Medical Soc.

82. Drolet, B.C. and N.M. Lorenzi, Translational research: understanding the continuum from bench to bedside. Translational Research, 2011. 157(1): p. 1-5.

83. Stokols, D., et al., The science of team science: overview of the field and introduction to the supplement. American journal of preventive medicine, 2008. 35(2): p. S77-S89.

84. Huang, T.T.-K., et al., Overweight and components of the metabolic syndrome in college students. Diabetes Care, 2004. 27(12): p. 3000-3001.

85. Morrell, J.S., et al., Metabolic syndrome, obesity, and related risk factors among college men and women. Journal of American College Health, 2012. 60(1): p. 82-89.

86. Das, B.M. and E.M. Evans, Understanding weight management perceptions in first-year college students using the health belief model. Journal of American College Health, 2014. 62(7): p. 488-497.

87. Greaney, M.L., et al., College students' barriers and enablers for healthful weight management: a qualitative study. Journal of nutrition education and behavior, 2009. 41(4): p. 281-286.

88. Huang, T.T.-K., et al., Assessing overweight, obesity, diet, and physical activity in college students. Journal of American College Health, 2003. 52(2): p. 83-86.

89. Kiess, W., et al., Clinical aspects of obesity in childhood and adolescence--diagnosis, treatment and prevention. International Journal of Obesity, 2001. 25(S1): p. S75.

90. McGuire, S., US Department of Agriculture and US Department of Health and Human Services, Dietary Guidelines for Americans, 2010. Washington, DC: US Government Printing Office, January 2011. Advances in Nutrition: An International Review Journal, 2011. 2(3): p. 293-294. 
91. Ebbeling, C.B., et al., Effects of an ad libitum low-glycemic load diet on cardiovascular disease risk factors in obese young adults. The American journal of clinical nutrition, 2005. 81(5): p. 976-982.

92. Noakes, M., et al., Effect of an energy-restricted, high-protein, low-fat diet relative to a conventional high-carbohydrate, low-fat diet on weight loss, body composition, nutritional status, and markers of cardiovascular health in obese women. The American journal of clinical nutrition, 2005. 81(6): p. 1298-1306.

93. Samaha, F.F., et al., A low-carbohydrate as compared with a low-fat diet in severe obesity. New England Journal of Medicine, 2003. 348(21): p. 2074-2081.

94. McMillan-Price, J., et al., Comparison of 4 diets of varying glycemic load on weight loss and cardiovascular risk reduction in overweight and obese young adults: a randomized controlled trial. Archives of internal medicine, 2006. 166(14): p. 1466-1475.

95. Hung, H.-C., et al., Fruit and vegetable intake and risk of major chronic disease. Journal of the National Cancer Institute, 2004. 96(21): p. 1577-1584.

96. Shai, I., et al., Weight loss with a low-carbohydrate, Mediterranean, or low-fat diet. New England Journal of Medicine, 2008. 359(3): p. 229-241.

97. Bowen, D.J. and S.A. Beresford, Dietary interventions to prevent disease. Annual Review of Public Health, 2002. 23(1): p. 255-286.

98. Iversen, A.C. and P. Kraft, Does socio-economic status and health consciousness influence how women respond to health related messages in media? Health education research, 2006. 21(5): p. 601-610.

99. Ha, E.-J. and N. Caine-Bish, Effect of nutrition intervention using a general nutrition course for promoting fruit and vegetable consumption among college students. Journal of Nutrition Education and Behavior, 2009. 41(2): p. 103-109.

100. Lally, P., et al., How are habits formed: Modelling habit formation in the real world. European journal of social psychology, 2010. 40(6): p. 998-1009.

101. Wood, W. and D.T. Neal, A new look at habits and the habit-goal interface. Psychological review, 2007. 114(4): p. 843.

102. Neal, D.T., et al., How do habits guide behavior? Perceived and actual triggers of habits in daily life. Journal of Experimental Social Psychology, 2012. 48(2): p. 492-498.

103. Pelletier, L.G., et al., Why do you regulate what you eat? Relationships between forms of regulation, eating behaviors, sustained dietary behavior change, and psychological adjustment. Motivation and Emotion, 2004. 28(3): p. 245-277.

104. Spahn, J.M., et al., State of the evidence regarding behavior change theories and strategies in nutrition counseling to facilitate health and food behavior change. Journal of the American Dietetic Association, 2010. 110(6): p. 879-891.

105. Cox, W.E., Flight: A Thesis Submitted to the Victoria University of Wellington in Fulfilment of the Requirements for the Degree of Master of Arts in Creative Writing. 2016, Victoria University of Wellington.

106. Hendryx, M. and K.J. Zullig, Higher coronary heart disease and heart attack morbidity in Appalachian coal mining regions. Preventive Medicine, 2009. 49(5): p. 355-359.

107. Schwartz, F., et al., High Self-Reported Prevalence of Diabetes Mellitus, Heart Disease, and Stroke in 11 Counties of Rural Appalachian Ohio. The Journal of Rural Health, 2009. 25(2): p. 226-230. 
108. Halverson, J., L. Ma, and E. Harner, An analysis of disparities in health status and access to health care in the Appalachian region: Executive summary. Washington, DC: Appalachian Regional Commission, 2004.

109. The State of Obesity 2016 - Trust for America's Health. 2017; Available from: http://healthyamericans.org/reports/stateofobesity2016/.

110. Li, C., et al., Surveillance of certain health behaviors and conditions among states and selected local areas-Behavioral Risk Factor Surveillance System, United States, 2009. Morbidity and Mortality Weekly Report: Surveillance Summaries, 2011. 60(9): p. 1-249.

111. Branen, L. and J. Fletcher, Comparison of college students' current eating habits and recollections of their childhood food practices. Journal of Nutrition Education, 1999. 31(6): p. 304-310.

112. Ben-Noun, L.L. and A. Laor, Relationship between changes in neck circumference and cardiovascular risk factors. Experimental \& Clinical Cardiology, 2006. 11(1): p. 14.

113. Wallace, T.M., J.C. Levy, and D.R. Matthews, Use and abuse of HOMA modeling. Diabetes care, 2004. 27(6): p. 1487-1495.

114. Freedson, P.S., E. Melanson, and J. Sirard, Calibration of the Computer Science and Applications, Inc. accelerometer. Medicine and science in sports and exercise, 1998. 30(5): p. 777-781.

115. Buysse, D.J., et al., The Pittsburgh Sleep Quality Index: a new instrument for psychiatric practice and research. Psychiatry research, 1989. 28(2): p. 193-213.

116. Cohen, S., T. Kamarck, and R. Mermelstein, Perceived stress scale. Measuring stress: A guide for health and social scientists, 1994.

117. Kristal, A.R., et al., A randomized trial of a tailored, self-help dietary intervention: the Puget Sound Eating Patterns study. Preventive medicine, 2000. 31(4): p. 380-389.

118. Brinberg, D., M.L. Axelson, and S. Price, Changing food knowledge, food choice, and dietary fiber consumption by using tailored messages. Appetite, 2000. 35(1): p. 35-43.

119. Chobanian, A.V., et al., The seventh report of the joint national committee on prevention, detection, evaluation, and treatment of high blood pressure: the JNC 7 report. Jama, 2003. 289(19): p. 2560-2571.

120. De Koning, L., et al., Waist circumference and waist-to-hip ratio as predictors of cardiovascular events: meta-regression analysis of prospective studies. European heart journal, 2007. 28(7): p. 850-856.

121. Law, M., J. Morris, and N. Wald, Use of blood pressure lowering drugs in the prevention of cardiovascular disease: meta-analysis of 147 randomised trials in the context of expectations from prospective epidemiological studies. Bmj, 2009. 338: p. b1665.

122. Whelton, P.K., et al., Effects of oral potassium on blood pressure: meta-analysis of randomized controlled clinical trials. Jama, 1997. 277(20): p. 1624-1632.

123. Klein, S., et al., Association for Weight Management and Obesity Prevention; NAASO, The Obesity Society; American Society for Nutrition; American Diabetes Association. Waist circumference and cardiometabolic risk: a consensus statement from Shaping America \'s Health: Association for Weight Management and Obesity Prevention; NAASO, The Obesity Society; the American Society for Nutrition; and the American Diabetes Association. Am J Clin Nutr, 2007. 85: p. 1197-1202.

124. Preis, S.R., et al., Neck circumference as a novel measure of cardiometabolic risk: the Framingham Heart study. The journal of clinical endocrinology \& metabolism, 2010. 95(8): p. 3701-3710. 
125. Wing, R.R., et al., Benefits of modest weight loss in improving cardiovascular risk factors in overweight and obese individuals with type 2 diabetes. Diabetes care, 2011. 34(7): p. 1481-1486.

126. Singh, I.M., M.H. Shishehbor, and B.J. Ansell, High-density lipoprotein as a therapeutic target: a systematic review. Jama, 2007. 298(7): p. 786-798.

127. Jenkins, D., et al., Effect on blood lipids of very high intakes of fiber in diets low in saturated fat and cholesterol. New England Journal of Medicine, 1993. 329(1): p. 21-26.

128. Turley, M., et al., The effect of a low-fat, high-carbohydrate diet on serum high density lipoprotein cholesterol and triglyceride. European journal of clinical nutrition, 1998. 52(10): p. 728-732.

129. Lemieux, I., et al., Total cholesterol/HDL cholesterol ratio vs LDL cholesterol/HDL cholesterol ratio as indices of ischemic heart disease risk in men: the Quebec Cardiovascular Study. Archives of internal medicine, 2001. 161(22): p. 2685-2692.

130. Manninen, V., et al., Joint effects of serum triglyceride and LDL cholesterol and HDL cholesterol concentrations on coronary heart disease risk in the Helsinki Heart Study. Implications for treatment. Circulation, 1992. 85(1): p. 37-45.

131. Mensink, R.P., et al., Effects of dietary fatty acids and carbohydrates on the ratio of serum total to HDL cholesterol and on serum lipids and apolipoproteins: a meta-analysis of 60 controlled trials. The American journal of clinical nutrition, 2003. 77(5): p. 11461155.

132. Müller, H., et al., The serum LDL/HDL cholesterol ratio is influenced more favorably by exchanging saturated with unsaturated fat than by reducing saturated fat in the diet of women. The Journal of nutrition, 2003. 133(1): p. 78-83.

133. Law, M.R., N.J. Wald, and S. Thompson, By how much and how quickly does reduction in serum cholesterol concentration lower risk of ischaemic heart disease? Bmj, 1994. 308(6925): p. 367-372.

134. Siero, F., et al., Impact of group nutrition education and surplus value of Prochaskabased stage-matched information on health-related cognitions and on Mediterranean nutrition behavior. Health Education Research, 2000. 15(5): p. 635-647.

135. Ornish, D., et al., Intensive lifestyle changes for reversal of coronary heart disease. Jama, 1998. 280(23): p. 2001-2007.

136. Laska, M.N., et al., Results of a 2-year randomized, controlled obesity prevention trial: Effects on diet, activity and sleep behaviors in an at-risk young adult population.

Preventive medicine, 2016. 89: p. 230-236.

137. Cleeman, J.I. and S.M. Grundy, National Cholesterol Education Program recommendations for cholesterol testing in young adults. Circulation, 1997. 95(6): p. 1646-1650.

138. Gerhard, G.T., et al., Effects of a low-fat diet compared with those of a highmonounsaturated fat diet on body weight, plasma lipids and lipoproteins, and glycemic control in type 2 diabetes. The American journal of clinical nutrition, 2004. 80(3): p. 668673.

139. Spring, B., et al., Multiple behavior changes in diet and activity: a randomized controlled trial using mobile technology. Archives of internal medicine, 2012. 172(10): p. 789-796.

140. Statistics, N.C.f.H., Plan and operation of the third National Health and Nutrition Examination Survey, 1988-94. 1994: Natl Ctr for Health Statistics. 
141. Yusuf, S., et al., Effect of potentially modifiable risk factors associated with myocardial infarction in 52 countries (the INTERHEART study): case-control study. The lancet, 2004. 364(9438): p. 937-952.

142. Keller, I. and C. Tukuitonga. The WHO/FAO fruit and vegetable promotion initiative. in I International Symposium on Human Health Effects of Fruits and Vegetables 744. 2005.

143. Esposito, K., et al., Effects of a Mediterranean-Style Diet on the Need for Antihyperglycemic Drug Therapy in Patients With Newly Diagnosed Type 2 DiabetesA Randomized Trial. Annals of internal medicine, 2009. 151(5): p. 306-314.

144. Elhayany, A., et al., A low carbohydrate Mediterranean diet improves cardiovascular risk factors and diabetes control among overweight patients with type 2 diabetes mellitus: a 1-year prospective randomized intervention study. Diabetes, Obesity and Metabolism, 2010. 12(3): p. 204-209.

145. Estruch, R., et al., Primary prevention of cardiovascular disease with a Mediterranean diet. New England Journal of Medicine, 2013. 368(14): p. 1279-1290.

146. Murphy, E., et al., Composition and energy harvesting capacity of the gut microbiota: relationship to diet, obesity and time in mouse models. Gut, 2010. 59(12): p. 1635-1642.

147. Turnbaugh, P.J., et al., An obesity-associated gut microbiome with increased capacity for energy harvest. nature, 2006. 444(7122): p. 1027-131.

148. Schwiertz, A., et al., Microbiota and SCFA in lean and overweight healthy subjects. Obesity, 2010. 18(1): p. 190-195.

149. Nordin, S.A., et al., Metagenome: Differences in the Gut Microbiota among Healthy, Obese and Type 2 Diabetes Adults. Genomics and Applied Biology, 2016. 7(3).

150. Zhang, C., et al., Interactions between gut microbiota, host genetics and diet relevant to development of metabolic syndromes in mice. The ISME journal, 2010. 4(2): p. 232-241.

151. Holmes, E., et al., Gut microbiota composition and activity in relation to host metabolic phenotype and disease risk. Cell metabolism, 2012. 16(5): p. 559-564.

152. Cox, L.M., et al., Altering the intestinal microbiota during a critical developmental window has lasting metabolic consequences. Cell, 2014. 158(4): p. 705-721.

153. Koeth, R.A., et al., Intestinal microbiota metabolism of L-carnitine, a nutrient in red meat, promotes atherosclerosis. Nature medicine, 2013. 19(5): p. 576-585.

154. Kotronen, A., et al., Serum saturated fatty acids containing triacylglycerols are better markers of insulin resistance than total serum triacylglycerol concentrations. Diabetologia, 2009. 52(4): p. 684.

155. Floegel, A., et al., Identification of serum metabolites associated with risk of type 2 diabetes using a targeted metabolomic approach. Diabetes, 2013. 62(2): p. 639-648.

156. Lee, C.C., et al., Branched-chain amino acids and insulin metabolism: the Insulin Resistance Atherosclerosis Study (IRAS). Diabetes Care, 2016. 39(4): p. 582-588.

157. Morrell, J.S., et al., Metabolic Syndrome: Comparison of Prevalence in Young Adults at 3 Land-Grant Universities. Journal of American College Health, 2013. 62(1): p. 109.

158. Peterkin, B., Dietary guidelines for Americans. Journal of the American Dietetic Association, 1990. 90(12): p. 1725-1727.

159. Elmore, J.S., et al., The effect of cooking on acrylamide and its precursors in potato, wheat and rye, in Chemistry and safety of acrylamide in food. 2005, Springer. p. 255-269.

160. Badawy, A.-B., C. Morgan, and J. Turner, Application of the Phenomenex EZ: faast $\mathrm{TM}^{\mathrm{TM}}$ amino acid analysis kit for rapid gas-chromatographic determination of concentrations 
of plasma tryptophan and its brain uptake competitors. Amino acids, 2008. 34(4): p. 587596.

161. Flores, G.E., J.B. Henley, and N. Fierer, A direct PCR approach to accelerate analyses of human-associated microbial communities. PloS one, 2012. 7(9): p. e44563.

162. Amplicon, P., P. Clean-Up, and P. Index, 16S Metagenomic Sequencing Library Preparation.

163. Caporaso, J.G., et al., QIIME allows analysis of high-throughput community sequencing data. Nature methods, 2010. 7(5): p. 335-336.

164. Pham, T.V. and C.R. Jimenez, An accurate paired sample test for count data. Bioinformatics, 2012. 28(18): p. i596-i602.

165. Langille, M.G., et al., Predictive functional profiling of microbial communities using $16 \mathrm{~S}$ rRNA marker gene sequences. Nature biotechnology, 2013. 31(9): p. 814-821.

166. Kanehisa, M. and S. Goto, KEGG: kyoto encyclopedia of genes and genomes. Nucleic acids research, 2000. 28(1): p. 27-30.

167. Turnbaugh, P.J., et al., The effect of diet on the human gut microbiome: a metagenomic analysis in humanized gnotobiotic mice. Science translational medicine, 2009. 1(6): p. 6ra14-6ra14.

168. Kissebah, A.H., et al., Relation of body fat distribution to metabolic complications of obesity. The Journal of Clinical Endocrinology \& Metabolism, 1982. 54(2): p. 254-260.

169. A. Parnell, J. and R. A. Reimer, Prebiotic fiber modulation of the gut microbiota improves risk factors for obesity and the metabolic syndrome. Gut microbes, 2012. 3(1): p. 29-34.

170. De Filippis, F., et al., High-level adherence to a Mediterranean diet beneficially impacts the gut microbiota and associated metabolome. Gut, 2015: p. gutjnl-2015-309957.

171. Ishiguro, K., et al., Suppressive action of acetate on interleukin-8 production via tubulin$\alpha$ acetylation. Immunology and cell biology, 2014. 92(7): p. 624-630.

172. Lin, H.V., et al., Butyrate and propionate protect against diet-induced obesity and regulate gut hormones via free fatty acid receptor 3-independent mechanisms. PloS one, 2012. 7(4): p. e35240.

173. Batterham, R.L., et al., Gut hormone PYY3-36 physiologically inhibits food intake. Nature, 2002. 418(6898): p. 650-654.

174. Lange, K., Molecular mechanisms underlying the effects of dietary fiber in the large intestine. 2015: Wageningen University.

175. Krych, Ł., et al., Gut microbial markers are associated with diabetes onset, regulatory imbalance, and IFN- $\gamma$ level in NOD Mice. Gut microbes, 2015. 6(2): p. 101-109.

176. Liou, A.P., et al., Conserved shifts in the gut microbiota due to gastric bypass reduce host weight and adiposity. Science translational medicine, 2013. 5(178): p. 178ra41178 ra41.

177. Salyers, A., et al., Fermentation of mucin and plant polysaccharides by strains of Bacteroides from the human colon. Applied and environmental microbiology, 1977. 33(2): p. 319-322.

178. Wrzosek, L., et al., Bacteroides thetaiotaomicron and Faecalibacterium prausnitzii influence the production of mucus glycans and the development of goblet cells in the colonic epithelium of a gnotobiotic model rodent. BMC biology, 2013. 11(1): p. 61.

179. Gill, S.R., et al., Metagenomic analysis of the human distal gut microbiome. science, 2006. 312(5778): p. 1355-1359. 
180. Elsden, S.R., M.G. Hilton, and J.M. Waller, The end products of the metabolism of aromatic amino acids by Clostridia. Archives of Microbiology, 1976. 107(3): p. 283-288.

181. Lin, H., et al., Correlations of fecal metabonomic and microbiomic changes induced by high-fat diet in the pre-obesity state. Scientific reports, 2016. 6.

182. Costabile, A., et al., Whole-grain wheat breakfast cereal has a prebiotic effect on the human gut microbiota: a double-blind, placebo-controlled, crossover study. British Journal of Nutrition, 2008. 99(01): p. 110-120.

183. Ford, E.S. and A.H. Mokdad, Fruit and vegetable consumption and diabetes mellitus incidence among US adults. Preventive medicine, 2001. 32(1): p. 33-39.

184. Field, A.E., et al., Association between fruit and vegetable intake and change in body mass index among a large sample of children and adolescents in the United States. International journal of obesity, 2003. 27(7): p. 821-826.

185. Serdula, M.K., et al., The association between fruit and vegetable intake and chronic disease risk factors. Epidemiology, 1996. 7(2): p. 161-165.

186. Williams, D.E., et al., Frequent salad vegetable consumption is associated with a reduction in the risk of diabetes mellitus. Journal of clinical epidemiology, 1999. 52(4): p. 329-335.

187. Lin, B.-H. and R.M. Morrison, Higher fruit consumption linked with lower body mass index. Food review, 2002. 25(3): p. 28-32.

188. Joshipura, K.J., et al., The effect of fruit and vegetable intake on risk for coronary heart disease. Annals of internal medicine, 2001. 134(12): p. 1106-1114.

189. Wang, Y., et al., Comparison of abdominal adiposity and overall obesity in predicting risk of type 2 diabetes among men. The American journal of clinical nutrition, 2005. 81(3): p. 555-563.

190. Salas-Salvadó, J., et al., The role of diet in the prevention of type 2 diabetes. Nutrition, Metabolism and Cardiovascular Diseases, 2011. 21: p. B32-B48.

191. Holland, W.L. and S.A. Summers, Sphingolipids, insulin resistance, and metabolic disease: new insights from in vivo manipulation of sphingolipid metabolism. Endocrine reviews, 2008. 29(4): p. 381-402.

192. Boon, J., et al., Ceramides contained in LDL are elevated in type 2 diabetes and promote inflammation and skeletal muscle insulin resistance. Diabetes, 2013. 62(2): p. 401-410.

193. Bikman, B.T. and S.A. Summers, Sphingolipids and hepatic steatosis, in Sphingolipids and Metabolic Disease. 2011, Springer. p. 87-97.

194. Chavez, J.A., et al., Ceramides and glucosylceramides are independent antagonists of insulin signaling. Journal of Biological Chemistry, 2014. 289(2): p. 723-734.

195. Lopez, X., et al., Plasma ceramides are elevated in female children and adolescents with type 2 diabetes. Journal of Pediatric Endocrinology and Metabolism, 2013 . 26(9-10): p. 995-998.

196. Meikle, P.J., et al., Plasma lipid profiling shows similar associations with prediabetes and type 2 diabetes. PloS one, 2013. 8(9): p. e74341.

197. Summers, S.A., Ceramides in insulin resistance and lipotoxicity. Progress in lipid research, 2006. 45(1): p. 42-72.

198. Fabbrini, E., S. Sullivan, and S. Klein, Obesity and nonalcoholic fatty liver disease: biochemical, metabolic, and clinical implications. Hepatology, 2010. 51(2): p. 679-689. 
199. Vila, L., et al., Suppressor of cytokine signaling-3 (SOCS-3) and a deficit of serine/threonine (Ser/Thr) phosphoproteins involved in leptin transduction mediate the effect of fructose on rat liver lipid metabolism. Hepatology, 2008. 48(5): p. 1506-1516.

200. Assy, N., et al., Soft drink consumption linked with fatty liver in the absence of traditional risk factors. Canadian Journal of Gastroenterology and Hepatology, 2008. 22(10): p. 811816.

201. Asrih, M. and F.R. Jornayvaz, Diets and nonalcoholic fatty liver disease: the good and the bad. Clinical Nutrition, 2014. 33(2): p. 186-190.

202. Hla, T. and R. Kolesnick, C16: O-ceramide signals insulin resistance. Cell metabolism, 2014. 20(5): p. 703-705.

203. Raichur, S., et al., CerS2 haploinsufficiency inhibits $\beta$-oxidation and confers susceptibility to diet-induced steatohepatitis and insulin resistance. Cell metabolism, 2014. 20(4): p. 687-695.

204. Turpin, S.M., et al., Obesity-induced CerS6-dependent C 16: 0 ceramide production promotes weight gain and glucose intolerance. Cell metabolism, 2014. 20(4): p. 678-686.

205. Krauss, R.M., et al., AHA scientific statement: AHA dietary guidelines Revision 2000: A statement for healthcare professionals from the nutrition committee of the american heart Association. The Journal of nutrition, 2001. 131(1): p. 132-146.

206. Haughey, N.J., et al., Perturbation of sphingolipid metabolism and ceramide production in HIV-dementia. Annals of neurology, 2004. 55(2): p. 257-267.

207. Rico, J., et al., Plasma ceramides are elevated in overweight Holstein dairy cows experiencing greater lipolysis and insulin resistance during the transition from late pregnancy to early lactation. Journal of dairy science, 2015. 98(11): p. 7757-7770.

208. Mielke, M.M., et al., Demographic and clinical variables affecting mid-to late-life trajectories of plasma ceramide and dihydroceramide species. Aging cell, 2015. 14(6): p. 1014-1023.

209. Srinivasan, S.R., et al., Adolescent overweight is associated with adult overweight and related multiple cardiovascular risk factors: the Bogalusa Heart Study. Metabolism, 1996. 45(2): p. 235-240.

210. Rader, D.J. and G.K. Hovingh, HDL and cardiovascular disease. The Lancet, 2014. 384(9943): p. 618-625.

211. Holland, W.L., et al., Inhibition of ceramide synthesis ameliorates glucocorticoid-, saturated-fat-, and obesity-induced insulin resistance. Cell metabolism, 2007. 5(3): p. 167-179.

212. Brozinick, J., et al., Plasma sphingolipids are biomarkers of metabolic syndrome in nonhuman primates maintained on a Western-style diet. International journal of obesity, 2013. 37(8): p. 1064-1070.

213. Haus, J.M., et al., Plasma ceramides are elevated in obese subjects with type 2 diabetes and correlate with the severity of insulin resistance. Diabetes, 2009. 58(2): p. 337-343.

214. Holland, W.L., et al., Lipid-induced insulin resistance mediated by the proinflammatory receptor TLR4 requires saturated fatty acid-induced ceramide biosynthesis in mice. The Journal of clinical investigation, 2011. 121(5): p. 1858-1870.

215. Kasumov, T., et al., Ceramide as a mediator of non-alcoholic Fatty liver disease and associated atherosclerosis. PloS one, 2015. 10(5): p. e0126910. 
216. Dube, J., et al., Effects of weight loss and exercise on insulin resistance, and intramyocellular triacylglycerol, diacylglycerol and ceramide. Diabetologia, 2011. 54(5): p. 1147-1156.

217. Dekker, M.J., et al., Inhibition of sphingolipid synthesis improves dyslipidemia in the diet-induced hamster model of insulin resistance: evidence for the role of sphingosine and sphinganine in hepatic VLDL-apoB100 overproduction. Atherosclerosis, 2013. 228(1): p. 98-109.

218. Lightle, S., et al., Elevation of ceramide in serum lipoproteins during acute phase response in humans and mice: role of serine-palmitoyl transferase. Archives of biochemistry and biophysics, 2003. 419(2): p. 120-128.

219. Laviad, E.L., et al., Characterization of Ceramide Synthase 2 Tissue distribution, substrate specificity, and inhibition by sphingosine 1-phosphate. Journal of Biological Chemistry, 2008. 283(9): p. 5677-5684.

220. Larsen, P.J. and N. Tennagels, On ceramides, other sphingolipids and impaired glucose homeostasis. Molecular metabolism, 2014. 3(3): p. 252-260.

221. Goñi, F.M. and A. Alonso, Biophysics of sphingolipids I. Membrane properties of sphingosine, ceramides and other simple sphingolipids. Biochimica et Biophysica Acta (BBA)-Biomembranes, 2006. 1758(12): p. 1902-1921.

222. Aerts, J.M., et al., Pharmacological inhibition of glucosylceramide synthase enhances insulin sensitivity. Diabetes, 2007. 56(5): p. 1341-1349.

223. Vainio, S., et al., Dynamic association of human insulin receptor with lipid rafts in cells lacking caveolae. EMBO reports, 2002. 3(1): p. 95-100.

224. Kabayama, K., et al., TNFa-induced insulin resistance in adipocytes as a membrane microdomain disorder: involvement of ganglioside GM3. Glycobiology, 2005. 15(1): p. 21-29.

225. Barber, M.N., et al., Plasma lysophosphatidylcholine levels are reduced in obesity and type 2 diabetes. PloS one, 2012. 7(7): p. e41456.

226. Dandona, P., A. Aljada, and A. Bandyopadhyay, Inflammation: the link between insulin resistance, obesity and diabetes. Trends in immunology, 2004. 25(1): p. 4-7.

227. Esposito, K., et al., Effect of weight loss and lifestyle changes on vascular inflammatory markers in obese women: a randomized trial. Jama, 2003. 289(14): p. 1799-1804.

228. García-Lafuente, A., et al., Flavonoids as anti-inflammatory agents: implications in cancer and cardiovascular disease. Inflammation Research, 2009. 58(9): p. 537-552.

229. Holt, E.M., et al., Fruit and vegetable consumption and its relation to markers of inflammation and oxidative stress in adolescents. Journal of the American Dietetic Association, 2009. 109(3): p. 414-421.

230. Food and D. Administration, Guidance for industry: patient-reported outcome measures: use in medical product development to support labeling claims. Fed Regist, 2009. 74(235): p. 65132-65133.

231. Galesic, M. and M. Bosnjak, Effects of questionnaire length on participation and indicators of response quality in a web survey. Public opinion quarterly, 2009. 73(2): p. 349-360.

232. Krosnick, J.A., et al., The impact of" no opinion" response options on data quality: nonattitude reduction or an invitation to satisfice? Public Opinion Quarterly, 2002. 66(3): p. 371-403. 
233. Herzog, A.R. and J.G. Bachman, Effects of questionnaire length on response quality. Public opinion quarterly, 1981. 45(4): p. 549-559.

234. Ware Jr, J.E., M. Kosinski, and S.D. Keller, A 12-Item Short-Form Health Survey: construction of scales and preliminary tests of reliability and validity. Medical care, 1996. 34(3): p. 220-233.

235. Bize, R. and R.C. Plotnikoff, The relationship between a short measure of health status and physical activity in a workplace population. Psychology, health \& medicine, 2009. 14(1): p. 53-61.

236. Pilcher, J.J., D.R. Ginter, and B. Sadowsky, Sleep quality versus sleep quantity: relationships between sleep and measures of health, well-being and sleepiness in college students. Journal of psychosomatic research, 1997. 42(6): p. 583-596.

237. St-Onge, M.-P., et al., Short sleep duration increases energy intakes but does not change energy expenditure in normal-weight individuals. The American journal of clinical nutrition, 2011. 94(2): p. 410-416.

238. Forquer, L.M., et al., Sleep patterns of college students at a public university. Journal of American College Health, 2008. 56(5): p. 563-565.

239. Buboltz Jr, W.C., F. Brown, and B. Soper, Sleep habits and patterns of college students: a preliminary study. Journal of American college health, 2001. 50(3): p. 131-135.

240. Bornstein, S.K., Respiratory monitoring during sleep: polysomnography. Sleeping and Waking Disorders: Indications and Techniques. Addison-Wesley, Menlo Park, CA pp, 1982: p. 183-212.

241. Block, G., et al., A rapid food screener to assess fat and fruit and vegetable intake. American journal of preventive medicine, 2000. 18(4): p. 284-288.

242. Booth, M.L., et al., International physical activity questionnaire: 12-country reliability and validity. Med sci sports Exerc, 2003. 195(9131/03): p. 3508-1381. 


\section{Vita}

Oluremi A Famodu received a B.S. degree in Nutritional Sciences from Ohio University in Athens, Ohio. She then received her M.S. degree in Human Nutrition and Food Sciences at West Virginia University under the advisement of Dr. Melissa Olfert. Her research focused on the effect of sleep extension of female athlete's physical and mental performance, as well as dietary behaviors. Along with obtaining her M.S. degree, she was a dietetic intern under Dr. Melissa Olfert and completed the requirements to obtain her Registered Dietitian Nutritionist certification. For the past 3 years, Oluremi has been working on her Ph.D. degree in Animal and Nutritional Sciences at WVU, in the Lifestyle Intervention Research Lab under Dr. Melissa Olfert, where her work has focused on the impact of a healthy diet interventions on colonic bacteria composition in young adults. 